\title{
A PREDICTION OF THE ACOUSTICAL OUTPUT OF A GOLF DRIVER HEAD USING
} FINITE ELEMENTS

\author{
A Thesis \\ presented to \\ the Faculty of California Polytechnic State University, \\ San Luis Obispo
}

\author{
In Partial Fulfillment \\ of the Requirements for the Degree \\ Master of Science in Mechanical Engineering \\ by \\ Roger Sharpe
}

March 2010 
(C) 2010

Roger Sharpe

ALL RIGHTS RESERVED 


\section{COMMITTEE MEMBERSHIP}

TITLE:

A PREDICTION OF THE ACOUSTICAL OUTPUT OF A GOLF DRIVER HEAD USING FINITE ELEMENTS

AUTHOR: $\quad$ Roger Sharpe

DATE SUBMITTED: November 2009

COMMITTEE CHAIR: Dr. Tom Mase

COMMITTEE MEMBER: Dr. James Meagher

COMMITTEE MEMBER: Dr. Peter Schuster 


\begin{abstract}
A PREDICTION OF THE ACOUSTICAL OUTPUT OF A GOLF DRIVER HEAD USING FINITE ELEMENTS

Roger Sharpe
\end{abstract}

\begin{abstract}
A simulation was created using LS-DYNA ${ }^{\circledR}$ to determine the acoustical properties of a golf ball and golf driver head impact. LS-DYNA® has a coupled finite element analysis (FEA) and boundary element method (BEM) solver that uses the integral form of Helmholtz's acoustic wave equation to deliver predicted sound pressure levels at predetermined acoustic points. Validation of the modeling was done on a simple plate donated by Titleist Golf. The plate was modeled and meshed using TrueGrid ${ }^{\circledR}$ and impacted by a three layer golf ball model derived from “Tanka's" paper on multilayered golf balls. The final converging model consisted of 10,900 solid fully integrated elements between the ball, plate, and plate support structure. The result was compared to experimental data taken by an air cannon and anechoic chamber that housed strain and acoustical measurement equipment. The sound level predictions from the model showed a promising correlation with experimental data and the focus switched to a golf driver head response during impact.
\end{abstract}

The same ball developed from Tanaka's paper was used to impact a 350cc generic golf driver head. The driver head consisted of 3300 fully integrated shell elements throughout the model. The top of the hosel was fixed during the simulation to simulate the connection to the golf shaft. The ball was fired at the center of the driver's face and the predicted sound was determined for a point two feet behind the driver head. The BEM prediction of the driver head model showed little correlation with actual recorded impact sounds provided by Cleveland Golf when comparing frequency response functions. These differences could arise from assumptions and iv $\mid \mathrm{P}$ a g e 
simplifications made to speed up the impact simulation. The sound produced from the golf ball after impact was one such factor was not included. Due to the complex shape of the driver head and the total number of elements involved, the numerical solution took upwards of 100 hours to finish. Adding the golf ball sound would greatly increase computational time and not contribute significantly to the overall predicted sound. Although the BEM solution can be used to characterize different driver heads, the impact is too complicated to efficiently and accurately predict the true impact sounds. 


\section{TABLE OF CONTENTS}

\section{Page}

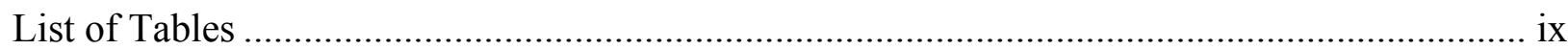

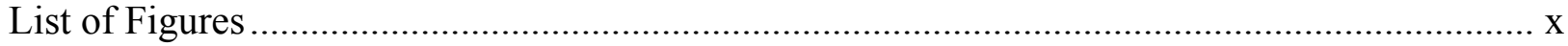

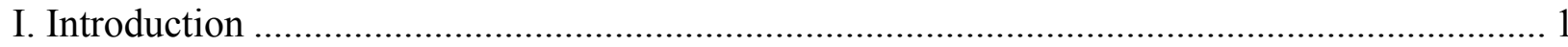

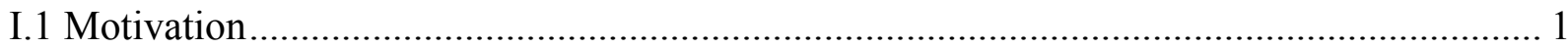

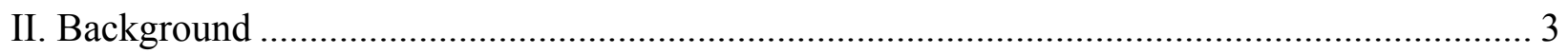

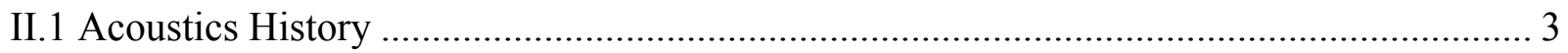

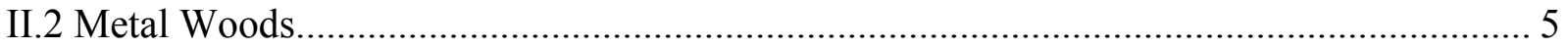

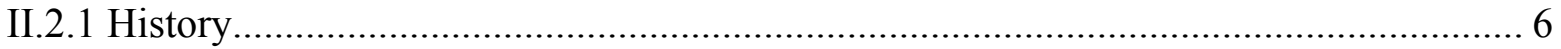

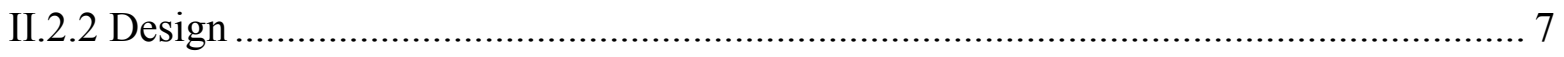

II.2.3 Sound Production....................................................................................... 8

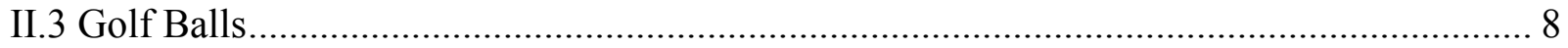

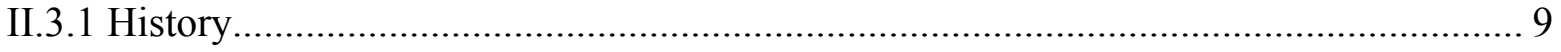

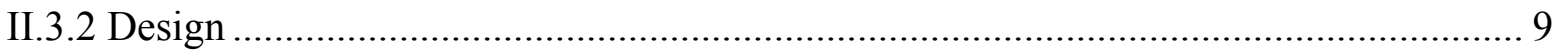

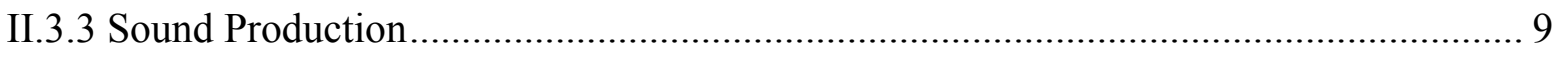

II.4 Methods to Determine Sound Performance.............................................................. 10

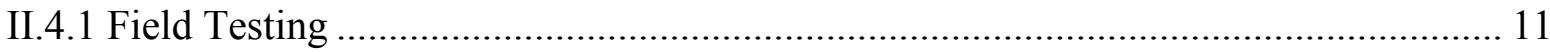

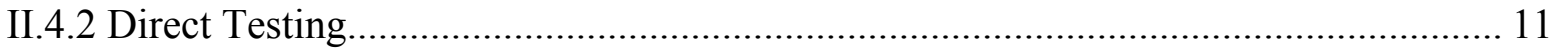

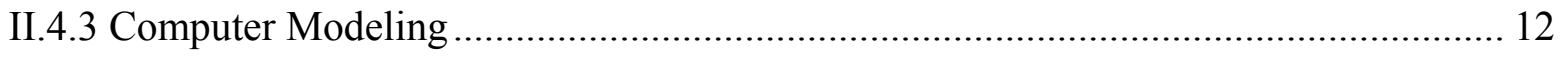




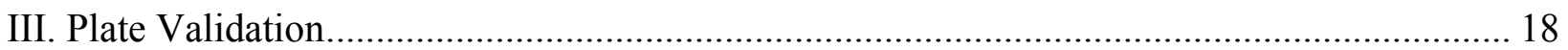

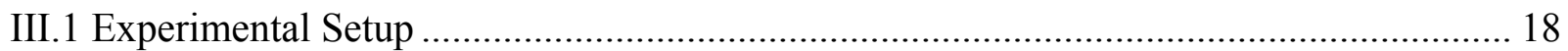

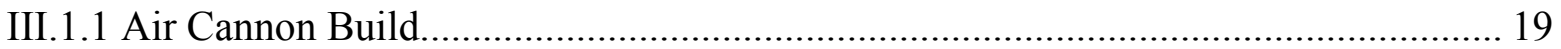

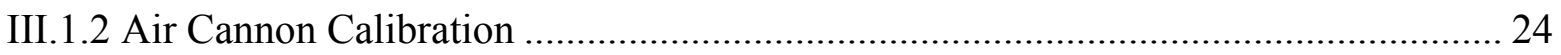

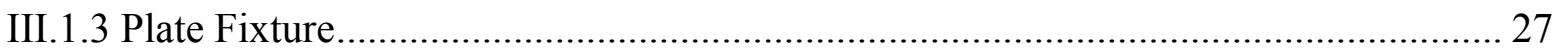

III.2 Computer Simulation of the Titanium Plate ………...................................................... 30

III.2.1 Computer Modeling of Plate …………………………………………………..... 31

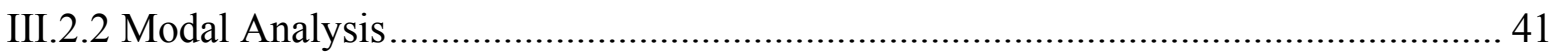

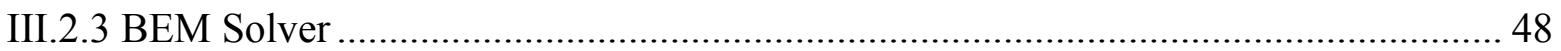

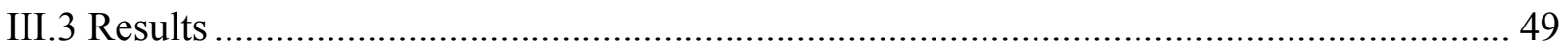

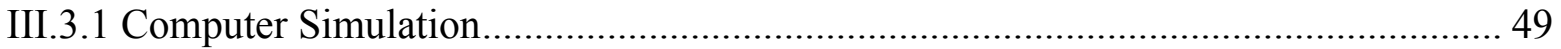

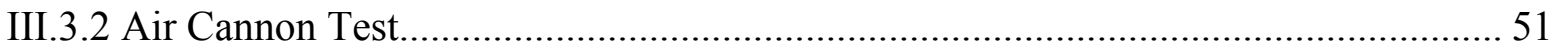

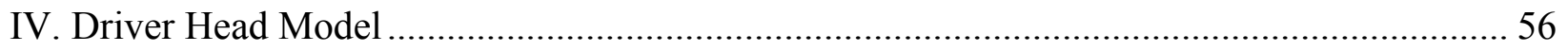

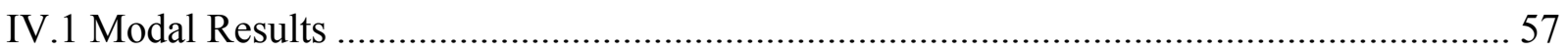

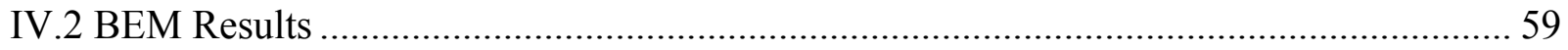

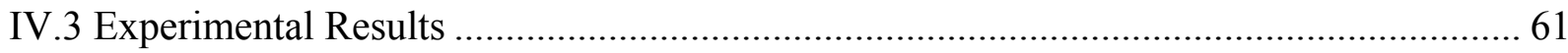

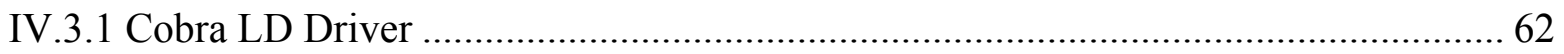

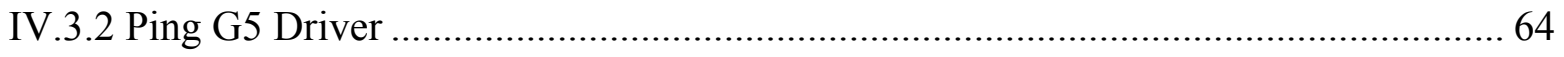

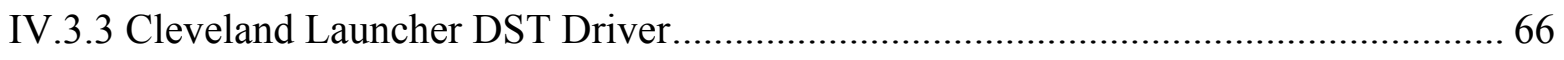

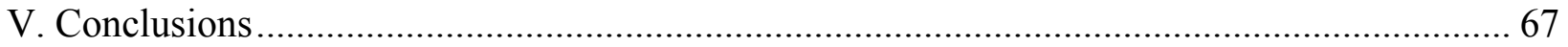


References.

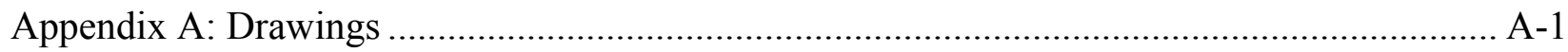

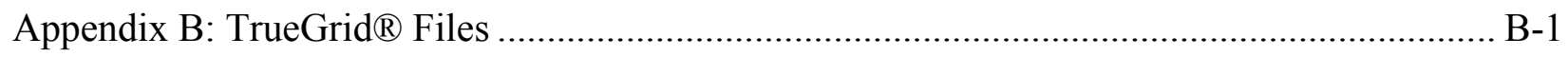

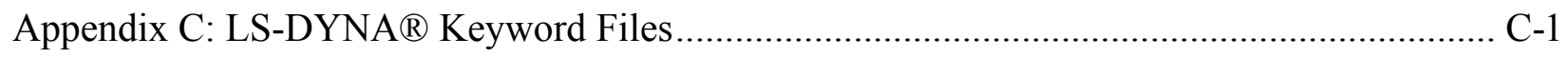

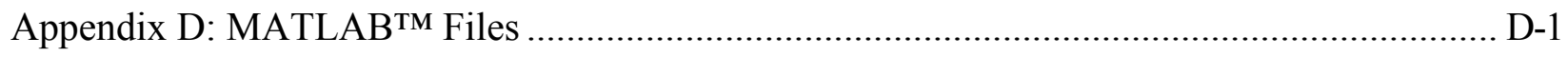




\section{LIST OF TABLES}

Table 1: Common metals used in driver head construction and their properties from

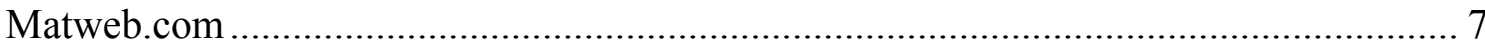

Table 2: Mesh convergence data from the ball and plate. .................................................... 35

Table 3: Golf ball material properties provided by Tanaka................................................... 38

Table 4: Derived coefficients for shear moduli $G_{i}$ and decay constants $\beta_{i}$ in the Prony series

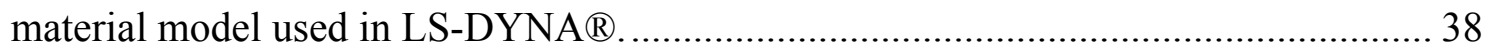

Table 5: Curve fitting coefficients used in the Prony series................................................. 39

Table 6: Mode shapes derived by Blevin.................................................................... 42

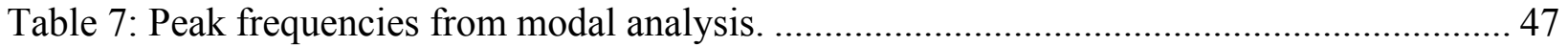




\section{LIST OF FIGURES}

Figure 1: Definition of the problem, determining sound of an arbitrary object at a point in space

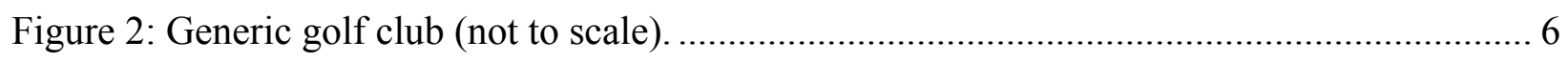

Figure 3: Laser holography picture of the sound produced from a Callaway Big Bertha driver. 11

Figure 4: Flow of programs used to create simulated impact sound. ................................... 13

Figure 5: Flow of operations performed by LS-DYNA® in the coupled FEA/BEM solver........ 15

Figure 6: Boundary element card variable description from LSTC press release. .................... 16

Figure 7: Experimental setup to determine the sound output of the titanium plate. Shown is the air cannon, plate support structure and data acquisition system. ............................ 19

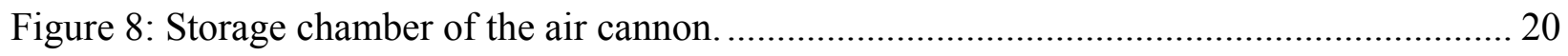

Figure 9: Air pressure sensor connected to the storage chamber.......................................... 22

Figure 10: Solenoid (a) cross-section from McMaster and (b) in use. .................................. 23

Figure 11: Air cannon placed on test stand behind 1/2" polycarbonate. ................................. 24

Figure 12: Phantom v310 high speed camera from visionresearch.com ............................... 25

Figure 13: Calibration curve for the air cannon.............................................................. 26

Figure 14: Titleist ProV1 golf ball exiting the air cannon with little to no spin....................... 27

Figure 15: Titanium plate support structure using T-slot extrusions .................................... 28

Figure 16: Titanium plate secured into the plate fixture using mounting brackets. ................... 29

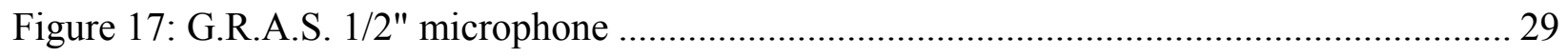

Figure 18: Inputs plugged into the back of the LDS signal analyzer..................................... 30

Figure 19: Cross-section of the titanium plate highlighting how the thickness changes............ 31

Figure 20: Load curve used for mesh convergence. ....................................................... 32 
Figure 21: Boundary conditions of mesh convergence. 32

Figure 22: Mesh convergence for the plate 33

Figure 23: Setup for ball and plate mesh convergence with fixed boundary conditions. 34

Figure 24: Mesh convergence for the ball and plate. 34

Figure 25: SolidWorks rendering of the machined support brackets for the titanium plate......... 36

Figure 26: Titleist ProV1 golf ball cut in half showing the core, mantle, and cover thicknesses.

Figure 27: Tanaka's force and contact time plot (a) compared to the FE model (b) used for golf ball mesh convergence. 39

Figure 28: Cross section of the meshed golf ball showing the different materials used for the core, mantle, and cover using mesh density No. 5 . 40

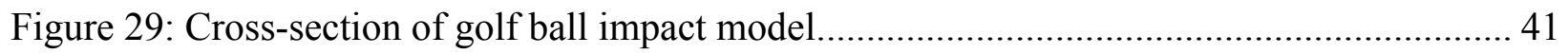

Figure 30: Titanium plate cut along the diameter showing the change in thickness. ................... 41

Figure 31: FFT plot of acceleration from the titanium plate excited in a free condition using a force hammer.

Figure 32: FEA result of the first modal frequency with a nodal circle.

Figure 33: FEA results of the second modal frequency with two nodal circles. .......................... 45

Figure 34: FFT plot of acceleration of the center plate and sound pressure ............................... 46

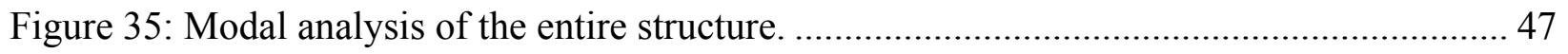

Figure 36: Acoustic model of the golf ball impact showing the acoustic point in space specified by node 90000 . 48

Figure 37: Acoustic model of the entire structure showing the microphone location (acoustic node 9000) 
Figure 38: Sound pressure from BEM analysis................................................................. 50

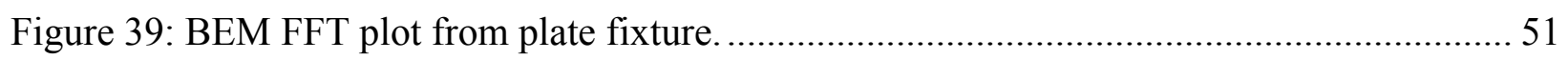

Figure 40: Microphone data from a "dryfire" of the aircannon.....................................................52

Figure 41: The pressure readings from the G.R.A.S. microphone of a 78 MPH impact.............. 53

Figure 42: FFT of the pressure readings from the microphone. ………………........................ 54

Figure 43: Zoomed in view of the FFT of the pressure readings.............................................. 55

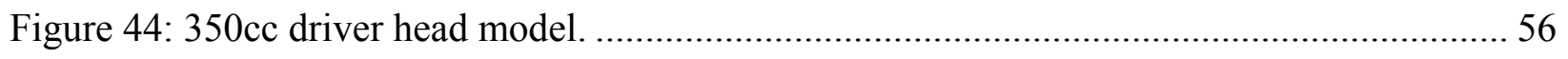

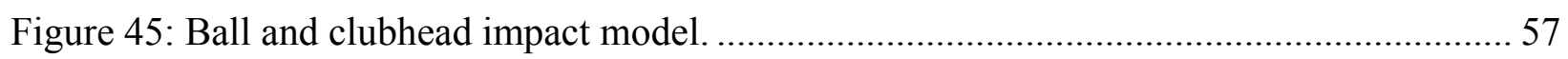

Figure 46: First mode oscillates around the shaft axis.......................................................... 57

Figure 47: The second mode oscillates towards "up" getting closer to the golfer's ear and

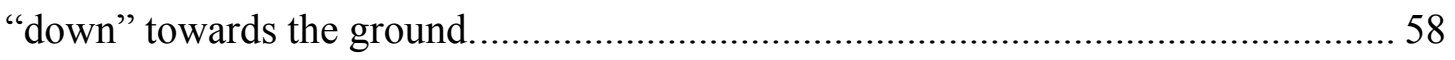

Figure 48: A frequency of $2508 \mathrm{~Hz}$ excites the face of the driver head. ....................................... 58

Figure 49: This mode excites the crown of the driver. ............................................................. 59

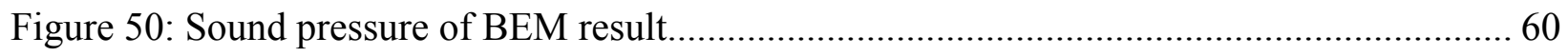

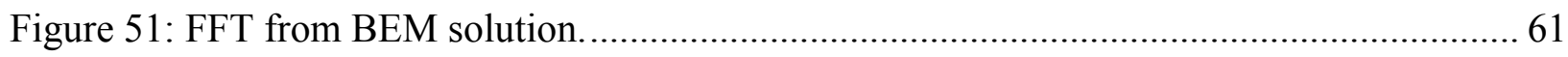

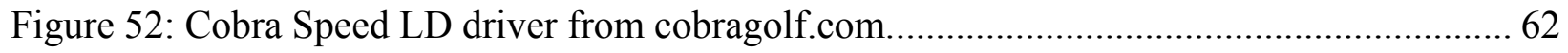

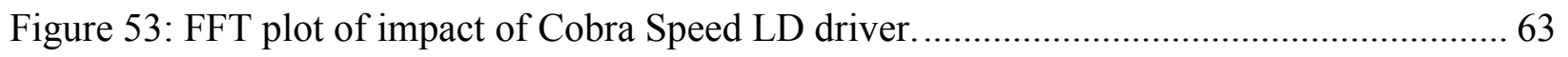

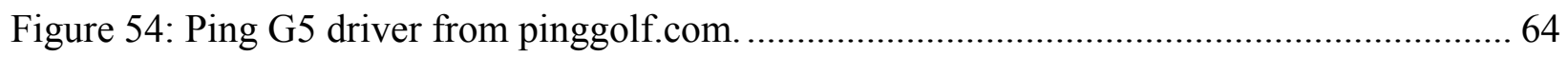

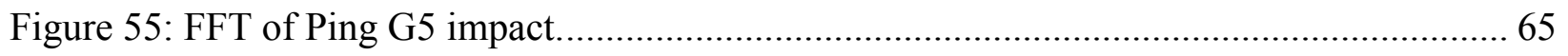

Figure 56: Cleveland Launcher DST driver from clevelandgolf.com. ......................................... 66

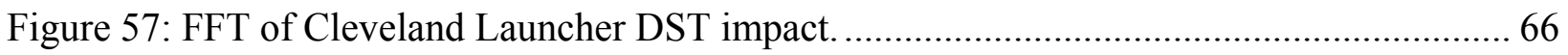




\section{Introduction}

\section{I.1 Motivation}

A golf driver head is made of three titanium components: the crown of the driver, a thin hemispherical shape on the top of the driver head; the sole, the thicker bottom piece, and the clubface, which is the most important part because it is the point where the ball impacts the driver head. The face is generally forged and electron beam welded to a cast titanium body. Thicknesses in each of the three components vary from point to point in the driver head, allowing a designer to dial in the optimal launch characteristics. All three components of the driver participate in producing a sound when impacted by a golf ball because of their distinct vibrational modes.

Most often a company prioritizes looks (marketing) and launch conditions as the primary design considerations. Throughout the design cycle, prototypes are made and tested to help better develop the product. As clubheads have gotten larger, approaching the $460 \mathrm{cc}$ limit, thin walls have made the sounds produced from some designs unpleasant to the ear. When a company designs a clubhead that performs well structurally, but has less than desirable sound quality, further design iterations are needed. In fact, one golf company recently spent three months of a twelve month design cycle working solely on a structurally sound clubhead [Mase]

When designing a club based upon acoustics there are two options: prototypes and simulations. A large company can front the cost of making prototype production units and measure the sound of impact, but it is not the most cost or time efficient. For the more computer savvy, the other option is to run a simulation. A computer simulation, when modeled correctly, can reproduce results which directly mirror real world outcomes. Without the aid of a golf club manufacturer, a 
simulation of the acoustical properties of the driver head is the best a single person or small team can achieve.

The sound heard from a professional golfer striking a golf ball is a combination of vibrational modes from the shaft, ball, crown, sole, and clubface after impact. These complex and sometimes convoluted shapes are what make it difficult for club designers to predict the sound of impact in early design stages. With no analytical way to determine the acoustics, one turns to numerical methods such as finite element analysis (FEA) and/or boundary element methods (BEM) to solve the problem. In Figure 1, the proposed problem is shown: an obscure shape is excited and produces sound waves that travel through the surrounding medium. The goal is to determine the best way to calculate the sound pressure waves so they can be played through a computer's speakers.

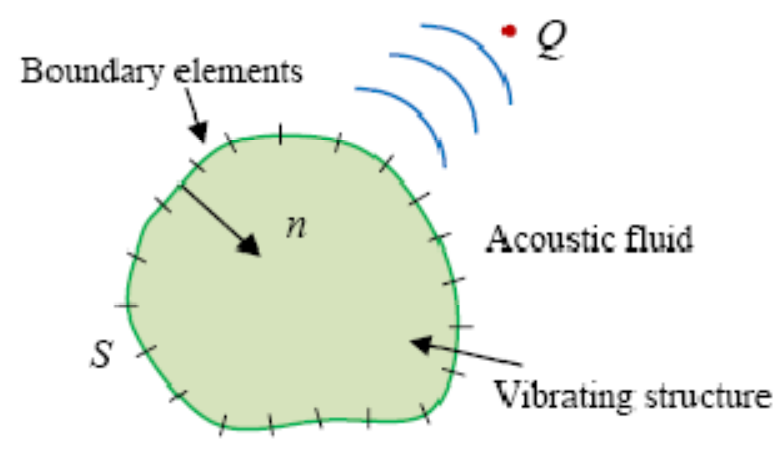

Figure 1: Definition of the problem, determining sound of an arbitrary object at a point in space. 


\section{Background}

\section{II.1 Acoustics History}

Linear acoustics is concerned with small amplitude phenomena. The most important equation, the acoustic wave equation, was derived by Helmholtz using state, continuity, and momentum equations as outlined in Springer's Handbook of Acoustics [Springer]. In the frequency domain, Helmholtz's equation is as follows:

$$
\Delta p+k^{2} p=0
$$

where $\mathrm{k}$ is the wave number defined by

$$
k=\frac{\omega}{C} .
$$

From these two equations, our pressure at any point, $\mathrm{p}$, is related to its pulsation frequency, $\omega$, and the speed of sound in the fluid, c. The combination of these two equations has led researchers to predict sound pressure waves from ideal spherical structures. The difficult part comes when our shape is not a sphere.

Wilton has presented a technique whereby using finite elements he was able to match the structure-fluid interface with an integral equation derived from Helmholtz's wave equation [Wilton]. It was used to determine the vibrational motion of the structure and also the associated acoustic field in the fluid. A similar approach was performed by Hunt in his mathematical model for acoustic scattering from an elastic structure immersed in a fluid [Hunt]. Both formulations used Helmholtz's equation and a Green's function to create a surface integral equation.

A simpler adaption would be to use boundary elements to solve for the acoustic pressure at a specific point. As outlined by Springer Handbook of Acoustics [Springer] in order to apply the BEM to the Helmholtz equation, one of Green's theorems must be used to reduce Helmholtz's 
equation to an integral (weak form). Alia and Souli demonstrate that when Helmholtz's equation is combined with Green's second identity, the result is an integral equation which can be used to solve for the pressure at any field point [Alia]

$$
\begin{gathered}
G r, r_{y}=\frac{e^{i k r-r_{y}}}{4 \pi r-r_{y}}, \\
C r p r=-{ }_{S_{y}} \mid i \rho \omega v_{n} r_{y} G\left(r, r_{y}\right)-p\left(r_{y}\right) \frac{\partial G r, r_{y}}{\partial n_{y}} d S_{y} .
\end{gathered}
$$

On the surface of the acoustic boundary, the pressure is related to the structural velocity by:

$$
\nabla p=-i \omega \rho v
$$

In the BEM, this means only the radiating surface needs meshing whereas with the finite element method the fluid medium also needs discretization. LS-DYNA ${ }^{\circledR}$ has been able to utilize the finite element analysis of a structure and turn it into a boundary element mesh of the surrounding fluid. This can be a very computationally intense process. A general rule of thumb from Springer states the solution time for FEA is proportional to the number of nodes squared while the BEM solution time is proportional to the number of nodes cubed [Springer].

Another option when solving an acoustic problem is to use Rayleigh's method to determine the sound pressure levels. While it is technically not widely accepted as an accurate method for sound pressure levels by Smith [Smith] there is a significant savings in computational time. LSTC indicates the BEM is very effective for coarse meshes under a total of 2000 boundary elements, but warning that the BEM remains computationally and resource heavy since it involves iterating for every required frequency [Huang]. On the other hand, Rayleigh method can directly solve for a solution without iteration. 
Rayleigh method is based on the assumption that each element of the vibrating surface is mounted on an infinite rigid baffle and vibrates independently from the other elements on the surface. Therefore, the total pressure field is obtained by summing the pressure generated by each element. The resulting half-space Green's function in Equation 6 is the first step in determining the Rayleigh integral.

$$
G_{H}\left(r, r^{\prime}\right)=\frac{e^{-i k r}}{4 \pi r}+\frac{e^{-i k r^{\prime}}}{4 \pi r^{\prime}} .
$$

Now, if the vibrating surface lies in the half-space plane, the Green's function and partial derivative reduce to

$$
G_{H}(r)=2 G(r)
$$

and

$$
\frac{\partial G_{H}(r)}{\partial n}=0
$$

The final equation reduces to

$$
p r={ }_{S_{y}} 2 i \rho \omega v_{n} r_{y} G_{H}\left(r, r_{y}\right) d S_{y} .
$$

The result is the sound pressure at any point can be easily obtained by the normal velocities on the surface. Also because there are no matrices to invert and solve for at each frequency, the computational time is very short when compared to the BEM.

\section{II.2 Metal Woods}

The sport of golf has changed from the days of the "thwack" from a persimmon driver to the "ting" of modern titanium drivers. The generic shape of a driver is displayed in Figure 2. The 
progression from persimmon to metal started in the late 1970's when TaylorMade, one of the first companies to do so, marketed a driver made of metal. These early revisions of the metal wood did not catch on very quickly because the size and performance gains were minimal, if not none, when compared to persimmon drivers of the time period. It wasn't until the 1990's when the coefficient of restitution (COR) became an integral part of driver head designs that metal woods took off.

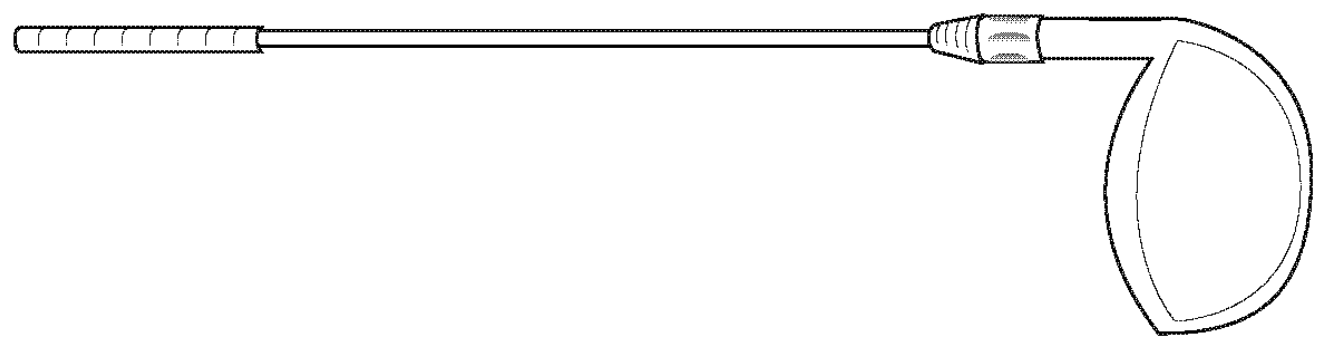

Figure 2: Generic golf club (not to scale).

\section{II.2.1 History}

TaylorMade produced the first metal golf driver heads in the late 70's. The nicknamed "Pittsburgh Persimmon" club was constructed of steel and secured its first PGA tour victory in 1981 [TaylorMade]. These initial designs did not offer significant performance gains over the persimmon wood-driver norm of the period, yet other major golf manufacturers soon followed suit with their own metal diver head designs. While stainless steels, aluminum alloys, and carbon 
fiber have been used in the construction of driver heads, the most popular and accepted material is titanium due to its low weight and high strength material properties. In Table 1 most of the popular materials are displayed along with their Young's Modulus and density. TaylorMade introduced the Ti Bubble, its first titanium driver in 1996. The use of titanium allowed the size of the head to increase and the face of the driver to become thinner all while maintaining the same weight as persimmon drivers. Thusly, as the performance of metal woods increased, so did their popularity.

Table 1: Common metals used in driver head construction and their properties from Matweb.com.

\begin{tabular}{|ccc|}
\hline Material & Young's Modulus, $\mathbf{E}(\mathrm{msi})$ & Density, $\rho\left(\mathrm{lb} / \mathrm{in}^{3}\right)$ \\
\hline Ti-6Al-4V & 16.510 & 0.160 \\
\hline Ti-15V-3Cr-3Al-3Sn & 11.900 & 0.172 \\
\hline $\mathbf{3 0 4 S S}$ & 28.000 & 0.289 \\
\hline $\mathbf{1 7 - 4 P H}$ & 28.600 & 0.280 \\
\hline
\end{tabular}

\section{II.2.2 Design}

Discussion about performance gains often centers on coefficient of restitution (COR) and moment of inertia (MOI). With a drastic increase in distance from the trampoline effect of thin walled driver faces, the United States Golf Association (USGA) along with the Royal \& Ancient Golf Club of St. Andrews (R\&A) declared a COR of 0.830 as the maximum in addition to a maximum volume of 460 cubic centimeters. Drivers were designed with thin faces of $3 \mathrm{~mm}$ or 
less and the size of the club face pushed to the maximum allowable dimension their $460 \mathrm{cc}$ frames could allow. While there may be a lot of thought put into the design of a driver beyond distance gains, a customer can only judge the club in three categories: aesthetics, feel, and sound.

\section{II.2.3 Sound Production}

A golf driver head is a complex structure which vibrates in numerous ways. As previously mentioned, the sources of sound producing vibration come from; the driver, the golf ball, and the golf shaft. A more complex analysis can bring all three components into the simulation, but we are mostly concerned with the driver head itself. The face of the driver is the first component to come into contact with the golf ball. The resulting stress wave propagates out from the point of impact across the club. In the more common $460 \mathrm{cc}$ drivers of today, the crown of the driver is both the largest and thinnest piece of the driver and is a key component in the perceived sound of impact due to the fact the normal of the surface is pointed towards the ears of the golfer. The thickest and stiffest part of the driver, the sole, does most of the mechanical bracing and contains the majority of the mass of the driver head. The way the sole braces the internal structure of the clubhead greatly affects the mode shapes produced and consequently the perceived sound.

\section{II.3 Golf Balls}

Golf balls are a major component to the sound of impact since the ball provides the excitation to the clubhead. The construction of the club and point of impact of the ball on the face of the club are the major factors in sound production. A "mishit" will produce a sound which allows many experienced golfers to identify the shot as poorly struck. Also, using a firmer, two-piece 
ball designed for distance will change the sound produced at impact. A stiffer ball will excite a broader spectrum of sound, acting more like a Dirac Delta impulse function. The stiffness of golf balls can vary 100 percent between premium 3-piece ball and a value 2-piece ball [Mase]. Deciding which type to play with usually comes down to the perceived skill of the golfer.

\section{II.3.1 History}

Just as golf club technology has progressed, the same can be said for golf balls. The history of the golf ball starts with the Featherie. The Featherie is sack of leather packed with feathers and sewn to form the general shape of a ball. The Guttie, given its name from being made of a solid piece of gutta percha, was the next revolution in golf ball technology. Stumbling upon the aerodynamic effects of a cut-up golf ball changed how Gutties were constructed and dimples were born [Golf Europe]. The golf ball has been refined from the gutta percha by makeshift aerodynamicists throughout the centuries. The general design of a dimpled cover and rubber core has been a patented design that is the standard for golf balls today.

\section{II.3.2 Design}

Typical construction of a golf ball today consists of either a two-piece ball or a multilayer performance ball. The cheaper two piece balls have a single material for the core and an ionomer resin for the cover. The more expensive multiplayer performance balls have a core and multiple inner mantles wrapped in a soft urethane cover to yield the best overall distance and spin characteristics [Mase].

\section{II.3.3 Sound Production}

The ball undergoes its own vibration modes that contain both acoustic and silent vibrational modes [Axe]. The silent vibrational modes are caused by a tangential force on the cover of the golf ball. Acoustic modes are caused by a combination of radial and tangential forces causing the 
outer cover to oscillate outside of the 1.68 inch diameter sphere of the golf ball. This oscillation produces a pressure wave and sound is created.

The vibrational modes of the ball are excited at much lower frequencies than the clubhead due to the materials used in the construction of the golf ball. When dropping a piece of rubber and a piece of titanium on the floor, which one makes more of a sound? For purposes of this thesis the sound produced by the golf ball has not been considered and no attempt was made to extract the golf ball sound from the finite element model.

\section{II.4 Methods to Determine Sound Performance}

Driver clubheads are diverse in nature, from their geometry to their material. Every year companies produce different designs so they can have a "new" model in the marketplace. In a sense, this makes the driver market a fashion focused market. The diverse clubhead designs result in an equally diverse set of sounds the clubs produce. While we know the different parts of a golf club can make a sound, it is sometimes problematic to characterize these sounds. In Figure 3, a laser holography photo indicates the modes that make up the distinct sound of impact in a Callaway Big Bertha driver head. 


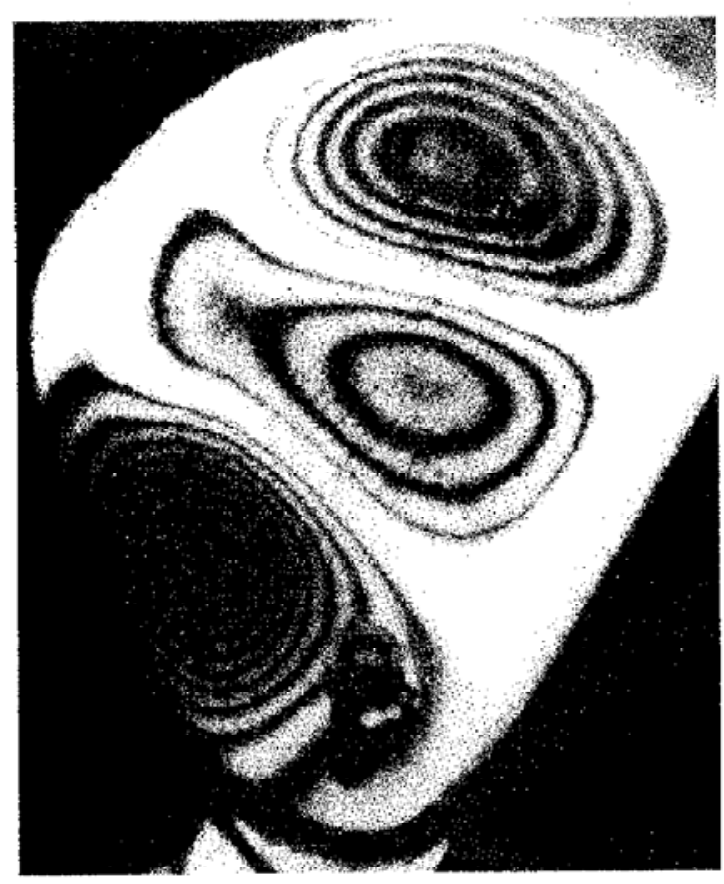

Figure 3: Laser holography picture of the sound produced from a Callaway Big Bertha driver.

\section{II.4.1 Field Testing}

The easiest and least scientific method is to put the product out in the field, have people play it, then provide feedback. This means that some of the most influential people in the design of a club are touring professionals. They are the ones who rely on the equipment day to day and can give much more feedback than the general public. These player testimonials are very important in the development of the product. When using player testimonials, many of the significant variables like ball type and shaft manufacturer are not factored in and therefore, the more regimented testing method is the field study.

In the field study, many of the variables used in player testing are now standardized. The type of ball will be specified and the shaft options will be those supplied by the manufacturer. But again, the player testimonial is used to evaluate driver performance.

\section{II.4.2 Direct Testing}


A more scientific method to determine the sound output involves using either a swing robot or highly skilled golfer to hit golf balls within close proximity to a microphone. The sound recorded can then be analyzed by performing a Fast Fourier Transform (FFT) of the input signal. The result will be a plot of frequency and magnitude of the sound pressure waves. From here "good" and "bad" frequencies can be distinctly determined. The bad sounds are objective and come down to the designer or golf company. An optimization routine used during development can steer a design away from a "bad" sound by either lowering the frequency magnitude in the FFT plot and/or to shift the frequency to a more desirable range.

\section{II.4.3 Computer Modeling}

In general, the purpose of computer modeling is to create something digitally that recreates or mimics real world behavior. For this project, there are many stages that need to be completed before a final sound file can be produced. The steps used to run the simulation can be found in Figure [4]. 


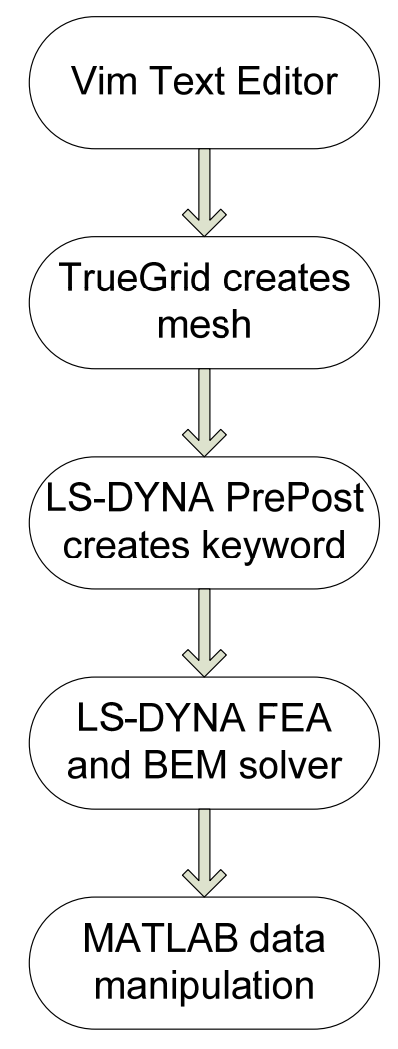

Figure 4: Flow of programs used to create simulated impact sound.

In any finite element solution, there are basic, common steps which need to be done. First a mathematical model needs to be produced to characterize the physical problem. This includes assumptions in: geometry, loading, boundary conditions, material laws, etc. The model is made up of a mesh of finite elements, where each element has nodes that represent the connection inside the mesh. The finite element method is an approximation, and therefore accuracy of the solution depends on the discretization of the mesh. It is best to run a coarse mesh and systematically refine it until the accuracy of the solution is within the desired range. Once the mesh is sufficiently refined, a more complex loading or boundary condition may be applied and the results interpreted. If the analysis needs to be refined to improve the mathematical model it can be done at this time [Sharpe]. The end result should be an accurate model that will help in the design and optimization of the physical problem. 
TrueGrid ${ }^{\circledR}$ was used for its ability to create superior meshes and output the mesh in LSDYNA ${ }^{\circledR}$ keyword format. TrueGrid ${ }^{\circledR}$ is a very powerful software program which can create meshes on odd shaped 3D parts from other drawing programs. In this case, simple commands were used to create the structures used in the analysis. Snippets of the code used to generate the structures can be found in Appendix B along with the final input files. The general idea is one first must create an index space which defines the total number of elements seen in any of the x-, y-, or z-directions. Next, a surface is called out, either planar or a curve (even a 3D scan of a bone has been used), that the index is mapped too. If this is the only part created, the mesh is done. Problems occur when multiple parts are merged together. It is important to ensure the nodes coincide at the same point in 3D space or the two parts will act independently of each other, giving false readings. Accounted for in the input files are scaling factors which allow one to increase the mesh density by increasing a coefficient. It is possible to incorporate boundary conditions and load cases in the TrueGrid ${ }^{\circledR}$ input file, but is easiest to take the created keyword file from TrueGrid $\AA$ and open it in LS-PREPOST ${ }^{\circledR}$ for manipulation.

LS-DYNA ${ }^{\circledR}$ is superior finite element software that has been used extensively for impact simulations. Attached with LS-DYNA ${ }^{\circledR}$ is its pre and post processor, LS-PREPOST ${ }^{\circledR}$, where through the graphical user interface (GUI) one can modify the keyword file as needed. Important nodes can be chosen and put into node sets that will be used to define boundary conditions. Also, key points where a structure interacts with a fluid to produce a sound can be put into sets using the GUI. These sets are how the BEM solver knows which nodes contain the important surfaces used in the simulation. In the *BOUNDARY card, the BEM solver is found under ELEMENT_METHOD_ACOUSTIC. 
Recently, with the newest release of the software, a coupled finite element and boundary element solver is implemented. This solver can take the results of a finite element mechanical simulation and use information about nodal velocities to determine how sound waves will propagate to predetermined acoustic points. The flow of the solver is shown in Figure 5.

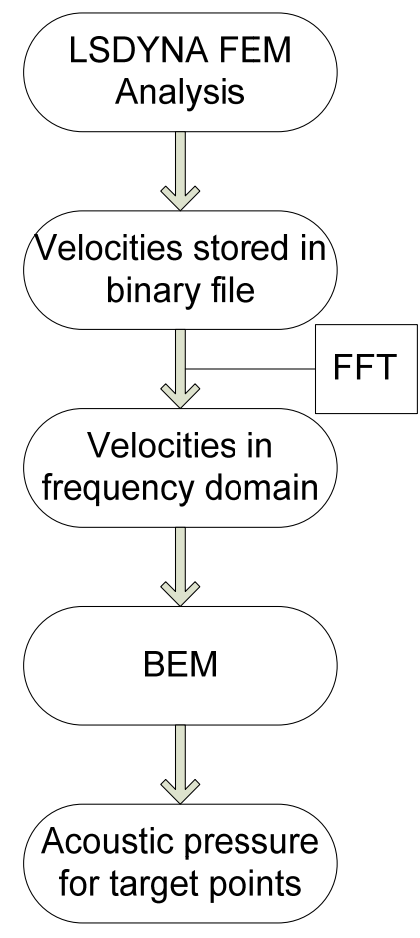

Figure 5: Flow of operations performed by LS-DYNA ${ }^{\circledR}$ in the coupled FEA/BEM solver.

The boundary element card from a LSTC publication is shown in Figure 6. 


\section{Card Format}

\begin{tabular}{|c|c|c|c|c|c|c|c|c|}
\hline Card 1 & 1 & 2 & 3 & 4 & 5 & 6 & 7 & 8 \\
\hline Variable & Dens & Sp-sound & Min_Freq & Max_Freq & NFreq & Dt_Output & Start_Time & Ref_Pres \\
\hline Type & $\mathrm{F}$ & $\mathrm{F}$ & $\mathrm{F}$ & $\mathrm{F}$ & I & I & $\mathrm{F}$ & $\mathrm{F}$ \\
\hline Default & none & none & none & none & 0 & 0 & 0 & 0 \\
\hline Remark & & & & & & & 1 & 2 \\
\hline
\end{tabular}

\begin{tabular}{|c|c|c|c|c|c|c|c|}
\hline Card 2 & 1 & 2 & 3 & 4 & 5 & 6 & 7 \\
\hline Variable & Ext_Pres & Type_Ext & Int_Pres & Type_Int & FFT_Wind & & \\
\hline Type & I & I & I & I & I & & \\
\hline Default & 0 & 0 & 0 & 0 & 0 & & \\
\hline Remark & & & & & 3 & & \\
\hline
\end{tabular}

\begin{tabular}{|c|c|c|c|c|c|c|c|c|}
\hline Card 3 & 1 & 2 & 3 & 4 & 5 & 6 & 7 & 8 \\
\hline Variable & IBEM_Met & Max_Iter & Res & NDD & & & & \\
\hline Type & I & I & $\mathrm{F}$ & I & & & & \\
\hline Default & 0 & 100 & $10^{-6}$ & 1 & & & & \\
\hline Remark & 4 & & & 5 & & & & \\
\hline
\end{tabular}

\begin{tabular}{|c|c|c|c|c|c|c|c|c|}
\hline Card 4 & 1 & 2 & 3 & 4 & 5 & 6 & 7 & 8 \\
\hline Variable & SSID & SSTYPE & Norm & BEM_Type & Restart & & & \\
\hline Type & I & I & I & I & I & & & \\
\hline Default & 0 & 0 & 0 & 0 & 0 & & & \\
\hline Remark & & & & & 6 & & & \\
\hline
\end{tabular}

Figure 6: Boundary element card variable description from LSTC press release.

The first two variables are the most important in the analysis. The density of the medium the sound is traveling in needs to be in the same unit system as the speed of sound. The "F" and "I" seen under "Type" specifies whether the solver is looking for a floating point number or an integer. The integer is used as a flag in the code for different options, while the floating point number is used as a variable in the solution. A problem arose when under Dt-Output trying to put a value in LS-PREPOST ${ }^{\circ}$. The graphical user interface will only allow an integer in that box when it needs a floating point number and must be manually changed in the keyword later by using a text editor. The start time can be specified, and is helpful in catching only the sounds 
created after an impact. The reference pressure is used to create the pressure output graph in decibels and can be omitted if not needed.

Under card 2, the acoustic points are specified by what type they are and whether they are internal or external. The FFT window option contains all the standard windowing functions such as Rectangular, Hanning, Hamming, etc. There are some hidden features not in the GUI or the press release. For example, in the $6^{\text {th }}, 7^{\text {th }}$, and $8^{\text {th }}$ columns of card 2 there are three missing features. These features include an option to display the time domain results, more pressure output files, and an automatic unit change. Sample Keyword files containing the BEM code and how it's used are in Appendix C.

In card 3, the choice of three solvers falls under IBEM_Met. The menu includes: the full BEM solver, the Kirchoff method (which is coupled with *MAT_ACOUSTIC), and the Rayleigh method outlined earlier. The max iterations and residual are used only for the BEM solver since it is the only one that cannot be directly solved. The Number of Domain Decomposition (NDD) can be utilized to save memory for larger problems. All these options were optimized for our specific problem and the simplified LS-DYNA® keyword file used for this simulation can be found in Appendix C. 


\section{Plate Validation}

The goal of this thesis was to simulate the sound of a clubhead when impacting a golf ball. However, when using a new type of software, it is best to validate the findings from the simulation using analytical or experimental results. In the case of golf club acoustics, a circular plate was much easier to model and validate. In our case, Titleist graciously donated a titanium plate they previously used for COR testing to use as our validation test. An air cannon was built to fire golf balls at the titanium plate braced by a support structure made of T-slot extrusions. Acoustic and strain data were collected from this setup and used to validate the finite element mesh and model results.

\section{III.1 Experimental Setup}

Since we are trying to validate the acoustic output of a titanium plate, two structures needed to be built. First, we needed something to fire a golf ball at specific velocities with enough precision to hit a target not much larger than the golf ball itself. Secondly, a chamber needed to be created which would brace the plate during impact and also house a microphone to gather acoustical data. Cal Poly did not have any resources like that available, therefore both were designed from scratch and can be seen in Figure 7. 


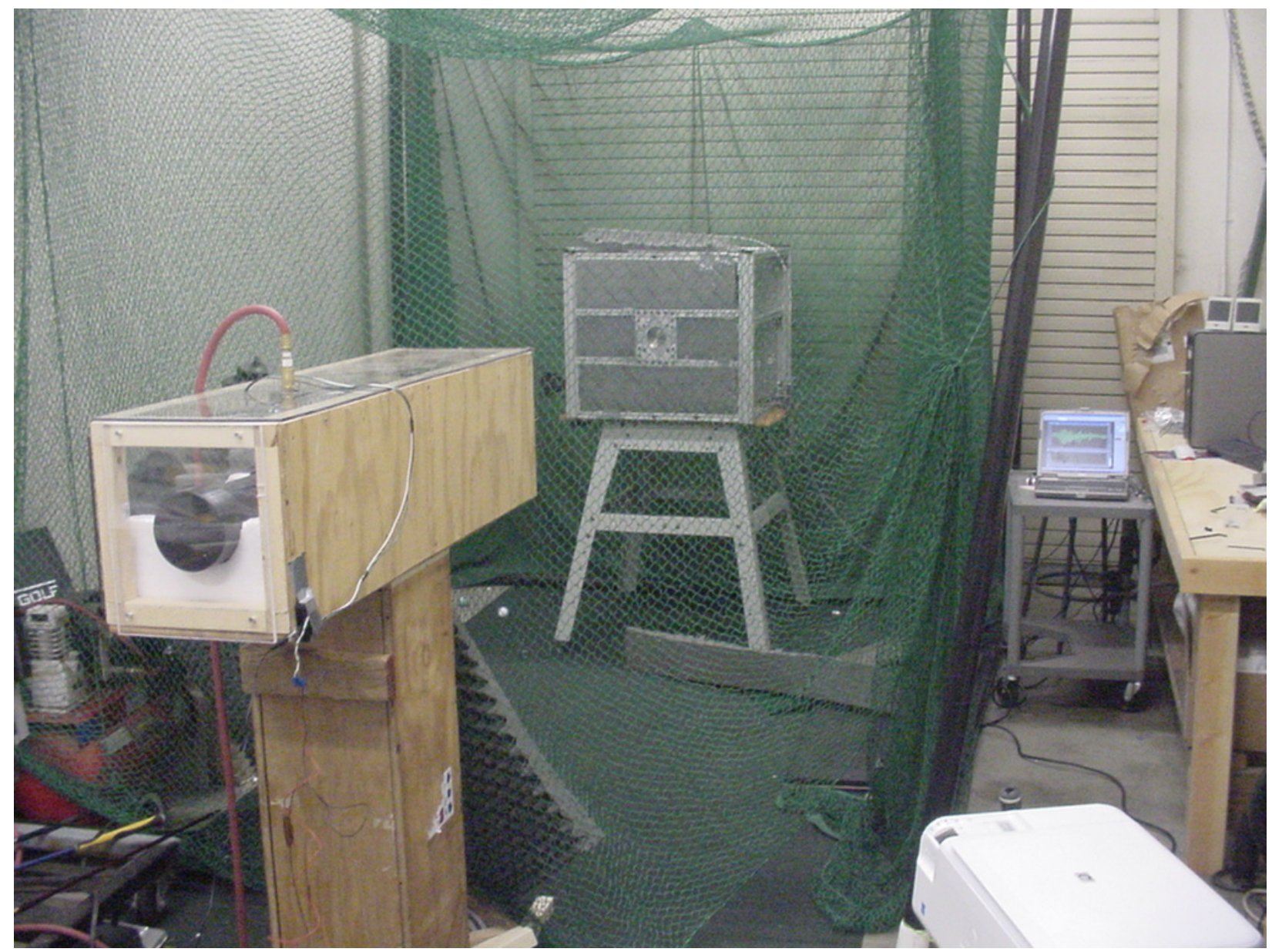

Figure 7: Experimental setup to determine the sound output of the titanium plate. Shown is the air cannon, plate support structure and data acquisition system.

\section{III.1.1 Air Cannon Build}

A vital component of the experiment was the air cannon. We needed a way to fire a golf ball at high velocity with enough accuracy to consistently hit an area the size of a dime located within a three inch diameter opening. The air cannon design consisted of three main components: a storage chamber, solenoid, and rifle tube. When we could, all parts were made of ABS or PVC plastic to reduce cost. Air was the working fluid for this experiment and was compressed in the storage chamber. 


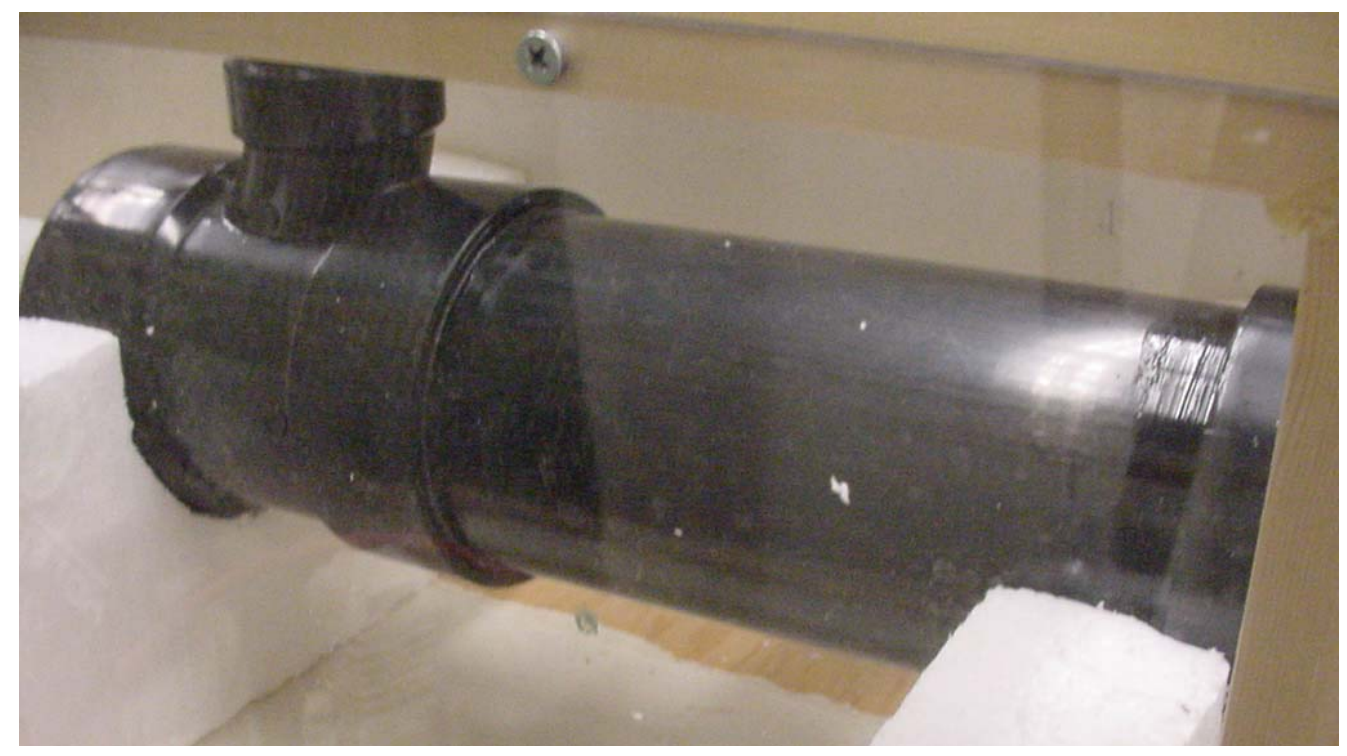

Figure 8: Storage chamber of the air cannon.

The storage chamber was modeled as a cylinder with a four inch inner diameter and six inch length and can be seen in Figure 8. This would be enough air volume for a single fire. A simple work-energy analysis performed on the storage chamber and rifle tube provided an estimate of exit velocity. Summing forces on the ball gives two acting forces, the pressure from the storage chamber and atmospheric seen in Eq 10.

$$
\sum F=A_{\text {Golf Ball Cross-Section }} P_{\text {Storage Chamber }}-P_{\text {Atmospheric }}
$$

An assumption can be made about the pressure in the storage chamber while the ball moves through the rifle. Previous lab research [Sharpe], using a polytropic constant in Eq 11 can give an estimate of the pressure reduction in the rifle tube

$$
\begin{aligned}
& P V^{1.4}=\text { constant } \\
& \int P d V=\frac{1}{2} m v^{2}
\end{aligned}
$$


In Eq 12, the bounds on the integral are the volumes at the initial and final states. The velocity term is simplified because the initial velocity of the golf ball is zero. The constant in Eq 11 is solved for at the initial state as a function of pressure and then put into Eq 12. The resulting Eq 13 can be solved to determine an estimate of the exit velocity of the golf ball.

$$
\int_{V_{1}}^{V_{2}} \frac{\text { constant }}{V^{1.4}} d V=\frac{1}{2} m_{\text {Ball }} v_{\text {exit }}^{2}
$$

From this simple work-energy method, the exit velocity calculated was $113 \mathrm{mph}$ at $30 \mathrm{psi}$ in the storage chamber. Of course the actual velocity will be lower due to the ball not completely sealing the rifle tube and the redirection of flow through the orifice of the solenoid, but by simply knowing the pressure inside the storage chamber a theoretical exit velocity can be determined.

An air pressure sensor was attached to the storage chamber and can be seen in Figure 9. The pressure sensor was critical when determining a pressure velocity correlation. When the storage chamber was up to the correct pressure, the solenoid valve was opened. 


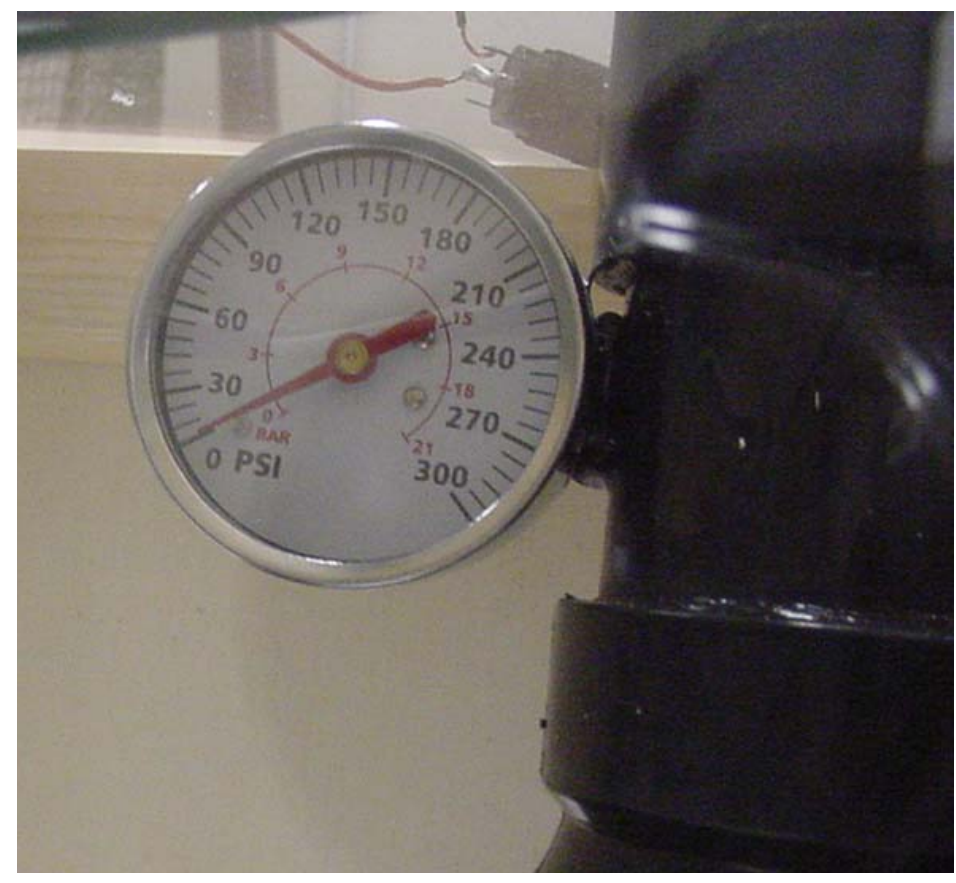

Figure 9: Air pressure sensor connected to the storage chamber.

As the gateway between the storage chamber and rifle tube, the solenoid was the most important piece of the entire air cannon. The solenoid has a one inch diaphragm and is a pilot operated valve activated by a twenty-four volt direct current supple voltage. The internal workings of the solenoid valve can be found in Figure 10a compared to the installed solenoid in Figure 10b. It was purchased from McMaster Carr that had a $12.9 \mathrm{Cv}$ factor rating. The $\mathrm{Cv}$ factor is the coefficient of volume and is the amount of liquid, in gallons, per minute that passes through a fully open valve at a one psi differential pressure [McMaster]. After flowing through the diaphragm, the air pressure hits the back of the stationary golf ball in the rifle tube. 

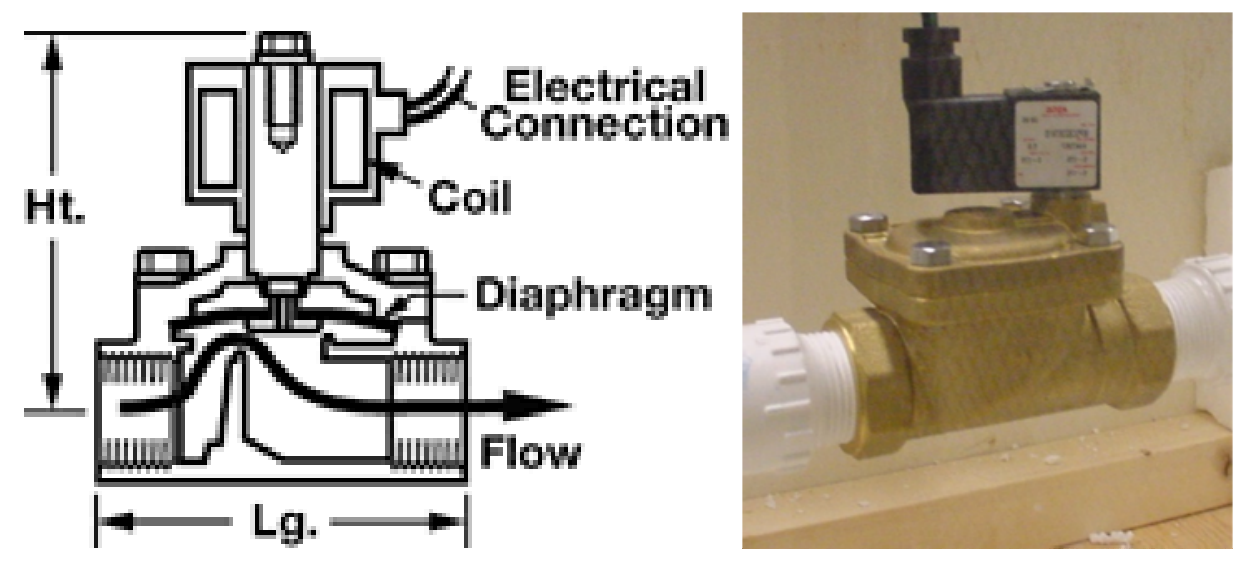

Figure 10: Solenoid (a) cross-section from McMaster and (b) in use.

For the golf ball to reach maximum velocity the clearance between the golf ball and walls of the rifle tube had to be very tight. A schedule 1201 1/4" pipe was used that had a inner diameter of 1.70 inches which closely matches the outer diameter mandated for a golf ball by the USGA

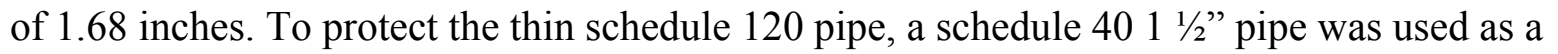
sleeve. When all the components were attached and wired correctly with sufficient safety precautions, we had a functioning air cannon which can be seen in Figure 11. 


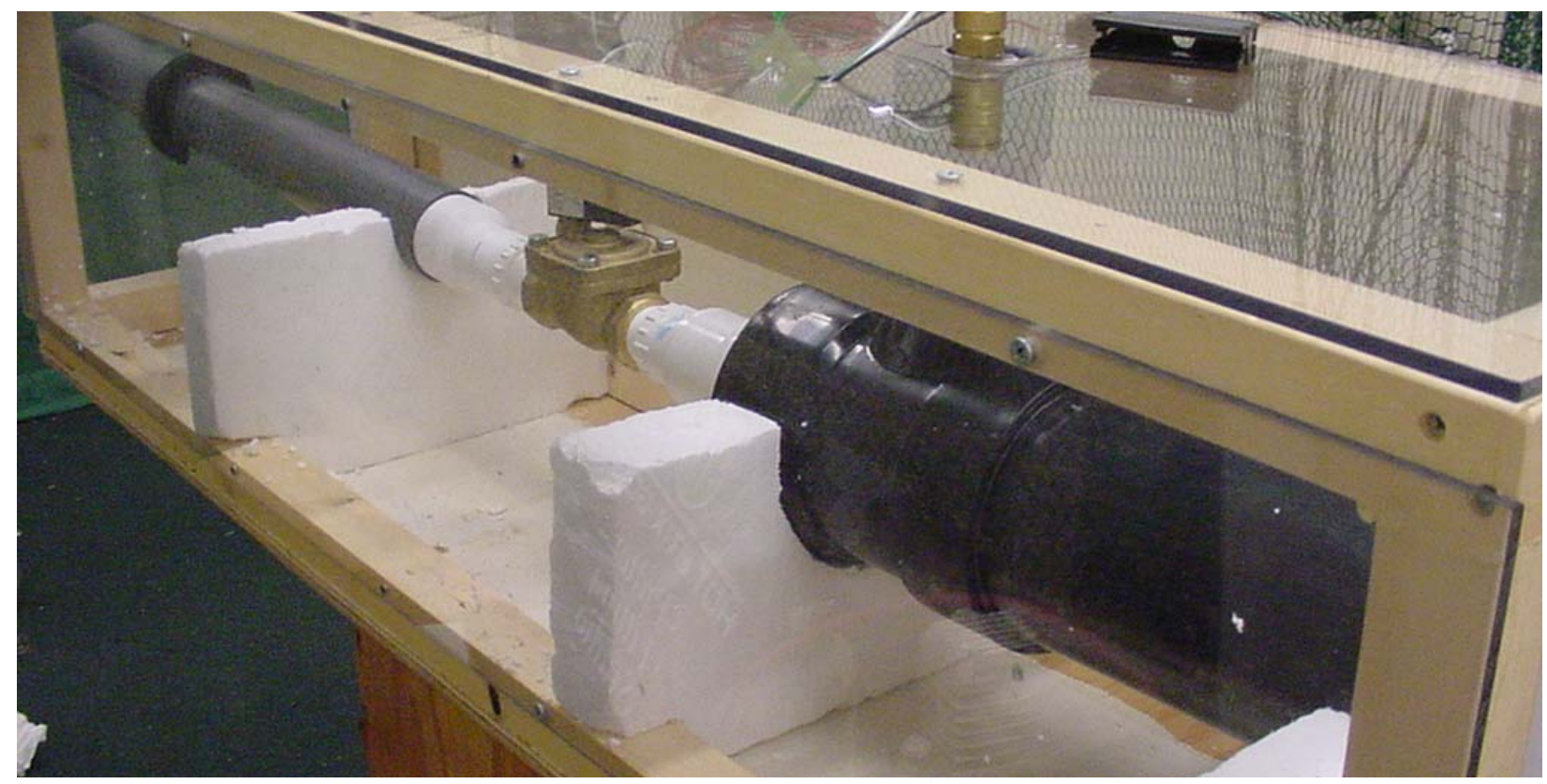

Figure 11: Air cannon placed on test stand behind 1/2" polycarbonate.

\section{III.1.2 Air Cannon Calibration}

The air cannon was built, but still needed to be calibrated. We needed to know what speeds at the end of the tube coincided with pressure readings in the storage chamber. Using a Cal Poly designed golf ball launch monitor, we attempted to measure the golf ball exit velocity. The launch monitor functions by measuring the sound of impact using a microphone and triggering a timed strobe of light while the shutter of a digital camera is open. In this case, there was no sound from impact, only from the solenoid dumping air in the chamber. We tried setting up a delay in the trigger using the accompanying software, but the timing was so inconsistent and difficult to dial in that other means were necessary in order to determine a pressure velocity curve. The next step was to use a high speed camera. 


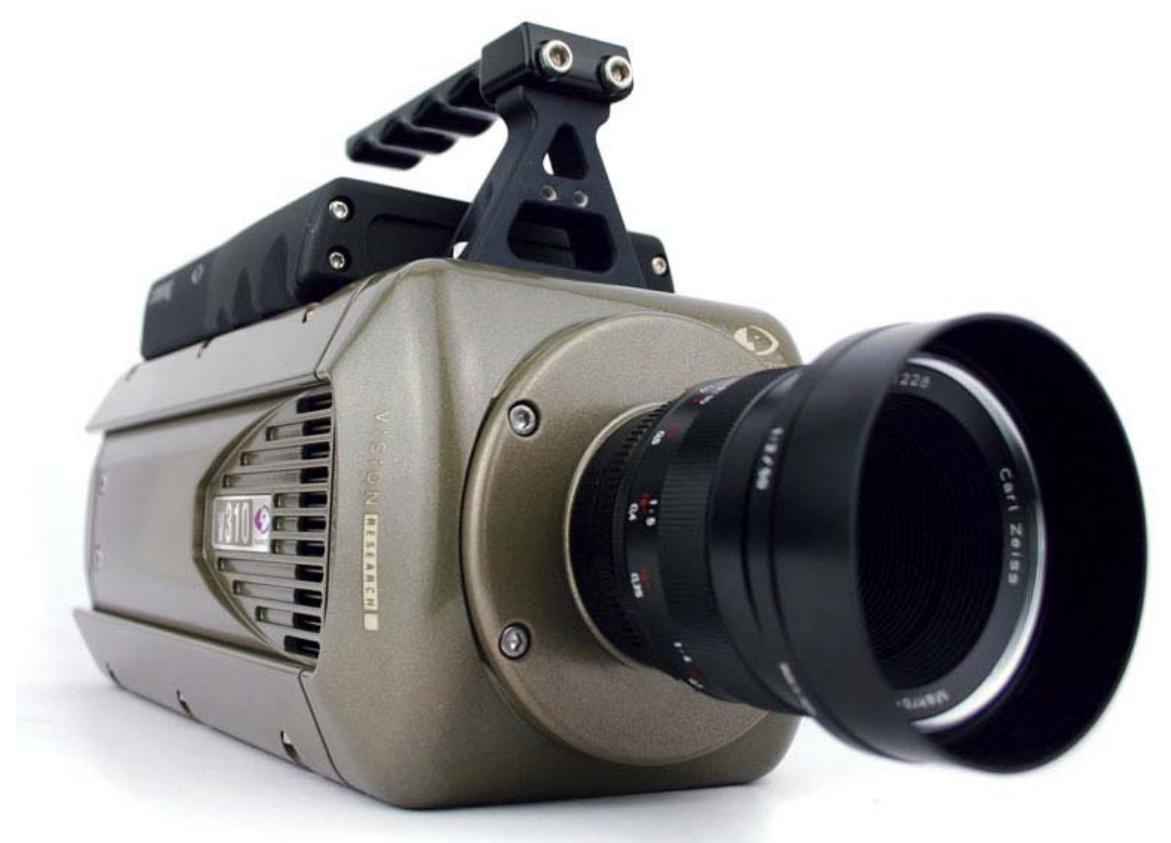

Figure 12: Phantom v310 high speed camera from visionresearch.com

Thankfully, the Mechanical Engineering department at Cal Poly has a high speed camera available for students to use. A Phantom v310 high speed camera, seen in Figure 12, was used to capture the ball exiting the tube against a background grid. The high speed images were recorded at 7500 frames per second against a grid with half inch striations. After recording the shot, the video could be loaded with the accompanying software and using the grid, the velocity of the ball could be determined. Using the high speed camera along, a calibration curve was determined for the air cannon and can be seen in Figure 13. The resulting Eq 14 from a best fit curve can give a good approximation of the exit velocity of the golf ball.

$$
\text { Exit Velocity } m p h=1.1175 \times \text { Storage Pressure } p s i+27.682
$$




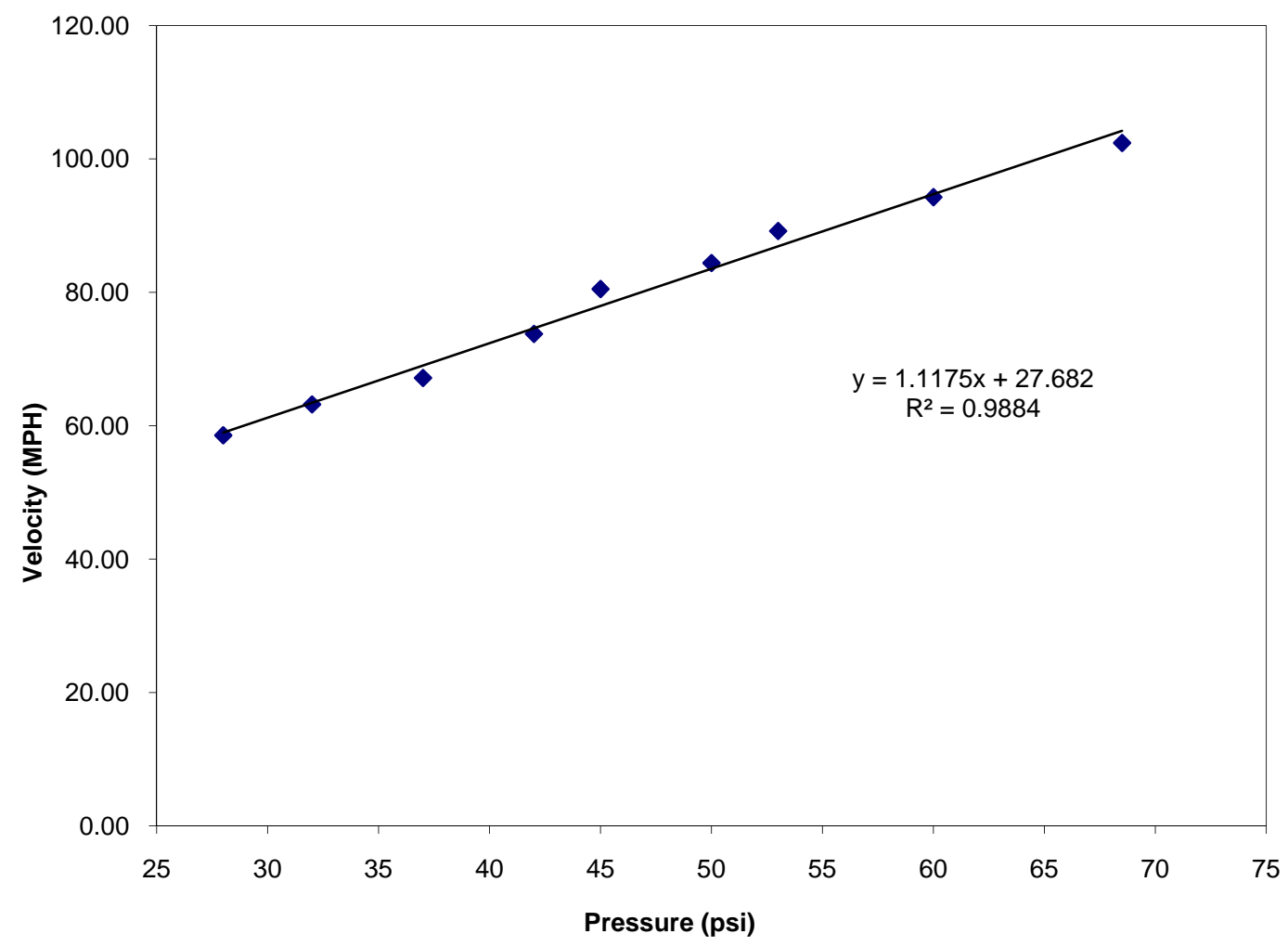

Figure 13: Calibration curve for the air cannon.

An issue with any object being fired down a long tube is spin. A ball with spin is more difficult to model because it will induce a stress in the plane of the plate. Using the high speed camera, it was possible to watch the rotation of the golf ball in the air. In Figure 14, the ball in mid flight is shown and analysis of the video showed little to no rotation of the golf ball. 


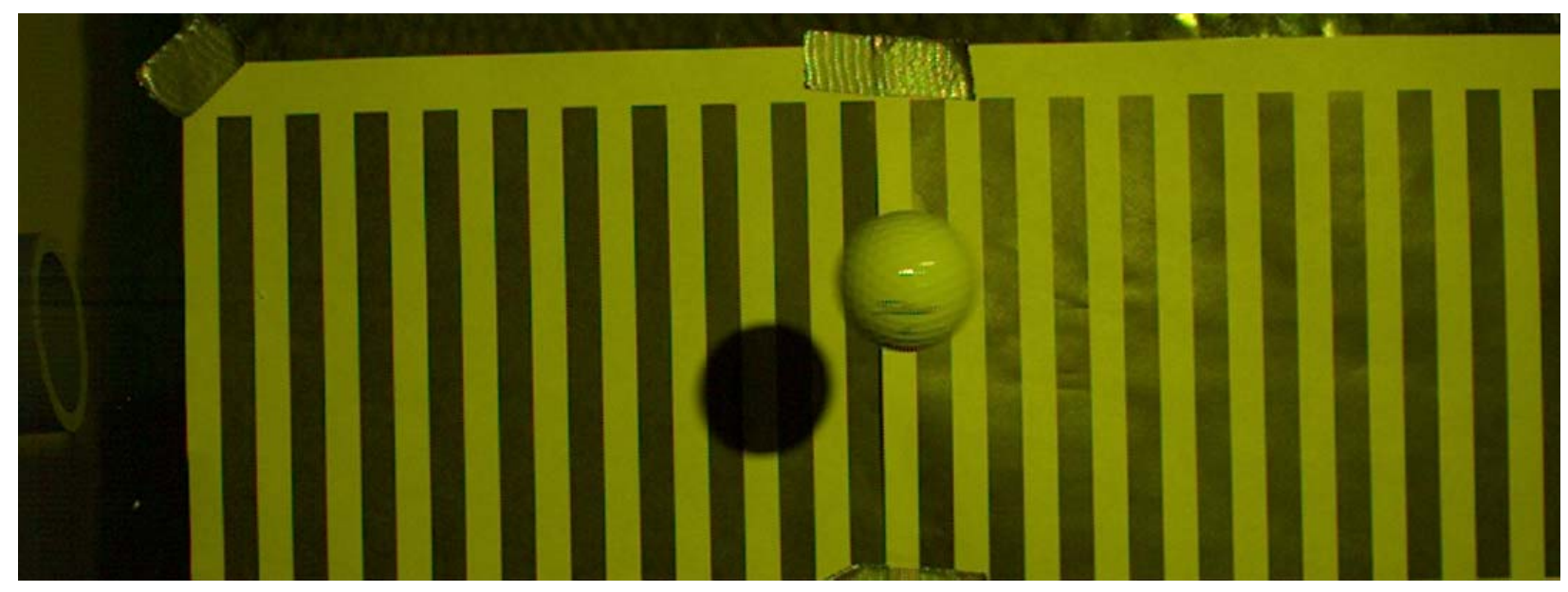

Figure 14: Titleist ProV1 golf ball exiting the air cannon with little to no spin.

\section{III.1.3 Plate Fixture}

The most critical part on the validation experiment was the fixture that holds the titanium plate. This structure was designed to be very stiff and able to withstand an impact from a stray golf ball. The result was a structure made entirely of T-slot extrusions. T-slots were chosen because they allow the structure to be adjusted to the specific height of the air cannon. If the golf ball missed the desired target by a half an inch, the supports could be unscrewed and the plate moved as necessary relatively easily. The picture of the plate structure can be found in Figure 15. 


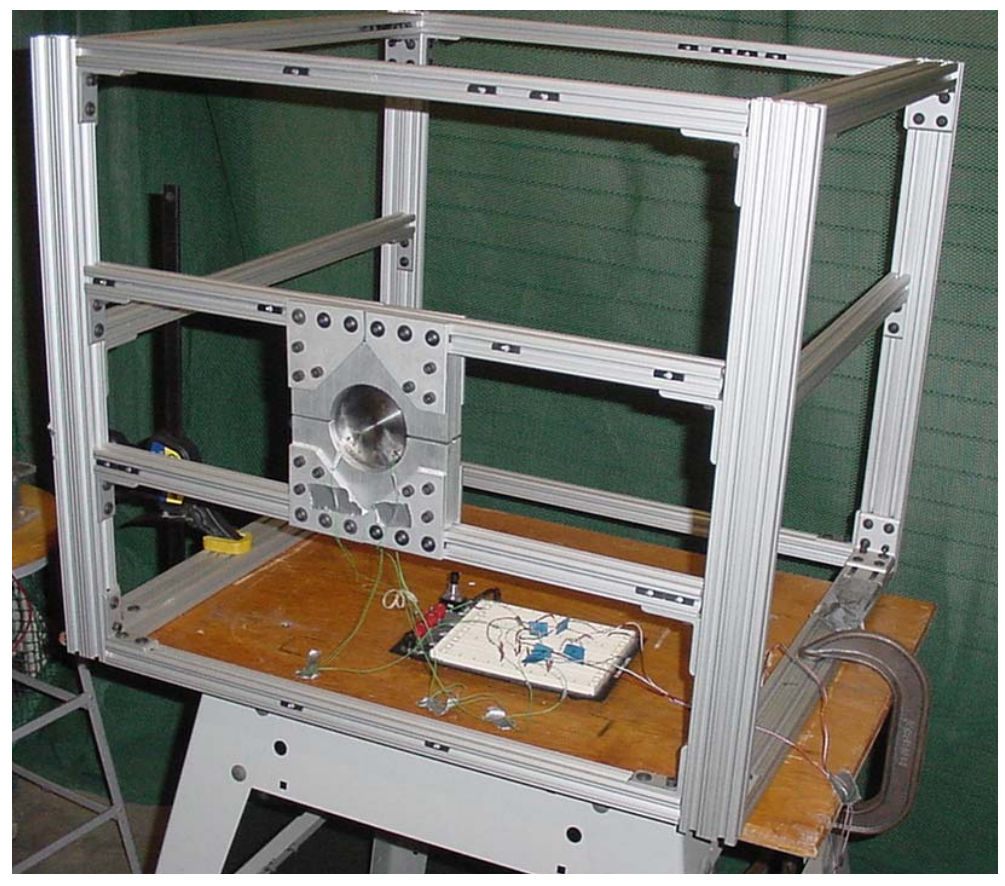

Figure 15: Titanium plate support structure using T-slot extrusions

The interior of the plate was lined with three inch sound absorbing foam (Part \#9710T46 from McMaster). The foam has a 0.96 Noise Reduction Coefficient (NRC). This sound absorbing foam helped diminish any unwanted background noise from within the lab as well as reverberation. Lining the plate structure with acoustic foam also will make simulation easier, because it allows the computer model to have a non-reflective boundary condition. With the foam, the simulation can be treated as an infinite domain, allowing us to use the acoustic BEM solver. In Figure 16, the backside of the plate fixture can be seen along with the acoustic foam. 


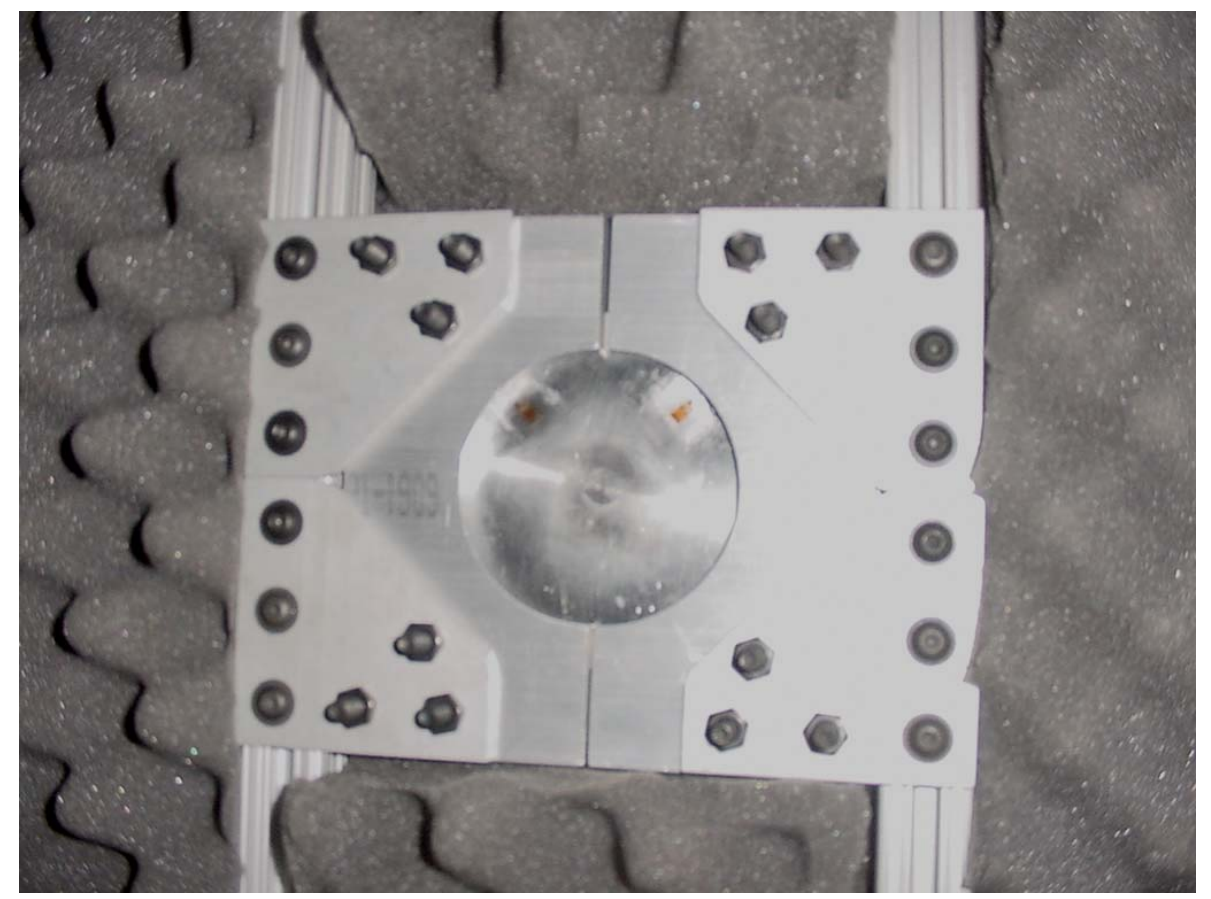

Figure 16: Titanium plate secured into the plate fixture using mounting brackets.

The microphone used to gather acoustical data was a G.R.A.S. prepolarized microphone (type 40AE) paired with a G.R.A.S. preamplifier (type 26CA). This type of microphone is favored because it does not need an external source of polarization voltage. The microphone can cover frequency ranges from $3.15 \mathrm{~Hz}$ to $20 \mathrm{kHz}$. The assembled microphone can be seen in Figure 17.

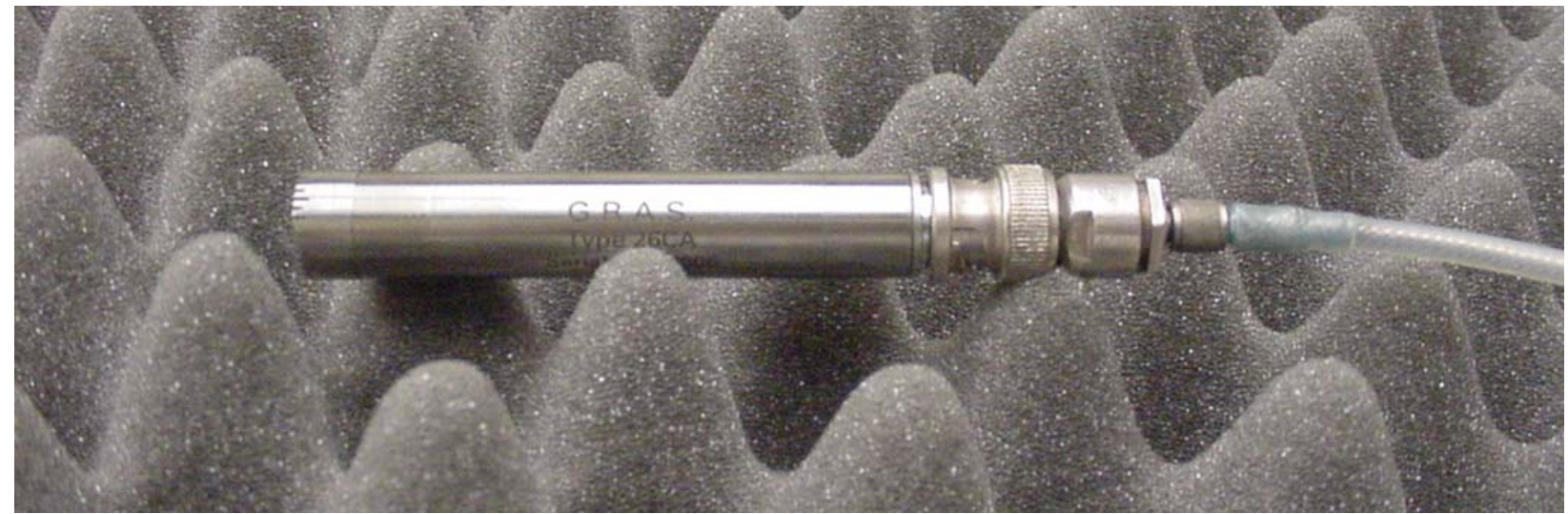

Figure 17: G.R.A.S. 1/2" microphone 
The microphone was attached to a constant current power supply then to the back of the LDS analyzer as shown in Figure 18.

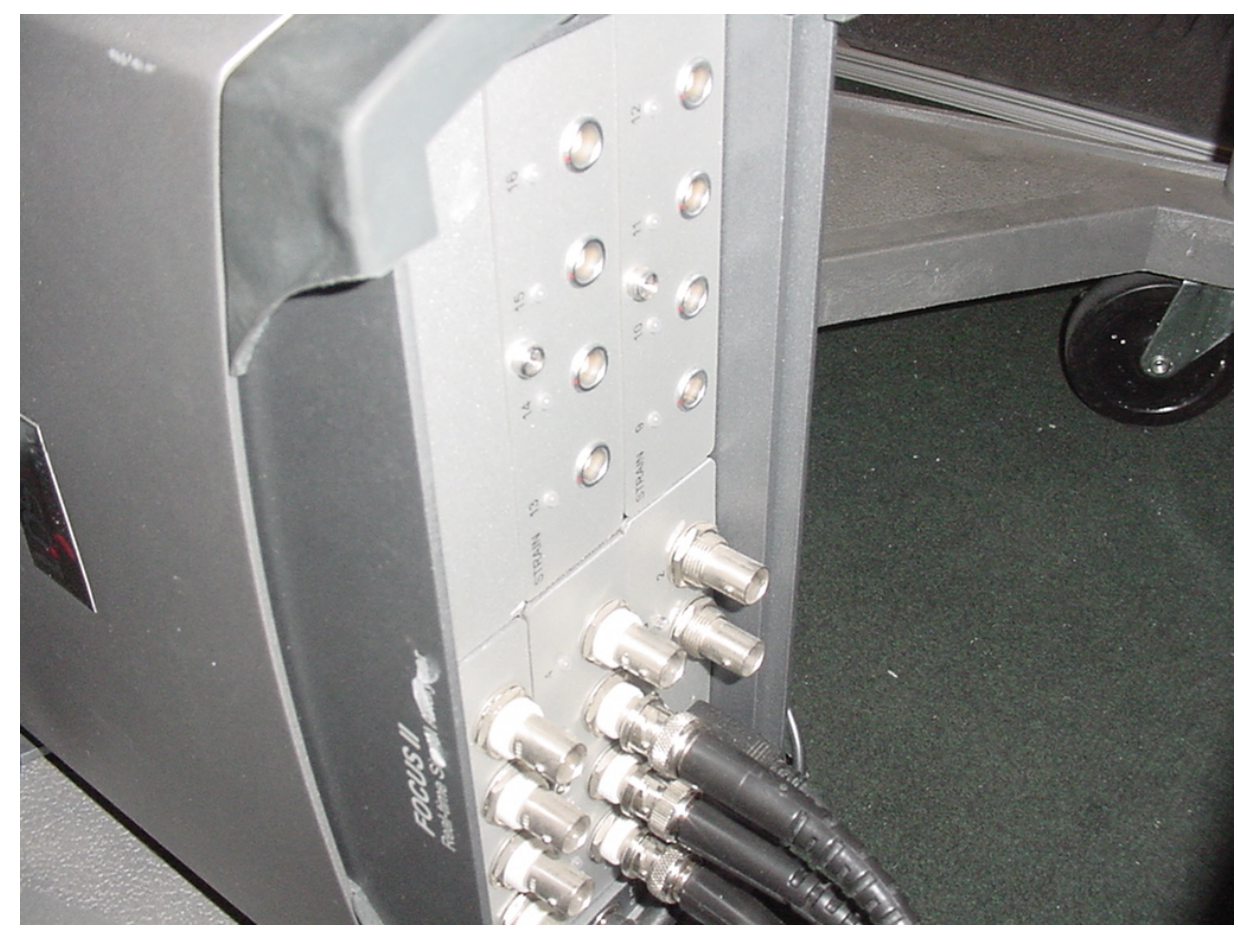

Figure 18: Inputs plugged into the back of the LDS signal analyzer.

The LDS analyzer is a multichannel multi-purpose data acquisition system. It houses the necessary inputs for both acoustic and strain data collection. It connects via a USB cable to a laptop on a portable cart. Using the PRO FOCUS II software on the computer, all the data was recorded in real time using high accuracy FFT analyzers.

\section{III.2 Computer Simulation of the Titanium Plate}

This subsection presents the FEA model and a plate mesh refinement check, followed by the golf ball model. With the plate secured in the anechoic fixture, acoustic and strain data was taken to compare to the simulation modes from a modal analysis. Finally, the BEM simulation is described. Results from this are given in the next subsection. 


\section{III.2.1 Computer Modeling of Plate}

The simulation went through three different steps in characterizing the system. If a model seems to be too stiff, the problem would most likely be caused from the boundary conditions not accurately modeling the how the structure is mounted and a new revision must be created. The first model was a simple circular plate. This was used for the mesh convergence of the plate. The actual geometry of Titleist's plate made the task more difficult because through the thickness of the plate is not uniform, one edge is smooth while the other has a drastic increase in thickness shown in Figure 19.

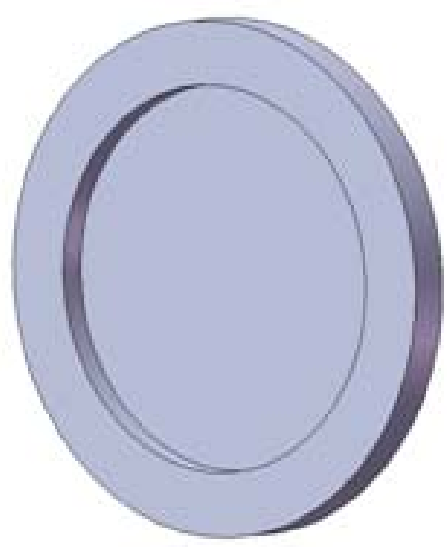

Figure 19: SolidWorks model of the titanium plate highlighting the thickness variations.

To make sure the mesh created by TrueGrid ${ }^{\circledR}$ was acceptable, a mesh convergence graph was created with different mesh densities. The plate was created with a scale factor in front of the indices for the mesh to make mesh convergence simpler. Different meshes were created by simply changing the scale factor from one to two, two to three, etc. Meshes were characterized by their scale factor (i.e. Mesh No. 1, Mesh No. 2, and so on). A second convergence criteria was determined from the actual plate by loading the plate with a load cell and measuring strain 
with the data acquisition system. Mesh convergence was determined by varying meshes with increasing density and comparing to actual data gathered [Volkoff-Shoemaker]. The load curve and boundary conditions of the test are shown in Figures 20 and 21 respectively.

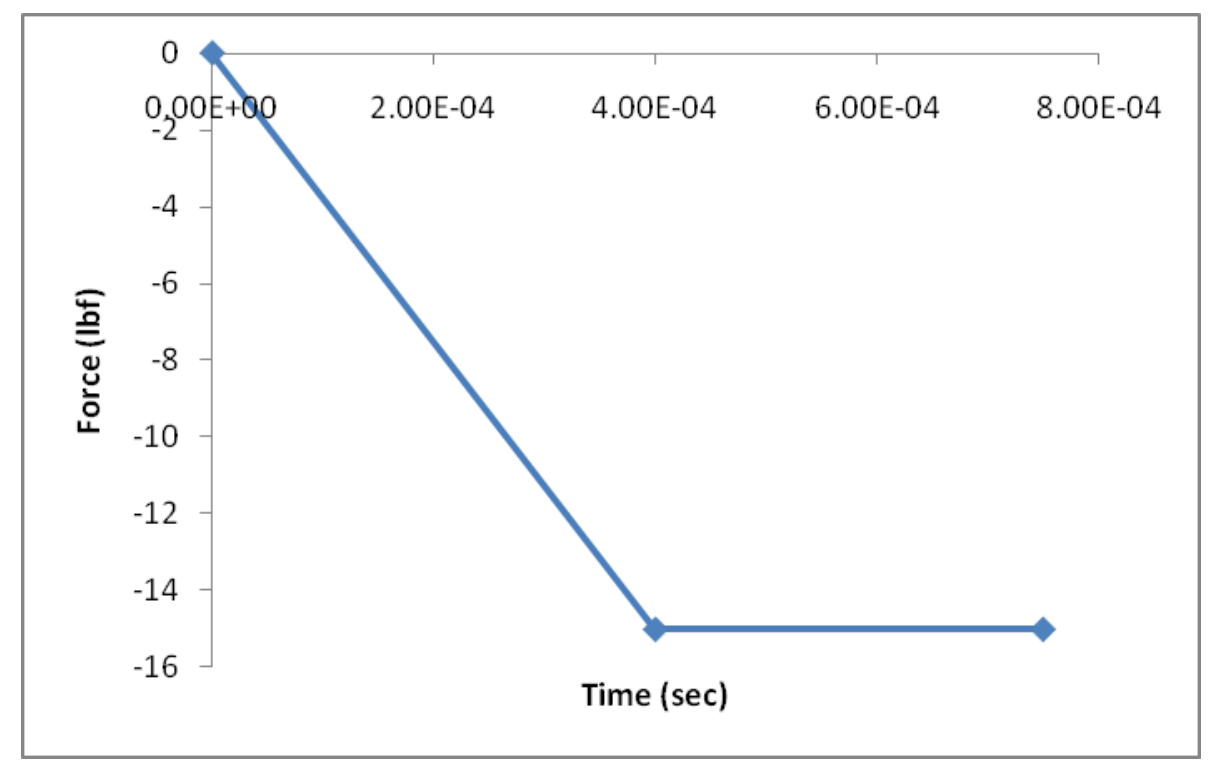

Figure 20: Load curve used for mesh convergence.

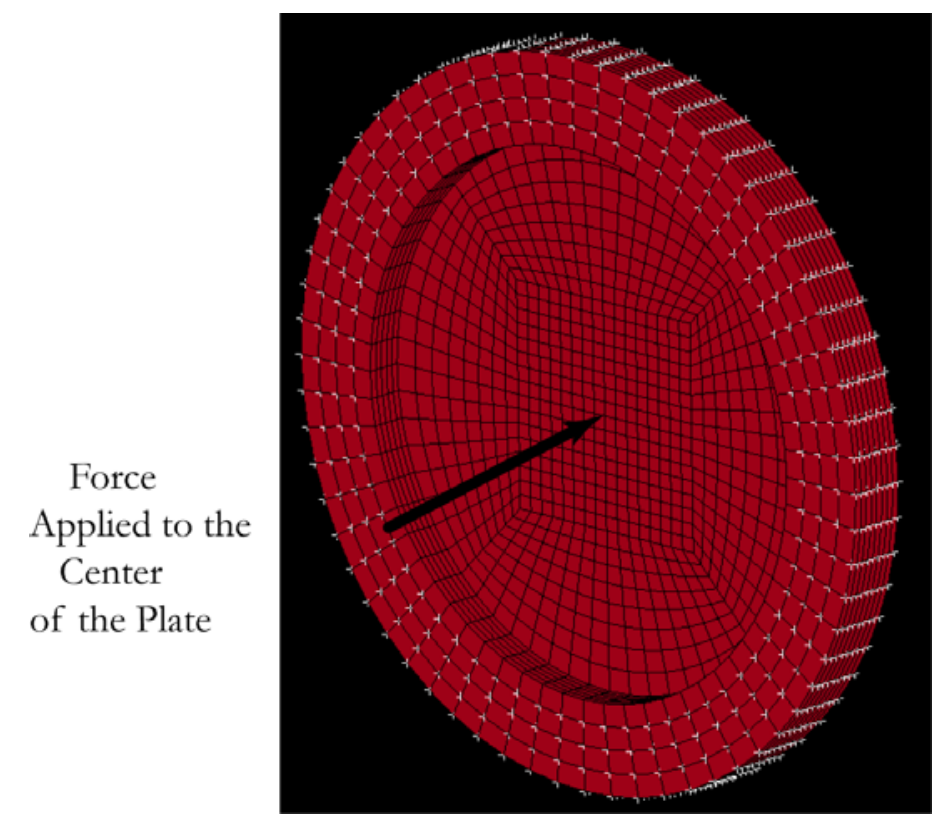

Figure 21: Boundary conditions of mesh convergence. 
The convergence plot, shown in Figure 22, demonstrates that the strain at the center of the plate reaches a fairly steady state value. There is some variation, and that comes from the scale factor not placing the node in exactly the same spot every time. Increasing the number of elements would give us a more correct answer, but the BEM is very computationally intense and less elements the faster our simulation will run.

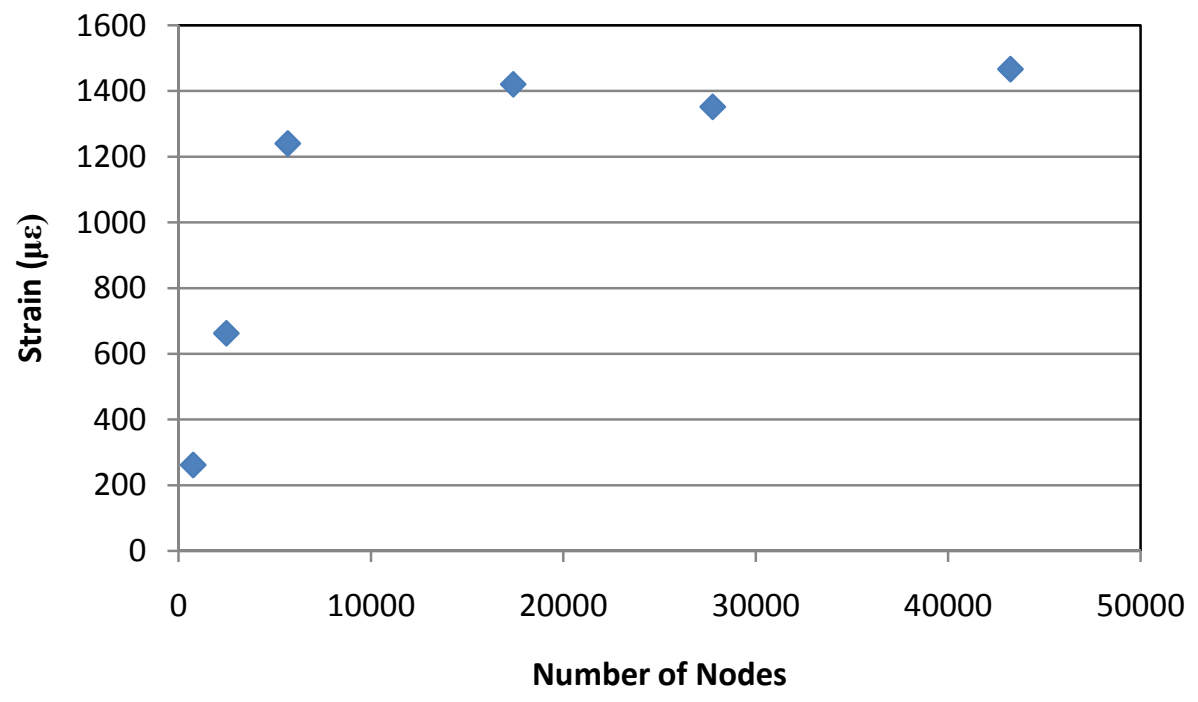

Figure 22: Mesh convergence for the plate.

To make sure the size of the elements was correct for the model, a separate mesh convergence for the ball and plate was run. The second mesh convergence was a simple approximation of the ball impacting a plate and can be seen in Figure 23. The outer edges of the plate were fixed and the ball was fired at the center of the plate. The TrueGrid $\AA$ input file was created so that the scale factor would produce the same element size on the plate and the ball. The resulting mesh convergence plot can be seen in Figure 24. 


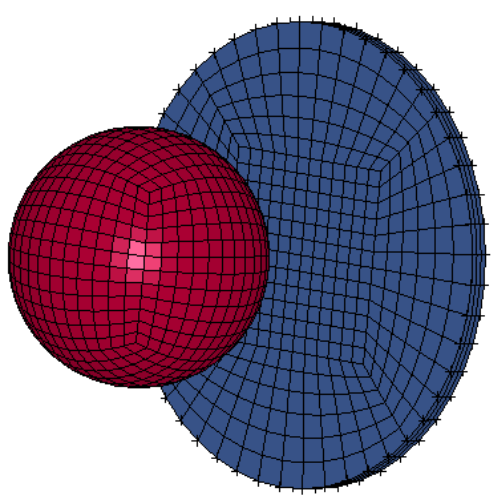

$\mathfrak{L}_{x}^{Y}$

Figure 23: Setup for ball and plate mesh convergence with fixed boundary conditions.

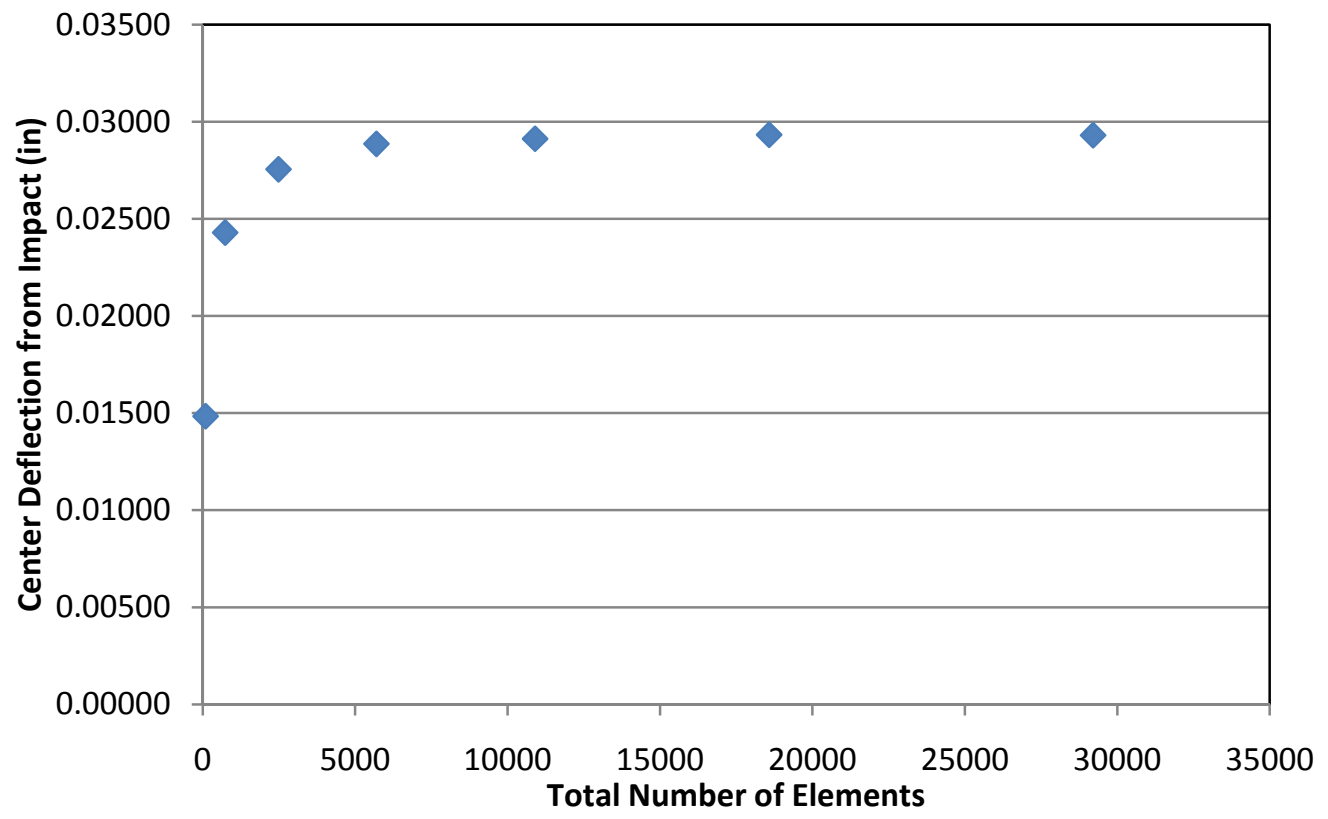

Figure 24: Mesh convergence for the ball and plate.

Mesh 5 was the best choice as seen from both mesh convergence plots. The results in Table 2 show that the center deflection hit a steady state value with less than a $1 \%$ change between 
meshes. The element size determined from the mesh convergence graph was applied to all the support structures that were modeled. Eventually all the different parts of the plate support structure were modeled due to conformance issues.

Table 2: Mesh convergence data from the ball and plate.

\begin{tabular}{|ccccc|}
\hline Mesh & $\begin{array}{c}\text { No. } \\
\text { Elements }\end{array}$ & No. Nodes & $\begin{array}{c}\text { Max Deflection } \\
\text { (in) }\end{array}$ & \% Diff \\
\hline $\mathbf{1}$ & 92 & 139 & 0.01483 & $\mathrm{n} / \mathrm{a}$ \\
\hline $\mathbf{2}$ & 736 & 884 & 0.02429 & $63.8 \%$ \\
\hline $\mathbf{3}$ & 2484 & 2789 & 0.02755 & $13.4 \%$ \\
\hline $\mathbf{4}$ & 5696 & 6197 & 0.02886 & $4.8 \%$ \\
\hline $\mathbf{5}$ & 10900 & 11645 & 0.02912 & $0.9 \%$ \\
\hline $\mathbf{6}$ & 18576 & 19613 & 0.02933 & $0.7 \%$ \\
\hline $\mathbf{7}$ & 29204 & 30581 & 0.02930 & $0.1 \%$ \\
\hline
\end{tabular}




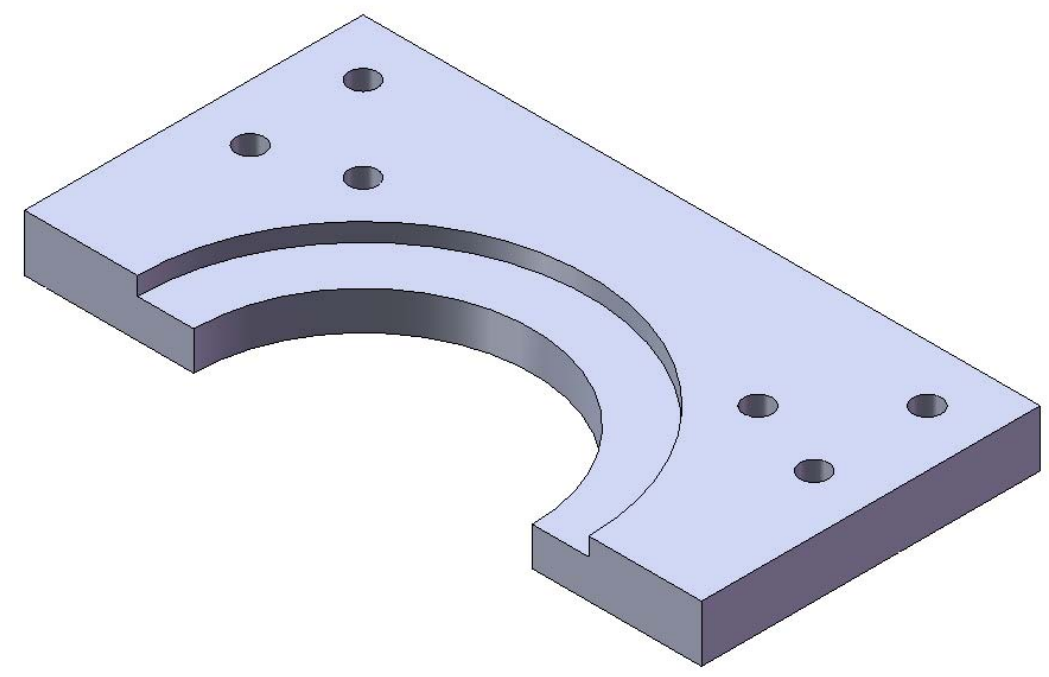

Figure 25: SolidWorks model of the machined support brackets for the titanium plate.

The plate support brackets were simple and easy to mesh because it was made of solid aluminum in Figure 25. The part was defeatured and made so the nodes of the outer rim of the plate would match with inner nodes of the bracket. The supporting beams, on the other hand, were more difficult. The beams were T-slot extrusions which are very difficult to model if the geometry is matched exactly. Drawings for the cross-section can be found in Appendix A. As an approximation, an equivalent beam with a modified Young's modulus was determined from Eq 15.

$$
E I_{T-\text { slot extrusion }}=E I_{\text {beam model }}
$$

The second most important part to model was the golf ball. In all our experimentation a Titleist ProV1 performance golf ball was used. This ball is made of three components: core, mantle, and cover. Looking at Figure 26 one can see the different materials in the cross-section of the golf ball. 


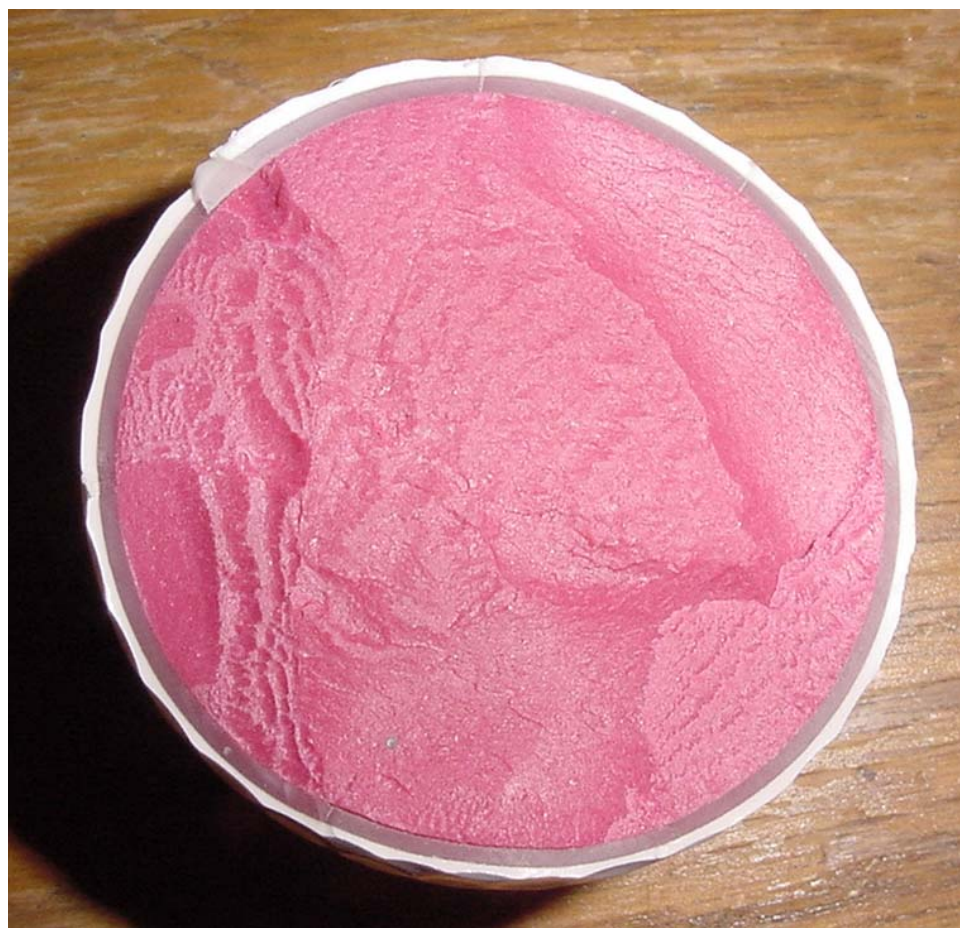

Figure 26: Titleist ProV1 golf ball cut in half showing the core, mantle, and cover thicknesses.

In Tanaka’s paper, a Mooney-Rivlin material did not fully characterize the deformation undergone at impact [Tanaka]. His findings showed that for a three piece ball, its best to include the viscoelastic effects on the behavior of the golf ball [Tanaka]. The reason being that the viscoelastic effects have a large influence on the maximum deformation, deformation histories during unloading, and the rebounding velocities. 
Table 3: Golf ball material properties provided by Tanaka.

\begin{tabular}{|llll|} 
& Core & Mantle & Cover \\
\hline $\mathbf{E}_{\mathbf{0}}(\mathbf{M P a})$ & 50 & 25 & 400 \\
\hline $\mathbf{v}$ & 0.49 & 0.49 & 0.45 \\
\hline $\mathbf{G}_{\mathbf{p}}$ & 0.4 & 0.4 & - \\
\hline $\boldsymbol{\tau}(\mathbf{m s})$ & 0.04 & 0.04 & - \\
\hline $\boldsymbol{\rho}\left(\mathbf{k g} / \mathbf{m}^{\mathbf{3}}\right)$ & 1150 & 1150 & 950 \\
\hline
\end{tabular}

Using material properties from Tanaka's paper found in Table 3, a finite element model of the golf ball was created. The viscoelastic material model used in Tanaka's paper utilized the Prony series parameters $\mathrm{g}$ and $\tau$ seen in $\mathrm{Eq}(16)$.

$$
G \tau=G_{0}\left[1-g_{1}^{\rho}\left\{1-\exp \left(-t \tau_{1}^{G}\right)\right\}\right]
$$

The resulting LS-DYNA ${ }^{\circledR}$ coefficients determined from Tanaka's work that were used in the Prony series approximation can be found in Table 4 and Table 5.

Table 4: Derived coefficients for shear moduli $G_{i}$ and decay constants $\beta_{i}$ in the Prony series material model used in LS-DYNA®.

\begin{tabular}{|ccc|}
\hline & $\mathrm{G}_{\mathrm{i}}(\mathrm{psi})$ & $\beta_{\mathrm{i}}(\mathbf{1} / \mathrm{sec})$ \\
\hline Core Term 1 & 1450.0 & 0 \\
\hline Core Term 2 & 966.7 & 25000 \\
\hline Mantle Term 1 & 725.0 & 0 \\
\hline Mantle Term 2 & 483.3 & 25000 \\
\hline
\end{tabular}


Table 5: Curve fitting coefficients used in the Prony series.

\begin{tabular}{|ccc|}
\hline & C10 & C01 \\
\hline Core & 1184 & 24.2 \\
\hline Mantle & 592 & 12.1 \\
\hline Cover & 9475 & 193.4 \\
\hline
\end{tabular}

Tanaka provided a load history plot of the impact force of the ball hitting a rigid surface in his paper and can be seen in Figure 27a. To recreate his results, our ball model will need to closely match his findings. Tanaka's model had a viscoelastic golf ball hitting a rigid plate and our previous mesh convergence had a golf ball hitting an elastic plate. From the mesh convergence model, the plate material was switched to rigid and the resulting force history graph was produced in Figure 27b.
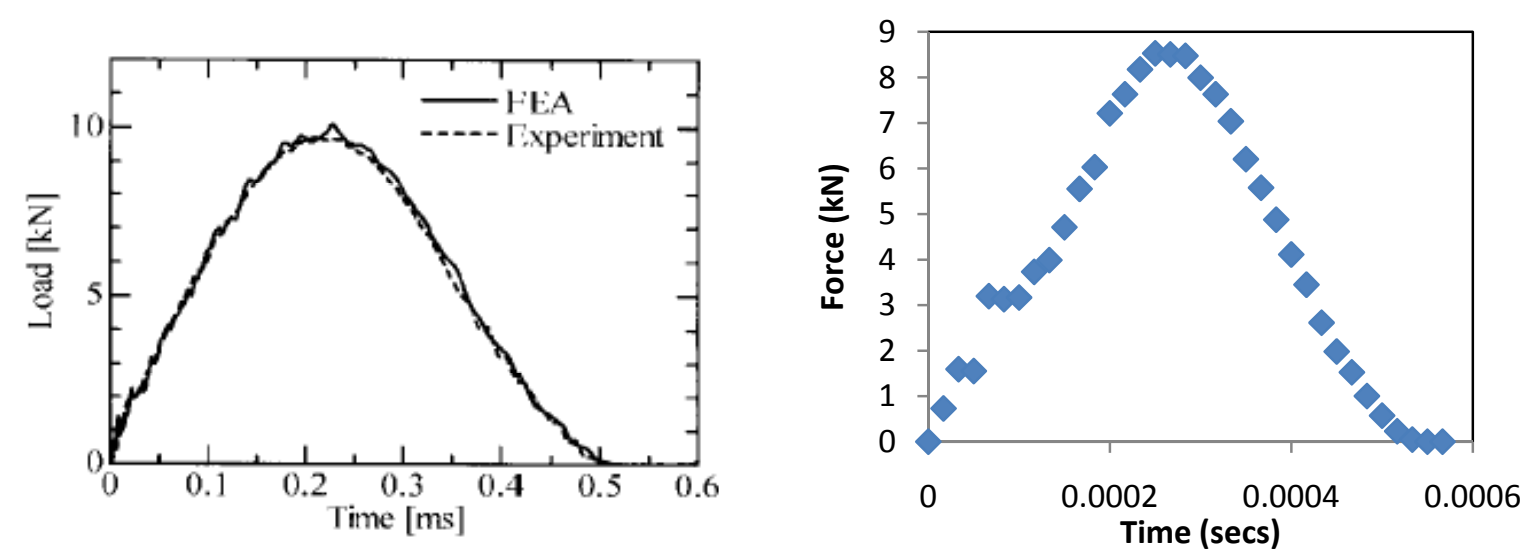

Figure 27: Tanaka's force and contact time plot (a) compared to the FE model (b) used for golf ball mesh convergence.

From previous mesh convergence tests, an adequate mesh was already determined. The three parts of the golf ball were all incorporated into the model according to Tanaka. The resulting mesh can be seen in Figure 28. 


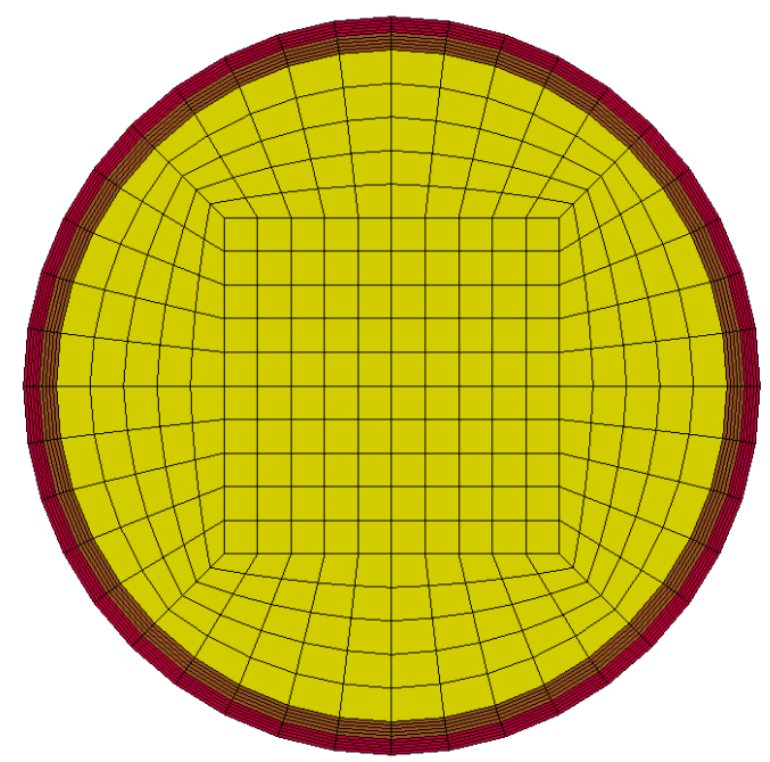

Figure 28: Cross section of the meshed golf ball showing the different materials used for the core, mantle, and cover using mesh density No. 5.

To increase the fidelity of the simulation, different models were created with varying levels of complexity. These included modeling the actual plate, the plate's support brackets, and the beams supporting the plate. All the different models were run using LS-DYNA®'s implicit eigenvalue solver and compared against actual modal analysis data.

The final most complex version included the cross-bracing bars supporting the aluminum mounts and the titanium plate. In total, six different materials were used and are shown in Figure 29. The contact definition was for the cover of the golf ball and the titanium plate. 

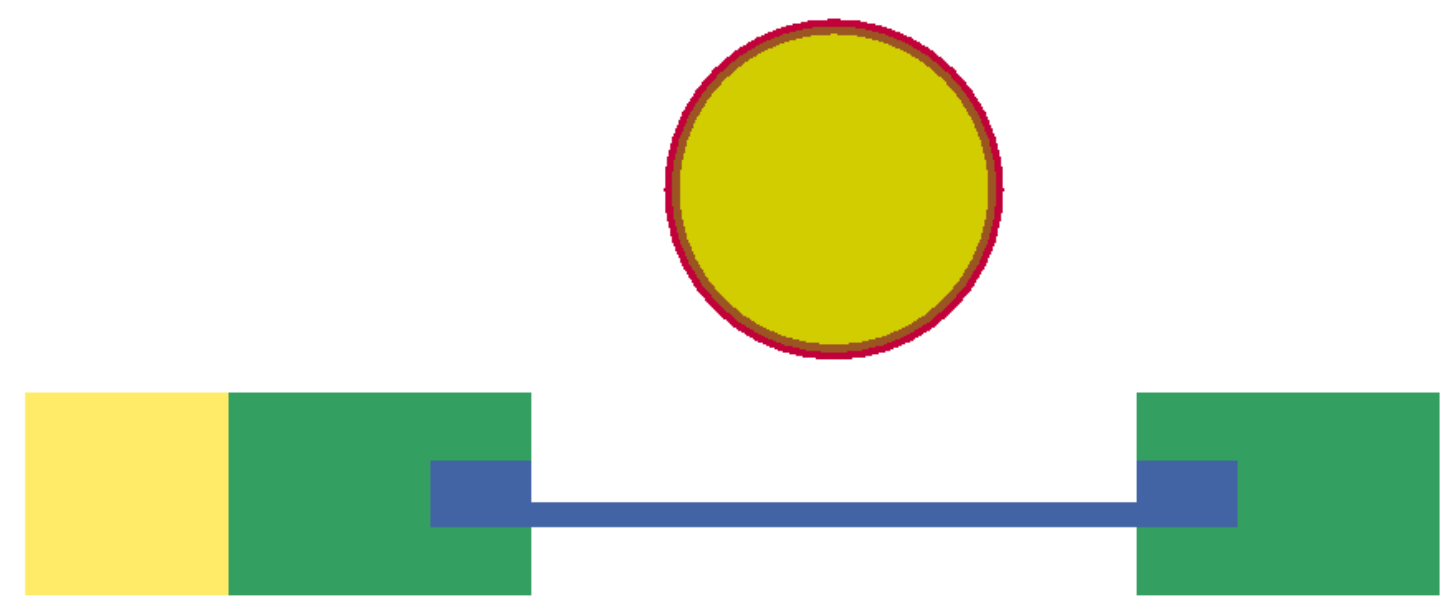

Figure 29: Cross-section of golf ball impact model.

\section{III.2.2 Modal Analysis}

A modal analysis was performed to determine what range of frequencies the titanium plate will be excited from. Now, a circular plate has been studied numerous times and the natural frequencies determined, however the unique boundary conditions of this thesis make a theoretical assumption difficult. A cross-sectional view of the Titleist plate can be found in Figure 30.

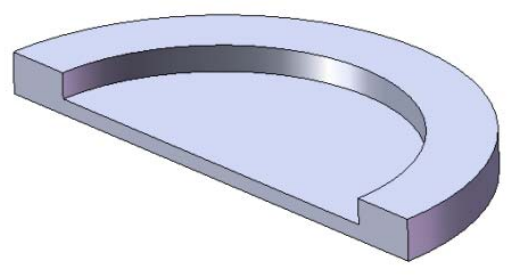

Figure 30: Titanium plate cut along the diameter showing the change in thickness. 
The model will have three different versions which will have all the possible boundary conditions: free, simply supported, and fixed on the outer edge. These findings can be compared to published results like those already derived [Blevin]. To visualize the difference between the first few natural frequency modes, the mode shapes are shown in Table 6 . The best solution is to test the plate using a force hammer and accelerometer and then compare the results to FEA. This analysis is twofold: it will help determine acoustical modes and also distinguish how well natural frequencies compare to an FFT of the sound from impact.

Table 6: Mode shapes derived by Blevin.

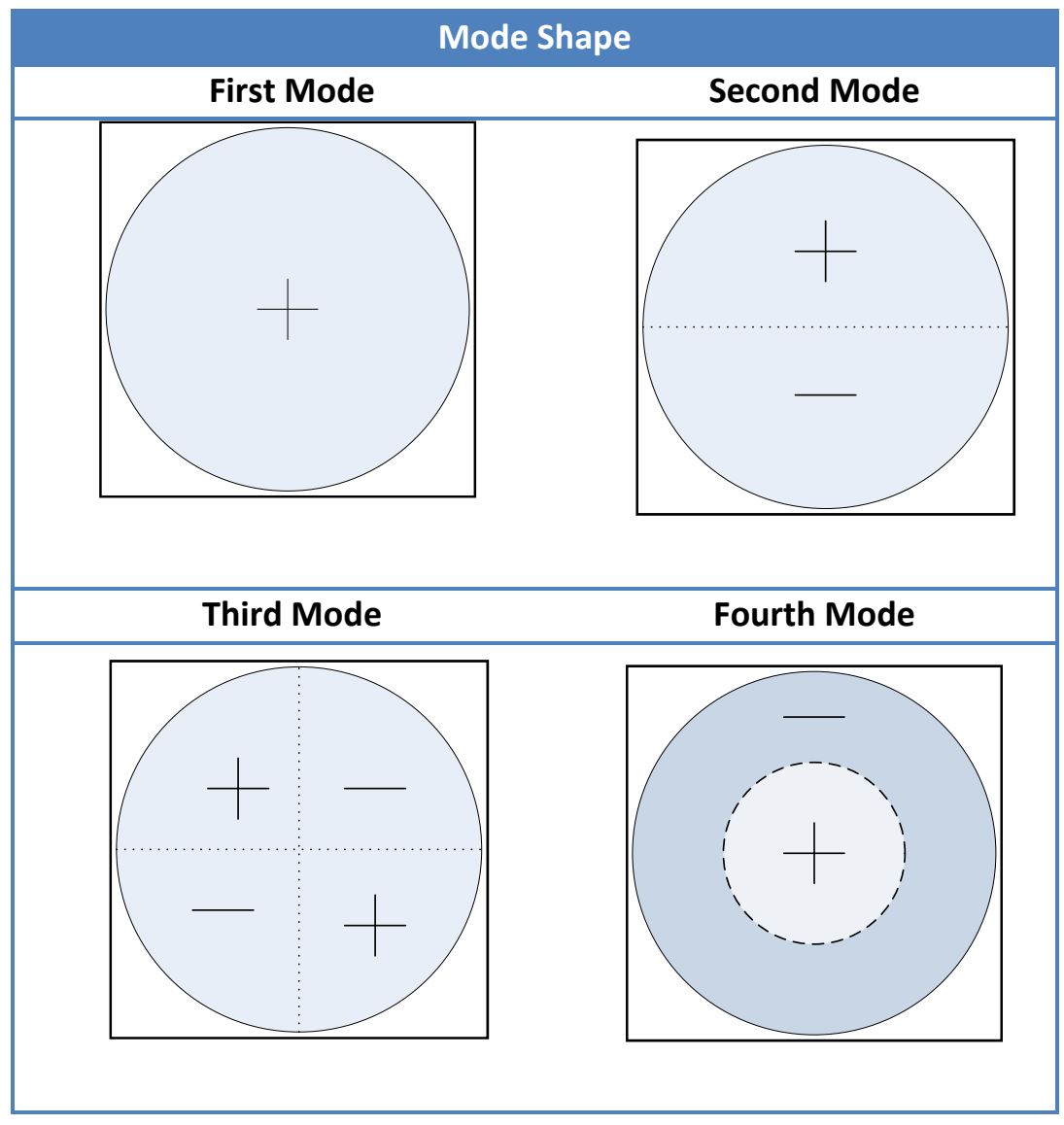

The plate was first analyzed in a free boundary condition. It was placed on a piece of sound absorbing foam to allow for rigid body modes of vibration. An accelerometer was placed at the 
center of the plate attached to an LDS signal analyzer via a microdot connection. The plate was excited from a force hammer and the consequent plot was collected with good coherence and shown in Figure 31.

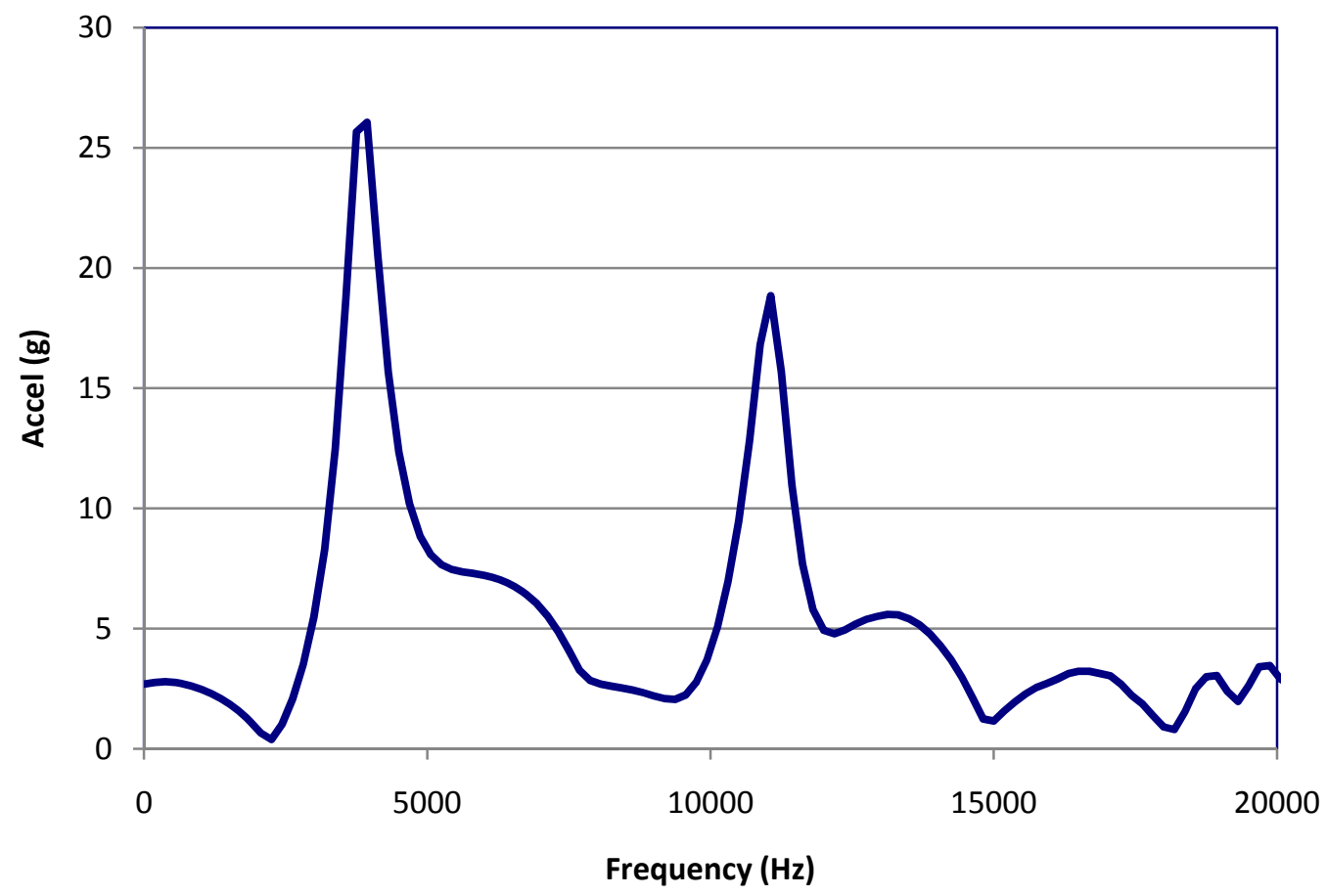

Figure 31: FFT plot of acceleration from the titanium plate excited in a free condition using a force hammer.

From the FFT data, we know that at $3940 \mathrm{~Hz}$ and $11060 \mathrm{~Hz}$ the center of the plate is easily excited. This is most likely vibrational modes that contain different number of nodal circles, the reason being that the accelerometer has only one axis that it is measuring. This makes it difficult to record mode shapes that are not along the accelerometer's axis. Therefore, the accelerometer will record the best modal data when placed in the center of a nodal circle. From here, we turn to FEA of the actual plate geometry in a free-free boundary condition as seen in Figure 32. 


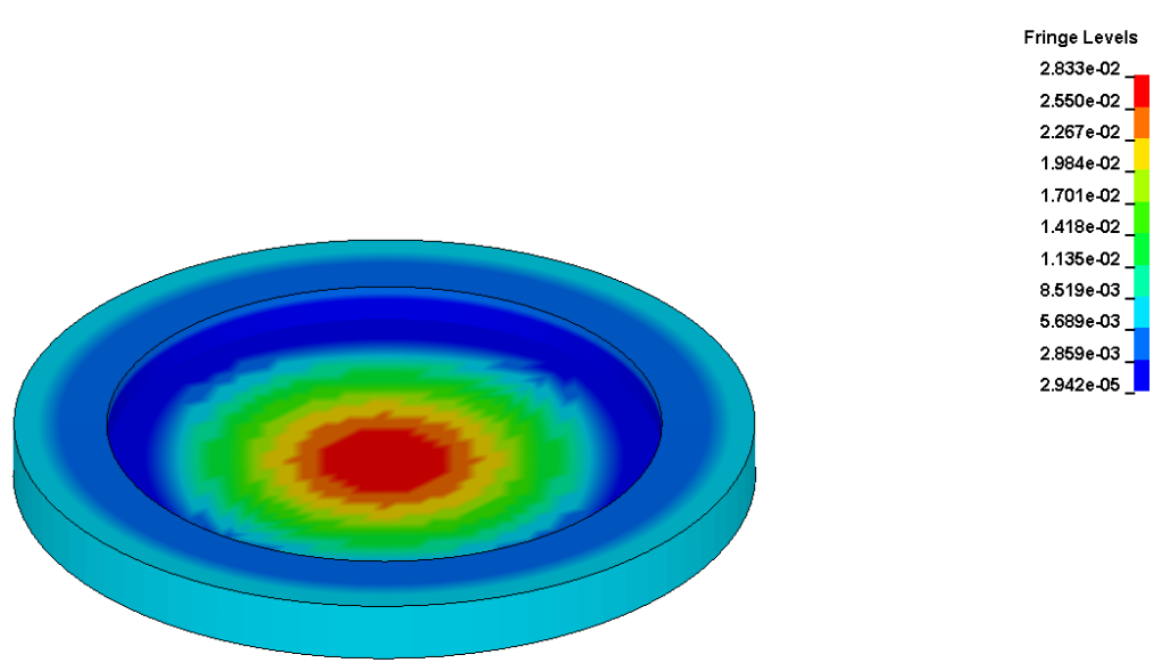

$8 x$

Figure 32: FEA result of the first modal frequency with a nodal circle.

From the modal data, we were able to tune the model to achieve better results. The elastic modulus of the titanium plate was changed until the frequencies of the FEA closely matched those measured. The result was a new elastic modulus of $14.5 \mathrm{msi}$ and it produces error of $0.5 \%$ below the first frequency and 1.1\% above the second frequency shown in Figure 33. 


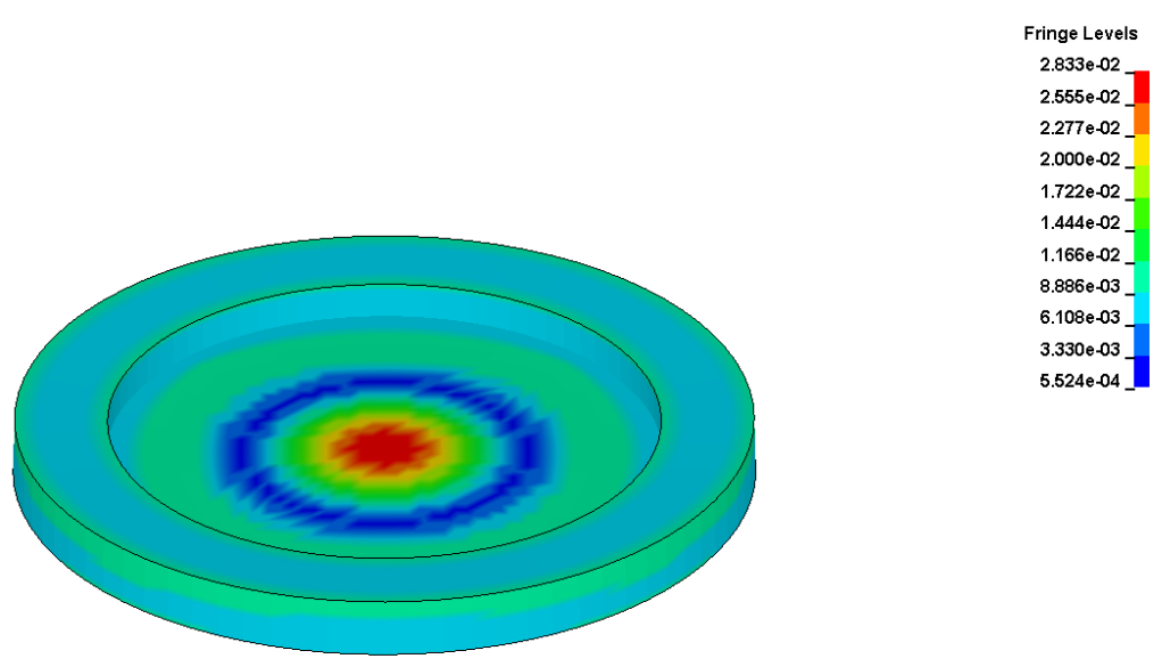

$\varepsilon_{x}$

Figure 33: FEA results of the second modal frequency with two nodal circles.

When placed in the anechoic chamber with both the accelerometer and microphone setup to take data using the LDS, the resulting FFT is as shown in Figure 34. The accelerometer was bonded to the center of the titanium plate and excited by a force hammer. The microphone was supported two inches behind the plate. All the data was gathered by the LDS analyzer at the highest possible resolution and brought into Excel for manipulation. 


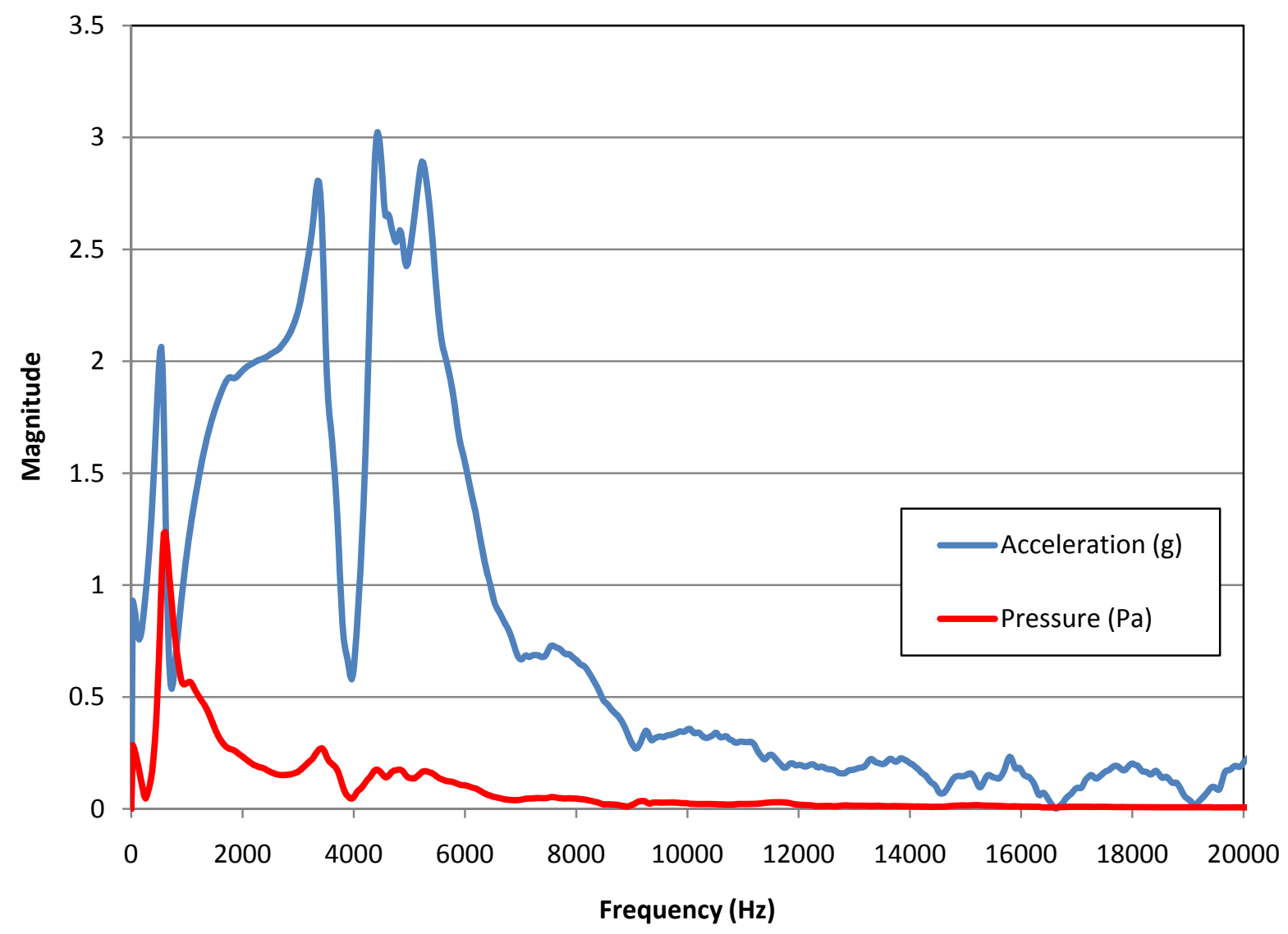

Figure 34: FFT plot of acceleration of the center plate and sound pressure

The hope was for the first natural frequency of the plate to be the main mode excited, but the result is that the first frequency is shifted to below $1000 \mathrm{~Hz}$. The peak measured frequencies can be found in Table 7. The problem came from the T-slot extrusion beams used to support the plate brackets. When compared to the plate, those beams became the main mode of deflection and for that reason, the simulation had to be changed; no longer was a simple plate model going to work. In hindsight, the supporting structure should have been ten times as stiff as the titanium plate we were measuring so it could be neglected in the simulation. 
Table 7: Peak frequencies from modal analysis.

\begin{tabular}{|rc|}
\hline Peak & \multicolumn{1}{|c|}{$\begin{array}{l}\text { Frequency } \\
(\mathrm{Hz})\end{array}$} \\
\hline $\mathbf{1}$ & 515 \\
\hline $\mathbf{2}$ & 3300 \\
\hline $\mathbf{3}$ & 4400 \\
\hline $\mathbf{4}$ & 5180 \\
\hline
\end{tabular}

From a modal analysis of the finite element model of the whole structure one sees that the $3^{\text {rd }}$ and $4^{\text {th }}$ peaks coincide with eigenfrequencies which primarily excite the titanium plate. The $4^{\text {th }}$ peak is the first mode that contains large deformation of the plate, as seen in Figure 35. This compares well with the frequencies measured by the LDS analyzer in the lab.

Freq $=\quad 5074.2$

Contours of Resultant Displacement $\min =0$, at node $\$ 9383$

$\max =1.34838$, at node\# 963

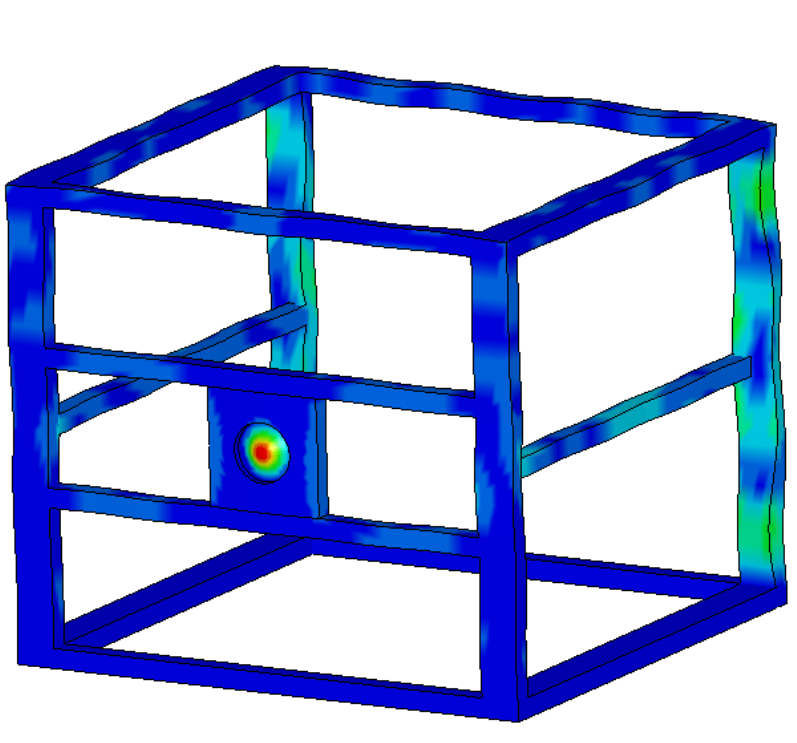

Fringe Levels

$1.348 \mathrm{e}+00$

$1.214 \mathrm{e}+00$ $1.079 \mathrm{e}+00$ $9.439 \mathrm{e}-01$ $8.090 e-01$ $6.742 \mathrm{e}-01$ $5.394 e-01$ _ $4.045 \mathrm{e}-01$ $2.697 \mathrm{e}-01$ $1.348 \mathrm{e}-01$ $0.000 \mathrm{e}+00$

Figure 35: Modal analysis of the entire structure. 
Although the data collected in the plate fixture did not coincide with what was obtained during the modal analysis of the free system, we can see from Figure 34 that the acceleration of the plate was closely related to the sound pressure levels recorded by the microphone. Recall from the flowchart highlighting the BEM solver, nodal velocities are a key factor when determining the sound pressure levels.

\section{III.2.3 BEM Solver}

From the aforementioned tests, the complexity of the model has been determined, the main mode of vibration has been confirmed, and the plate no longer is the major factor in sound production, but rather the whole structure supporting the plate is. In Figure 36, the setup for the first acoustic run can be seen. After determining the smaller model was not accurate enough, the entire structure was modeled and is seen in Figure 37.

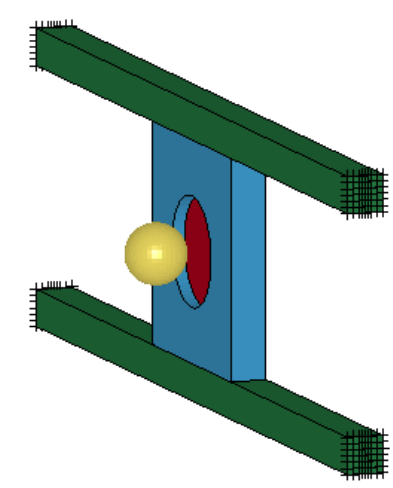

90000

Figure 36: Acoustic model of the golf ball impact showing the acoustic point in space specified by node 90000. 


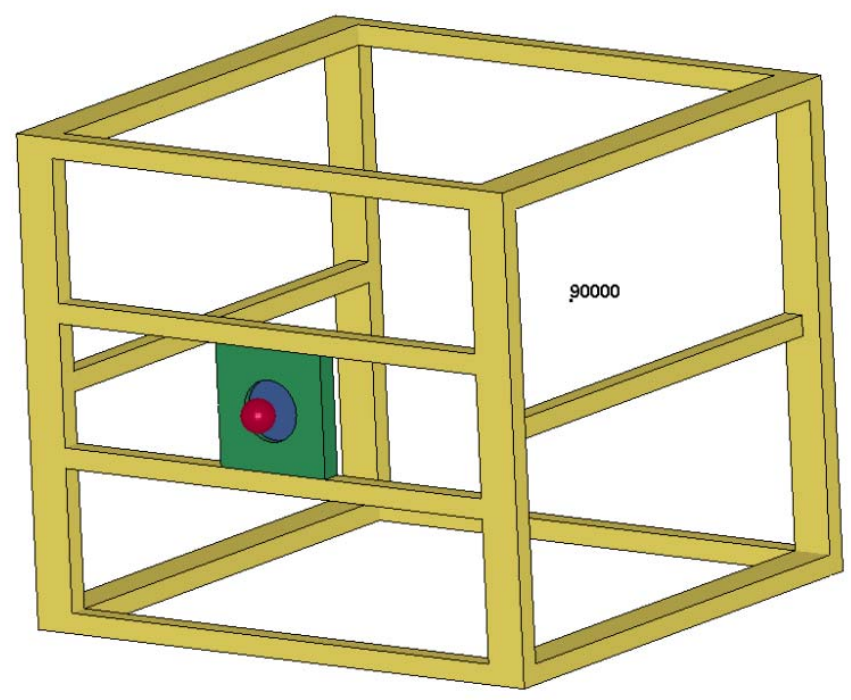

Figure 37: Acoustic model of the entire structure showing the microphone location (acoustic node 9000).

\section{III.3 Results}

The goal of this project was to produce results from a simulation that mimics real life sound. The following are the results obtained from the experiment and the LS-DYNA ${ }^{\circledR}$ simulation.

\section{III.3.1 Computer Simulation}

In LS-DYNA ${ }^{\circledR}$, users can use an embedded boundary element solver to determine the acoustic sound pressure levels. Using the boundary element method (BEM), the velocities of the vibrating structure are used to solve Helmholtz's equation. It relates the pressure wave created by the sound and the speed of the medium it is in.

From the BEM solver in LS-DYNA®, the sound theoretically measured at the microphone is shown in Figure 38. The BEM solver is a very computationally intense solver and with the total number of elements involved in this model, the computational time took one hundred hours to complete. In the case of the plate and its support brackets, Raleigh's method is appropriate 
because the components are relatively flat. While Raleigh's method is computationally more efficient, it would not give accurate results when applied to the 3D shape of a golf driver head.

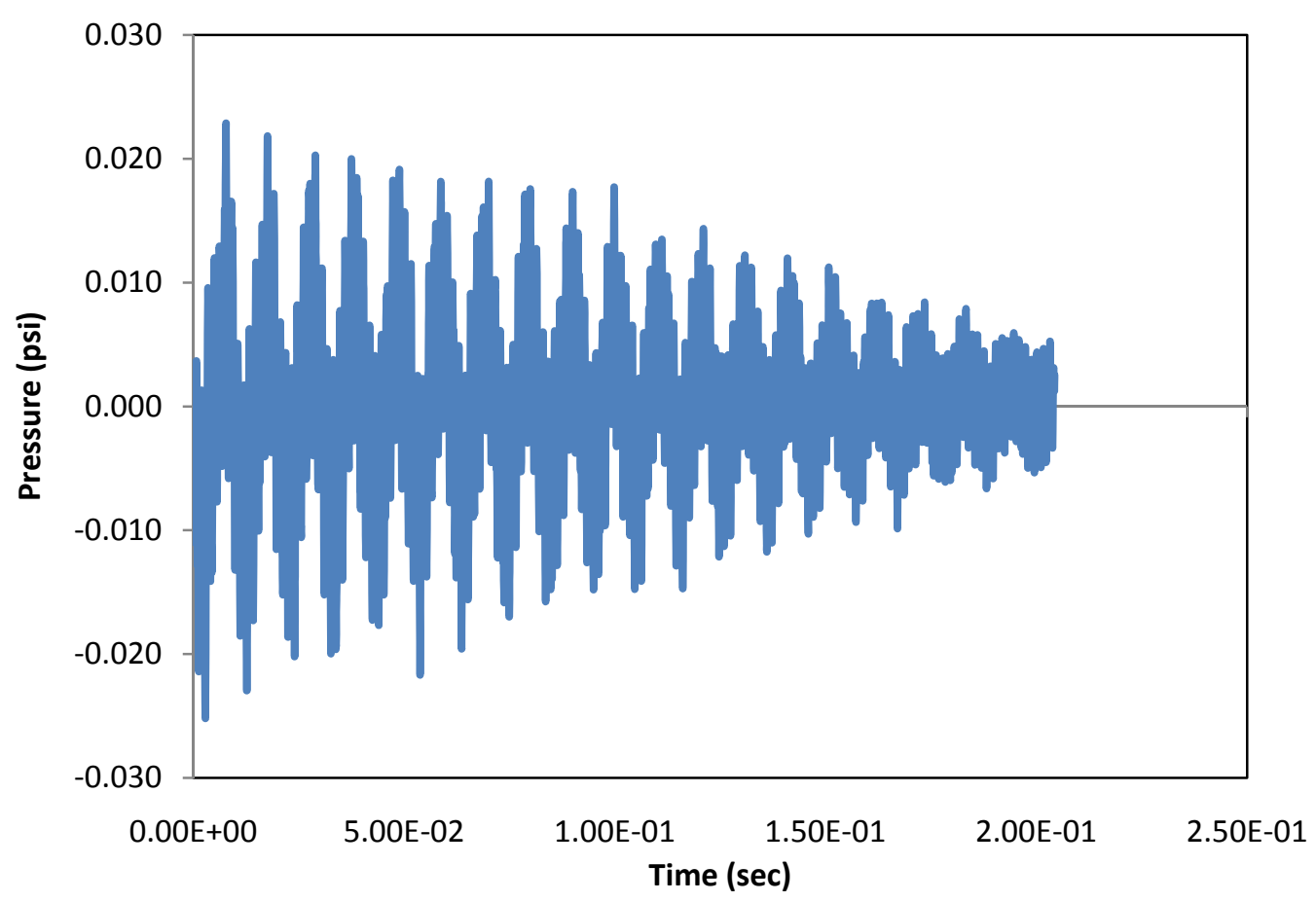

Figure 38: Sound pressure from BEM analysis.

The FFT plot of the data in Figure 39 shows distinct frequencies which coincide with eigenfrequencies determined from modal analysis. When the pressure data is brought into MATLAB ${ }^{\mathrm{TM}}$, the sound output is a short chirp. 


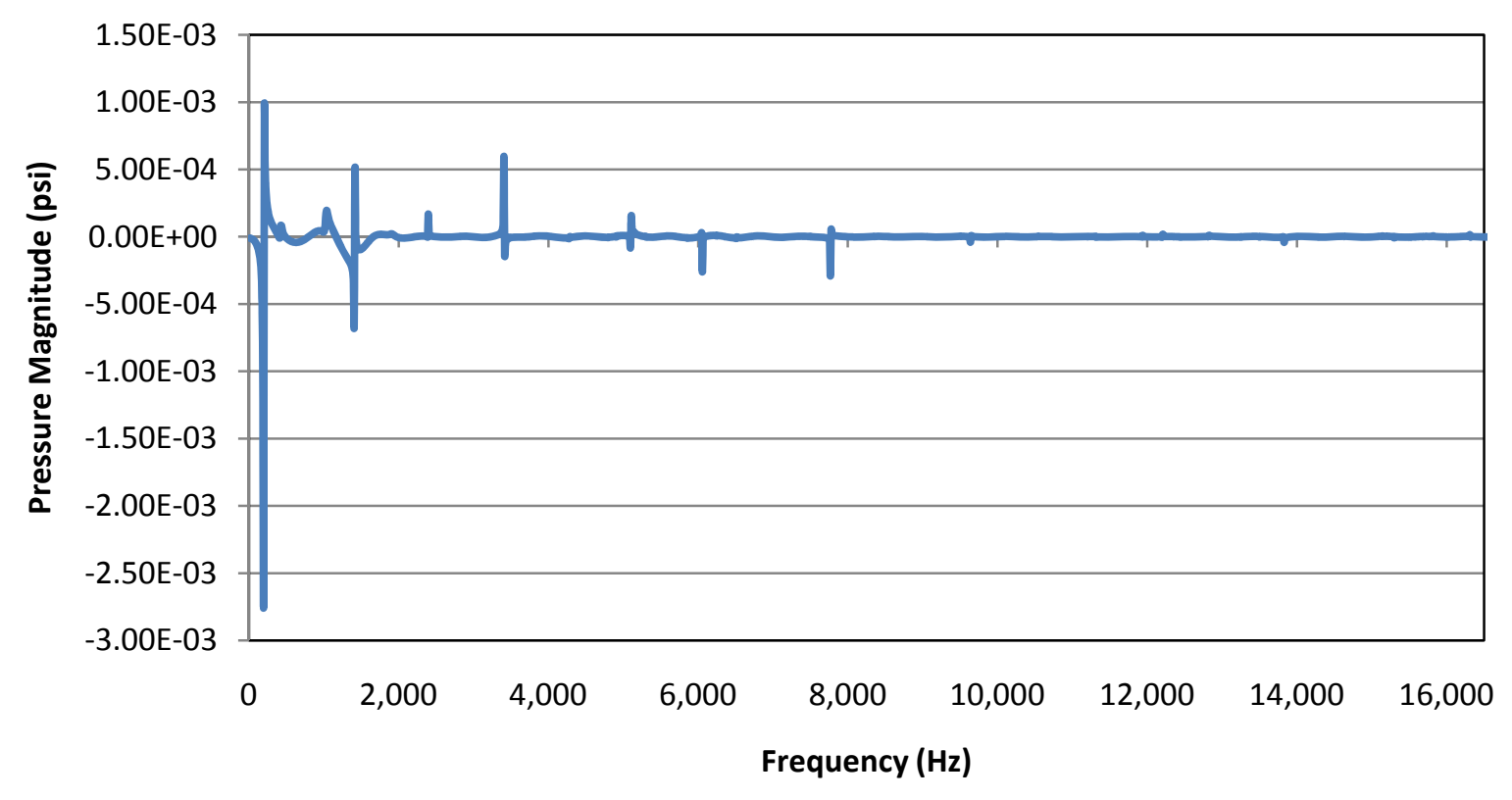

Figure 39: BEM FFT plot from plate fixture.

\section{III.3.2 Air Cannon Test}

The air cannon test was successful in gathering acoustical data; however the quality of the data collection was not the best. The anechoic chamber did not perform as hoped in keeping the exterior sounds out. In Figure 40, the air cannon was fired without a golf ball and the resulting pressure and time graph is shown. 


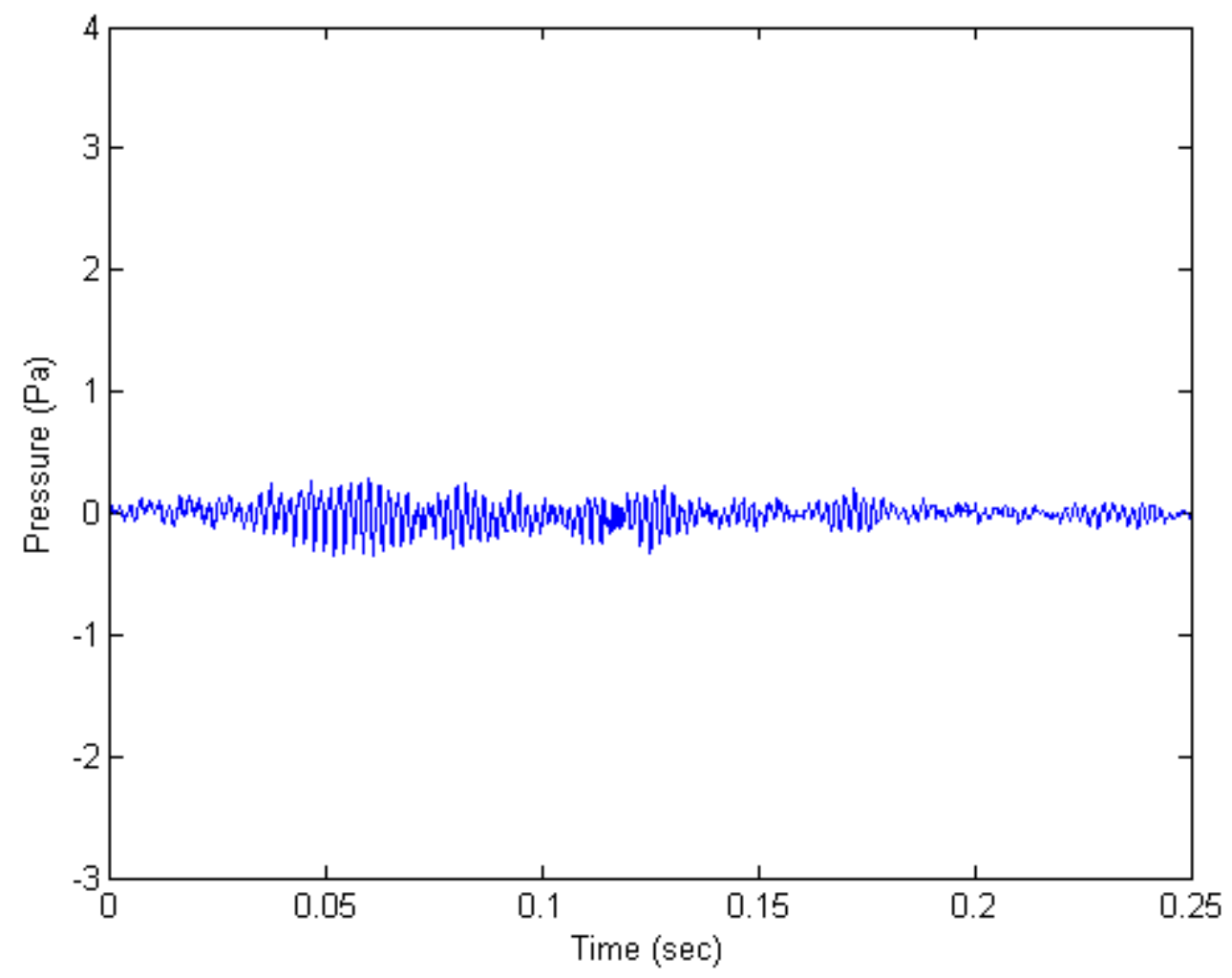

Figure 40: Microphone data from a "dryfire" of the aircannon.

To try and account for the noise created by the air cannon, signals from the actual impacts had the data from the "dryfire" subtracted from them. The result is a cleaner sound when played through the computer's speakers.

When a golf ball was loaded in the chamber of the air cannon, the resulting acoustic data is presented in Figure 41. The accompanying FFT graph of the impact is in Figure 42. 


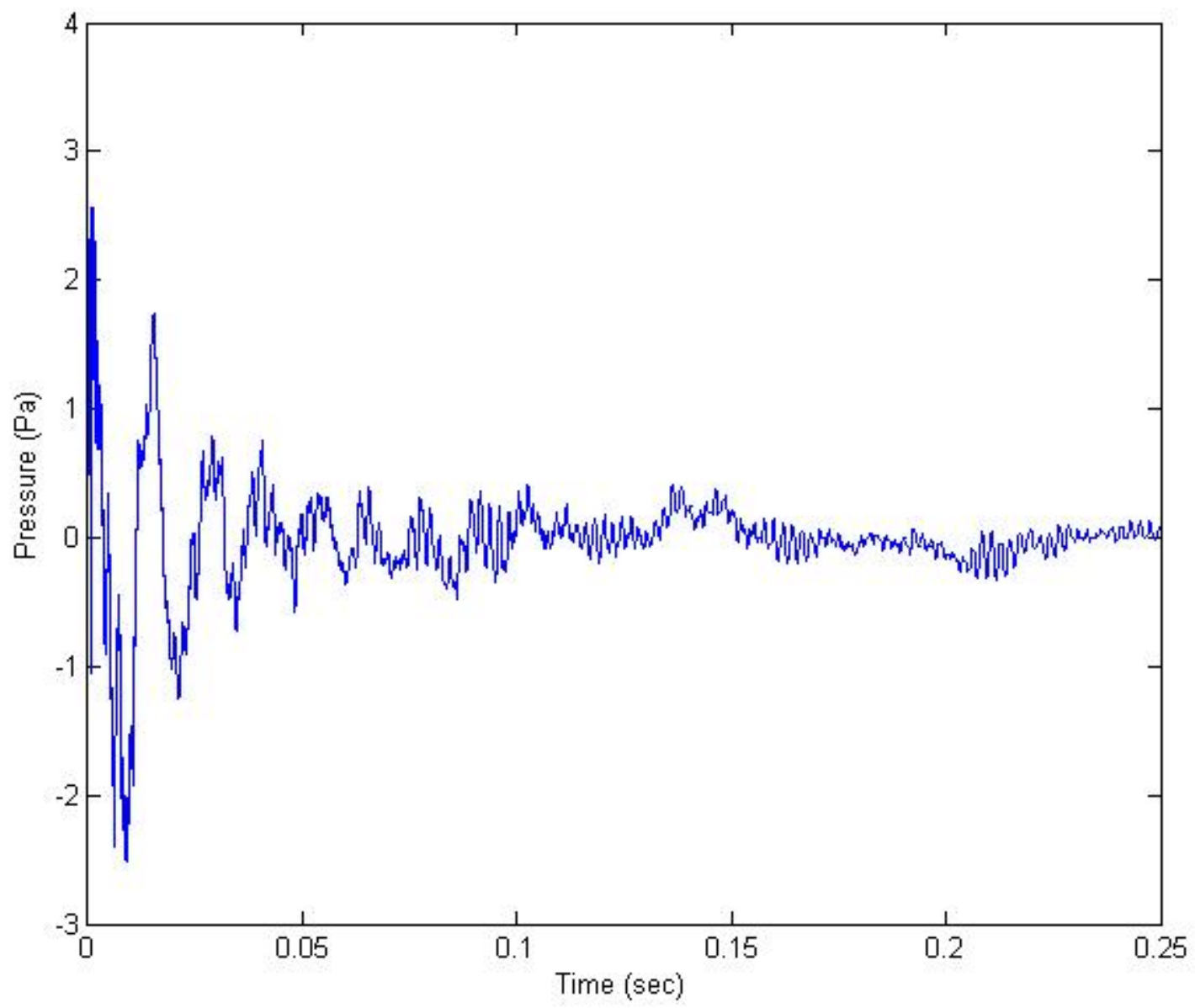

Figure 41: The pressure readings from the G.R.A.S. microphone of a 78 MPH impact. 


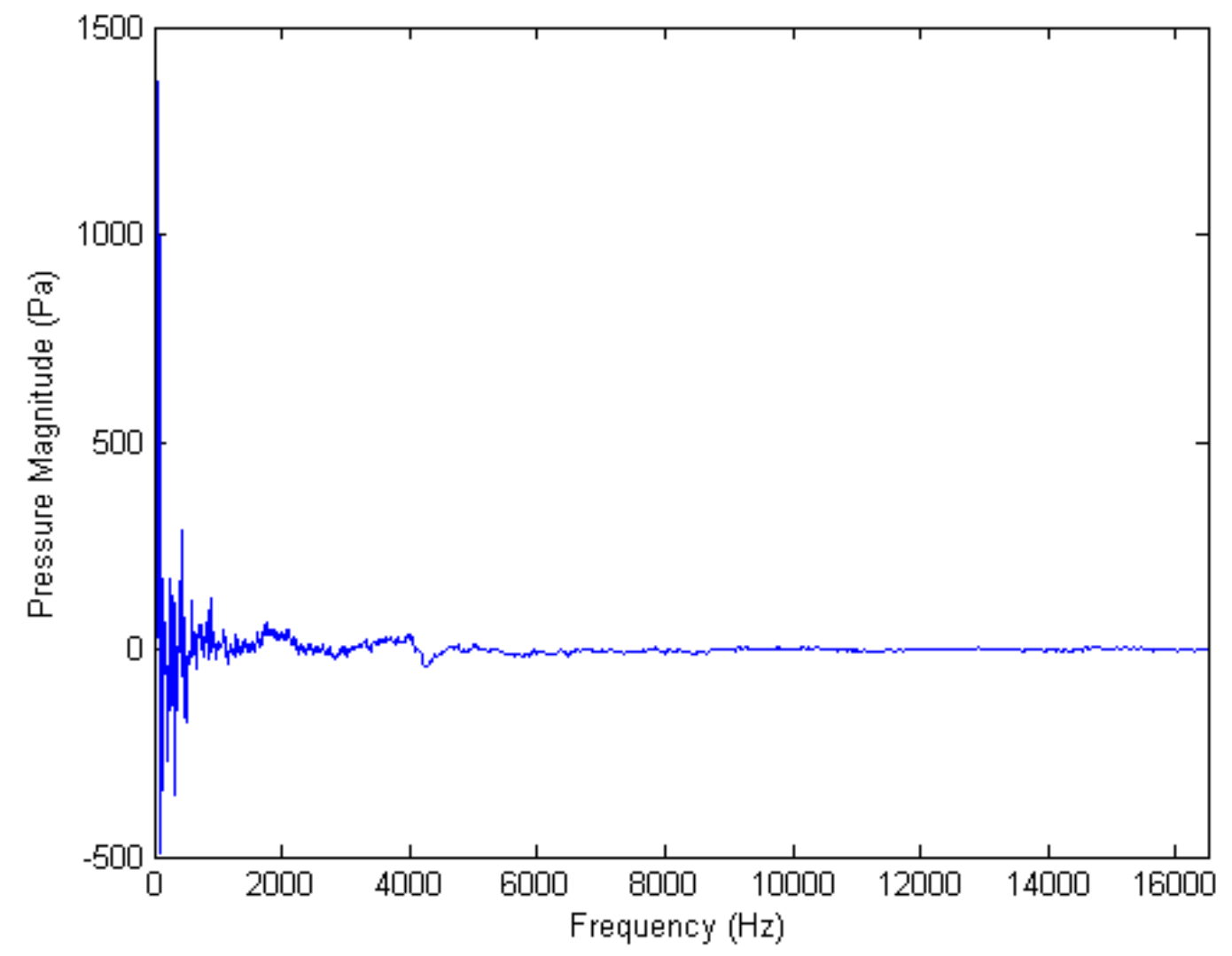

Figure 42: FFT of the pressure readings from the microphone.

It's a little difficult to see, because the majority of the sound spectrum is coming from the lower frequency range, therefore in Figure 43, the frequency range has been changed to a max of $2000 \mathrm{~Hz}$. 


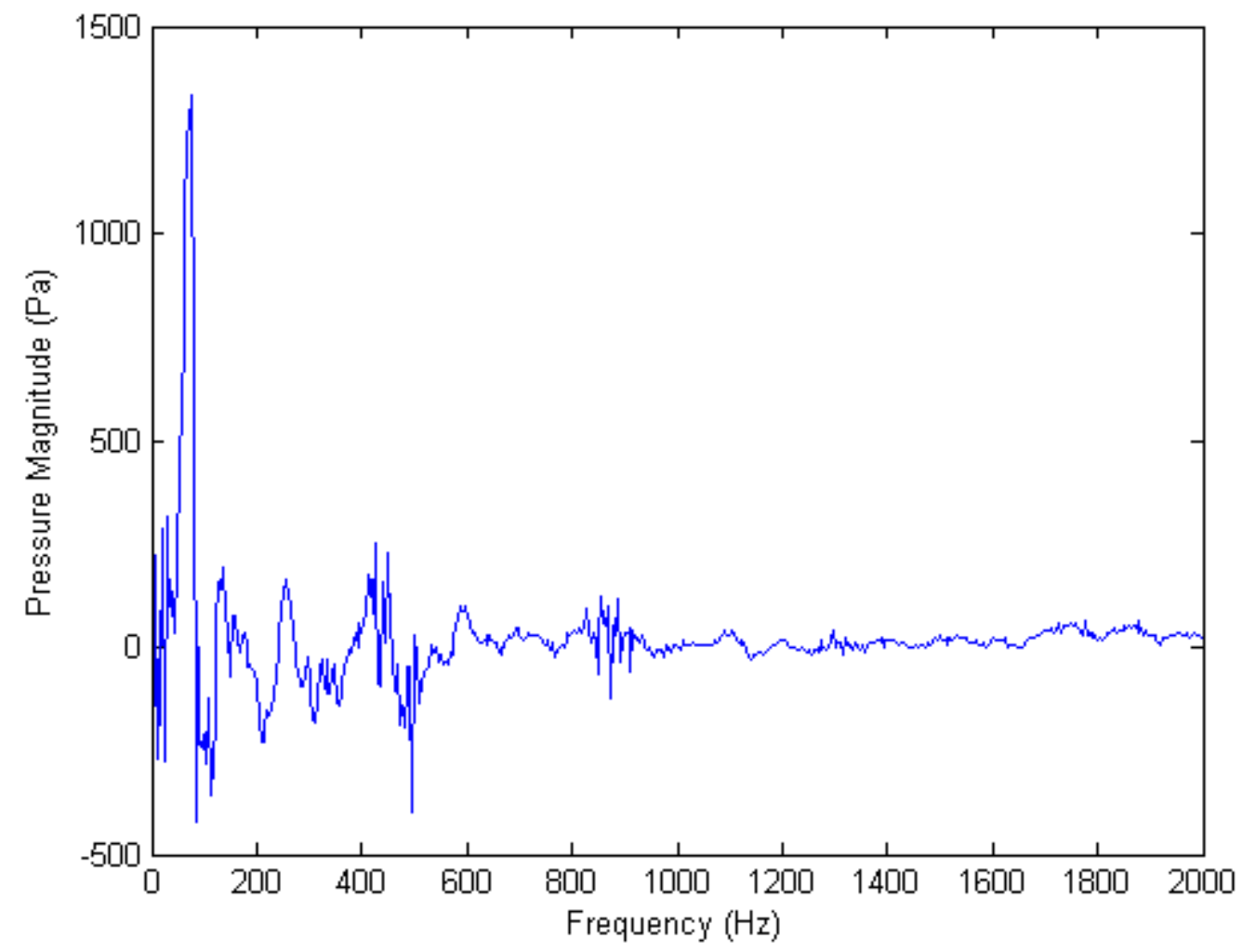

Figure 43: Zoomed in view of the FFT of the pressure readings.

The experimental results contain a much larger fluctuation in sound pressure levels when compared to the BEM results. The actual data is taking in all the sounds including: the sound that gets through the acoustic foam from the room, the hardware used to create the support structure for the plate, and foam oscillating creating pressure waves. 


\section{Driver Head Model}

In an effort to bring this process to the mainstream, a procedure has been setup which will utilize LS-DYNA® and MATLAB ${ }^{\mathrm{TM}}$ to produce an audible sound. The input file used was provided by Dr. Mase and is of a 350cc driver designed in the past. The driver contains 3309 shell elements and can be seen in Figure 44.

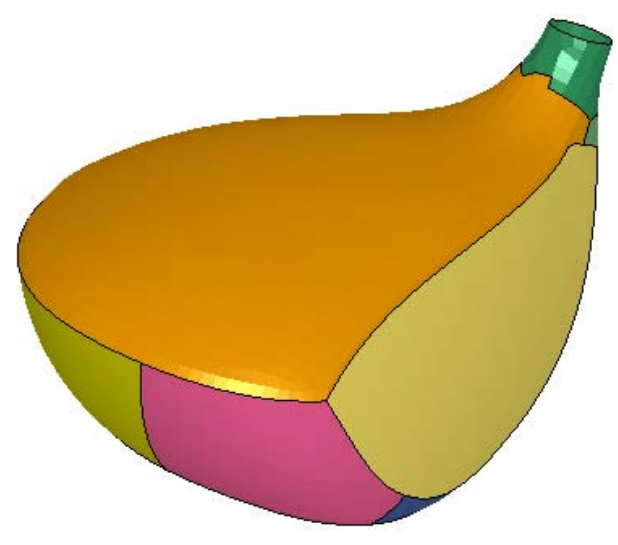

Figure 44: 350ce driver head model.

The driver was paired with the solid golf ball model mentioned earlier. The golf ball was given an initial velocity vectored at the center of the clubface. The file that was run can be seen in Figure 45. 


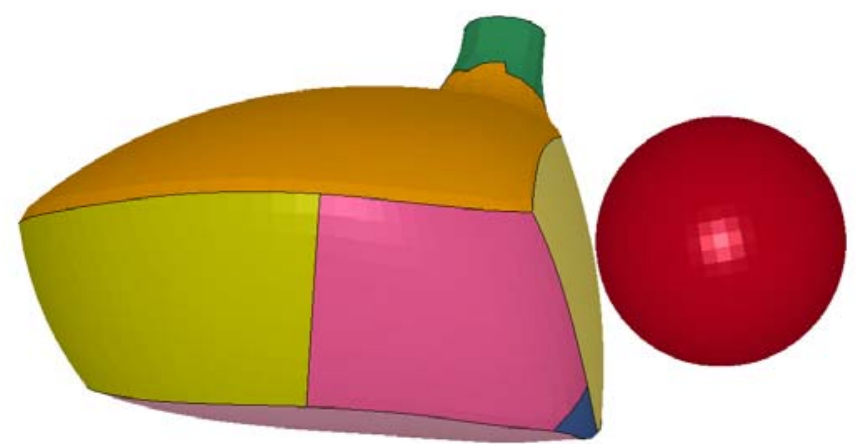

Figure 45: Ball and clubhead impact model.

\section{IV.1 Modal Results}

From the previous validation, modal analysis of the structure can be an indication of the sound produced. The driver head model was fixed at the hosel and LS-DYNA®'s implicit eigenvalue solver gave the modal frequencies and mode shapes. The results are as follows in Figures 46 through 49 .

Freq $=\quad 318.18$

Contours of Resultant Displacement

min $=0$, at node 200094

$\max =0.0394953$, at node 400037

Fringe Levels

$3.950 \mathrm{e}-02$

$3.555 \mathrm{e}-02$

$3.160 e-02$

$2.765 e-02$

$2.370 \mathrm{e}-02$

$1.975 \mathrm{e}-02$

$1.580 e-02$

$1.185 e-02$

$7.899 \mathrm{e}-03$

$3.950 \mathrm{e}-03$

$0.000 \mathrm{e}+00$

Z

W

Figure 46: First mode oscillates around the shaft axis. 
Freq $=\quad 361.07$

Contours of Resultant Displacement

$\min =0$, at node \# 200094

$\max =0.0813703$, at node 400188

Fringe Levels

$8.137 \mathrm{e}-02$

$7.323 \mathrm{e}-02$

$6.510 \mathrm{e}-02$

$5.696 \mathrm{e}-02$

$4.882 \mathrm{e}-02$

$4.069 \mathrm{e}-02$

$3.255 e-02$

$2.441 \mathrm{e}-02$

$1.627 \mathrm{e}-02$

$8.137 \mathrm{e}-03$

$0.000 \mathrm{e}+00$

$z$

Y.X

Figure 47: The second mode oscillates towards "up" getting closer to the golfer's ear and "down" towards the ground.

Contours of Resultant Displacement

$\min =0$, at node\# 200094

$\max =0.040172$, at node $\# 100400$

$4.017 \mathrm{e}-02$

$3.615 \mathrm{e}-02$

$3.214 \mathrm{e}-02$

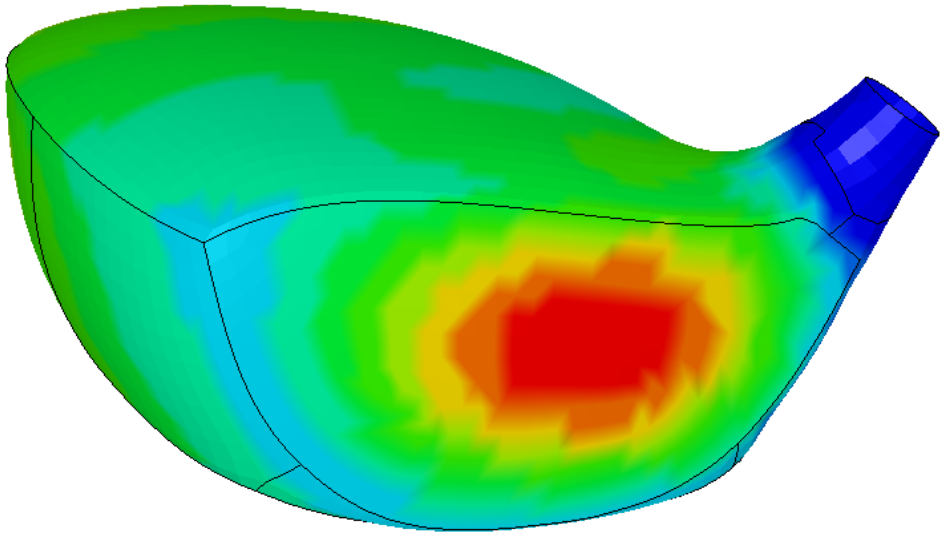

$2.812 \mathrm{e}-02$

$2.410 \mathrm{e}-02$

$2.009 \mathrm{e}-02$

$1.607 \mathrm{e}-02$

$1.205 \mathrm{e}-02$

$8.034 \mathrm{e}-03$

$4.017 \mathrm{e}-03$

$0.000 \mathrm{e}+00$

$z$

W $x$

Figure 48: A frequency of $2508 \mathrm{~Hz}$ excites the face of the driver head. 


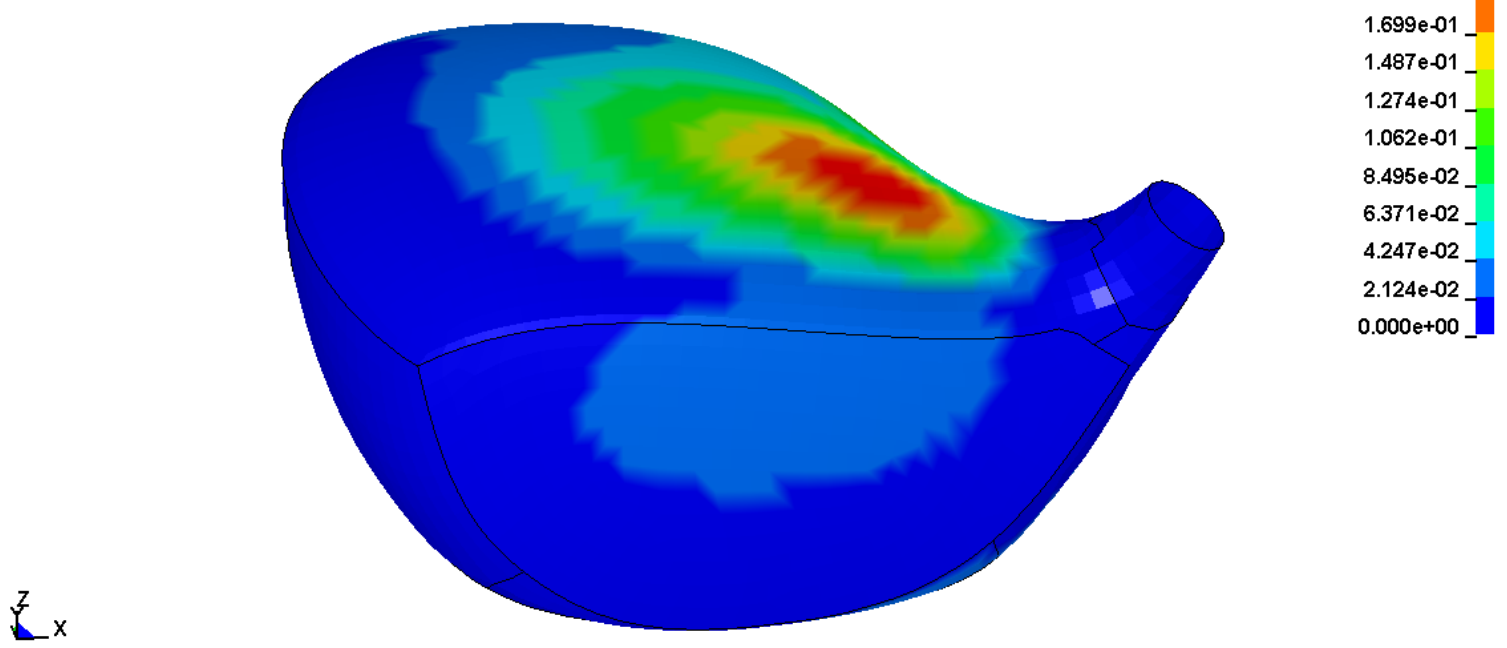

Figure 49: This mode excites the crown of the driver.

The mode shapes produced are similar to those created by Hocknell in his paper [Hocknell]. The placement and frequencies coincide with those previously determined. This is another way to validate the mesh used in the driver simulation.

\section{IV.2 BEM Results}

The acoustic simulation, with the hosel fixed and the reference acoustic point directly behind the driver head, was run on a lab workstation. The simulation was run for a quarter second, and the BEM solution iterated for every fourth frequency from $20 \mathrm{~Hz}$ to $20,000 \mathrm{~Hz}$. The results took about one hundred hours to complete and the resulting sound pressure level is shown in Figure 50. 


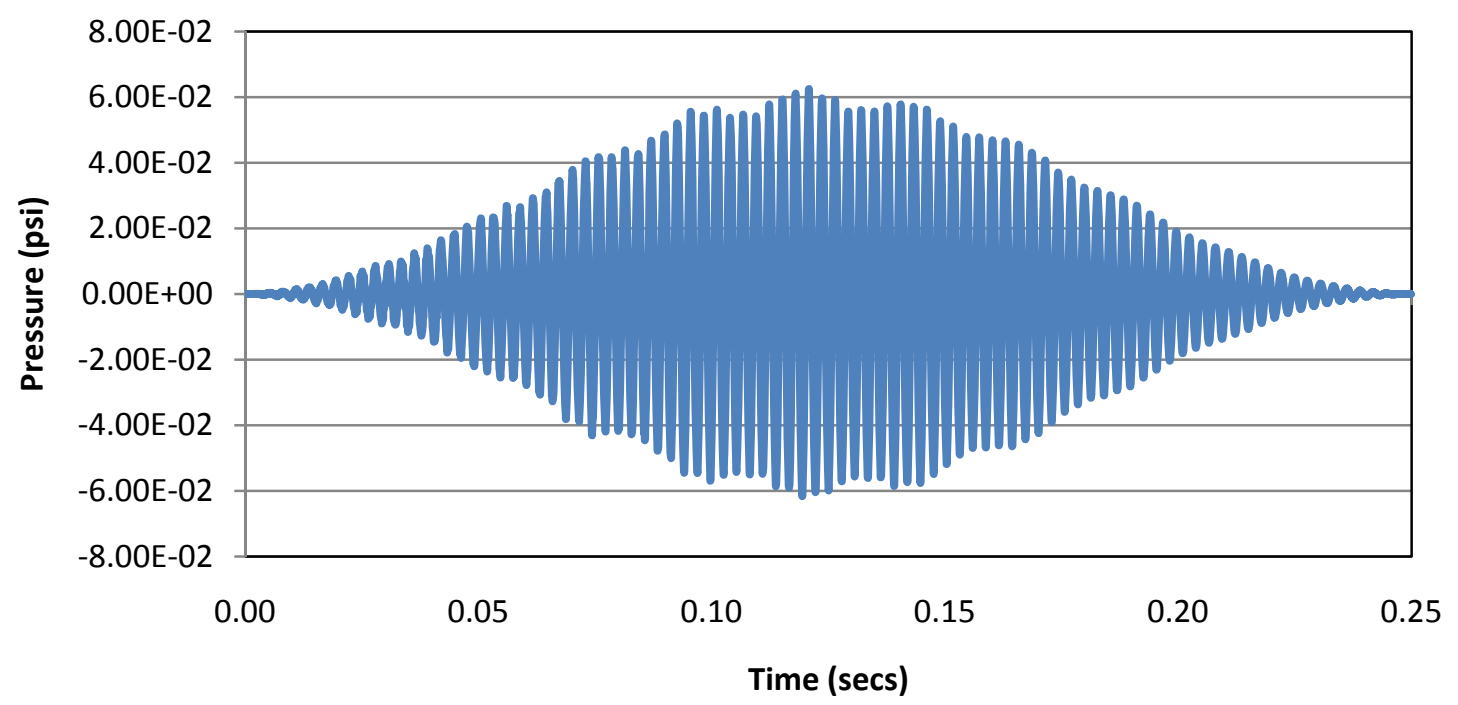

Figure 50: Sound pressure of BEM result.

The sound pressure level from the BEM doesn't intuitively match the expected sound from an impact. The symmetry of the solution comes from the inverse FFT that LS-DYNA® performs get the solution into the temporal domain. The FFT of the signal is shown in Figure 51 and has a single distinct frequency. The frequency from the FFT happens at $356 \mathrm{~Hz}$, which is very close to one of the vibrational modes found during the modal analysis. 


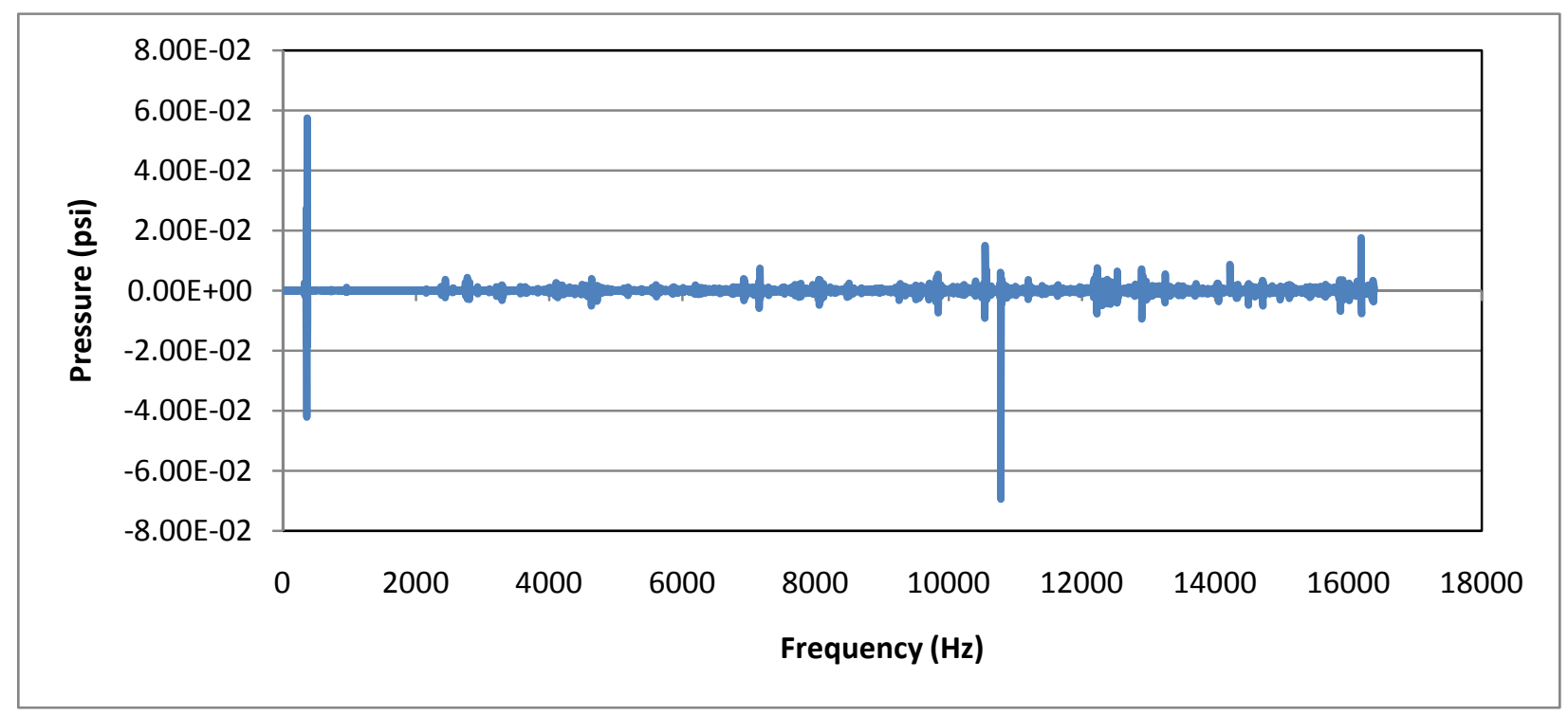

Figure 51: FFT from BEM solution.

\section{IV.3 Experimental Results}

Testing the actual product can give the most accurate results. The following are samples of driver head impacts provided by Nate Radcliff at Cleveland golf. 


\section{IV.3.1 Cobra LD Driver}

The unique features of the Cobra Speed LD driver are its large and thin face paired with center of gravity lowering dimples on the crown. The driver can be seen in Figure 52.

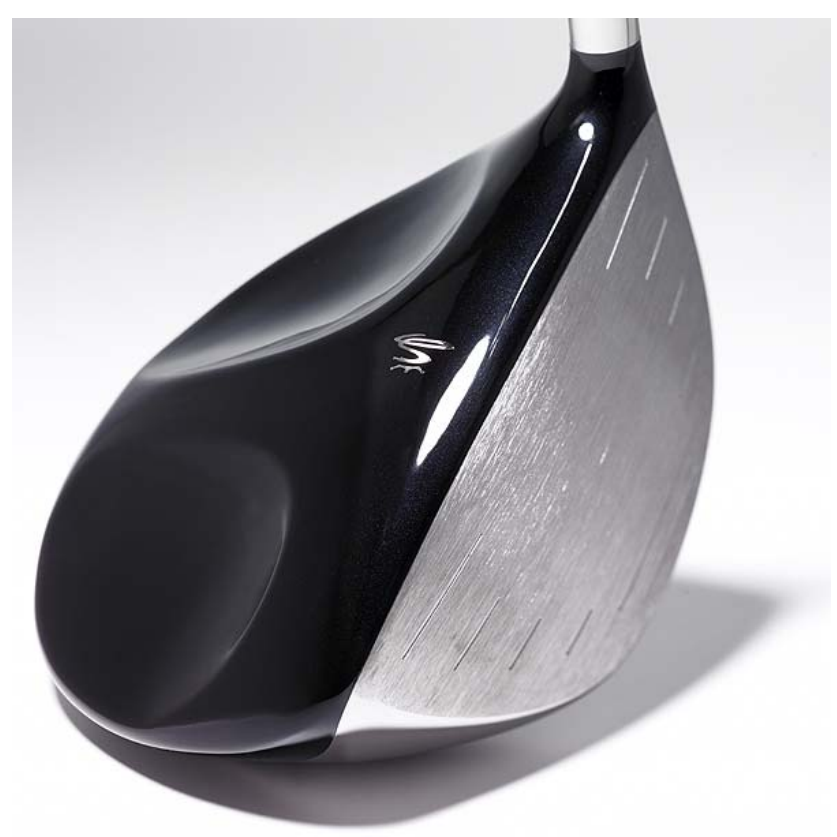

Figure 52: Cobra Speed LD driver from cobragolf.com.

The large and thin face will lower the natural frequency of the face and the dimples on the crown can stiffen the structure. The resulting FFT plot of a golf ball impact can be seen in Figure 53. 


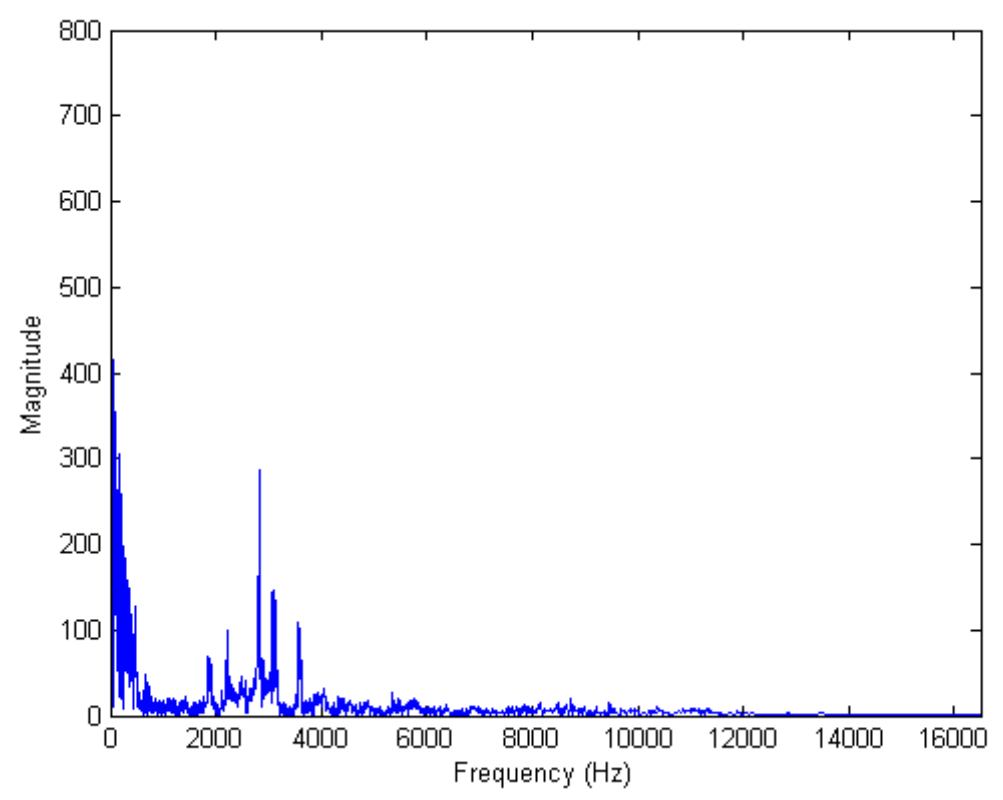

Figure 53: FFT plot of impact of Cobra Speed LD driver.

There is a good amount of noise apparent in the data take by Nate. The result of the FFT from a MATLAB $^{\mathrm{TM}}$ analysis shows distinct low frequency noise along with numerous peaks from the $2000 \mathrm{~Hz}$ to $4000 \mathrm{~Hz}$ range. The reviews of this driver point to the sound not being the most pleasant to the ears. 


\section{IV.3.2 Ping G5 Driver}

The Ping G5 has a more traditional shape and design than the Cobra. The crown of the driver is smooth and has a hemispherical shape although the crescent moon graphic in Figure 54 makes it look otherwise.

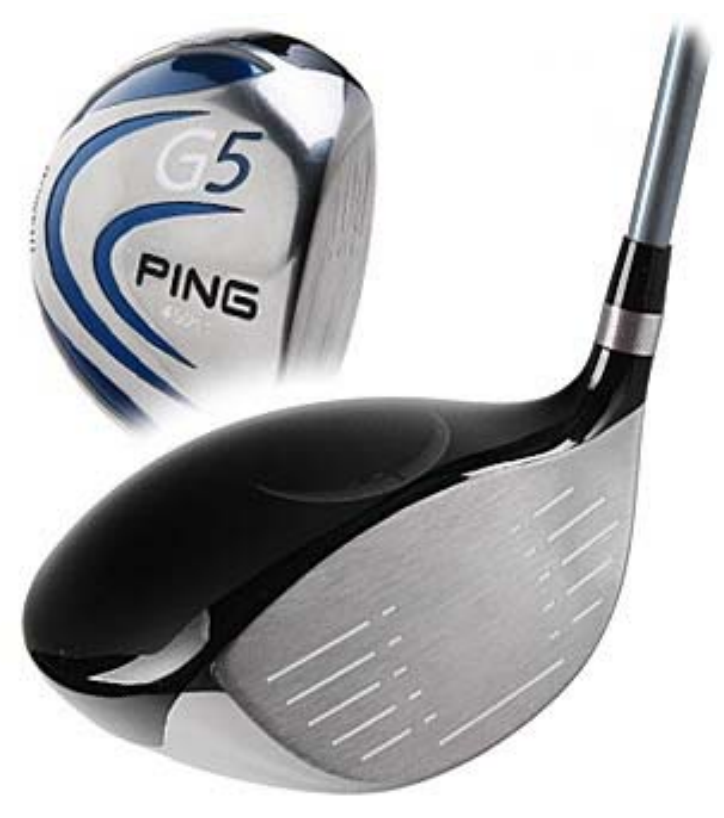

Figure 54: Ping G5 driver from pinggolf.com.

Most of the key features are hidden inside the head with its internal weighting and variable face thickness. The result FFT of the sound can be found in Figure 55. 


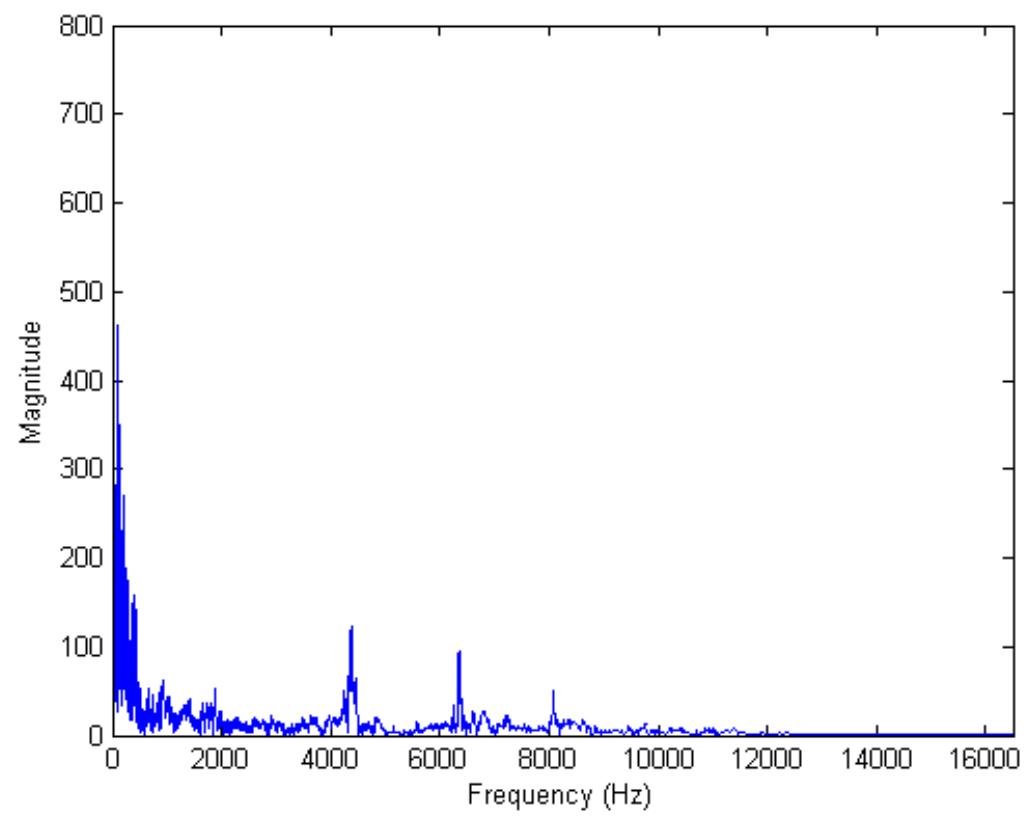

Figure 55: FFT of Ping G5 impact.

The FFT of the Ping G5 impact is much cleaner than the Cobra LD. There is quite a bit on the low end of the spectrum. This may be attributed to the sound created by the ball at impact. The distinct frequencies are $4375 \mathrm{~Hz}, 6350 \mathrm{~Hz}$, and $8080 \mathrm{~Hz}$. While this does not characterize the club as a "good" or "bad", knowing the frequencies produced can aide in further designs. 


\section{IV.3.3 Cleveland Launcher DST Driver}

The Cleveland driver is a more traditional shape shown in Figure 56. The resulting FFT plot of impact is found in Figure 57. The Cleveland Launcher has two distinct frequencies that occur at $4090 \mathrm{~Hz}$ and $6070 \mathrm{~Hz}$.

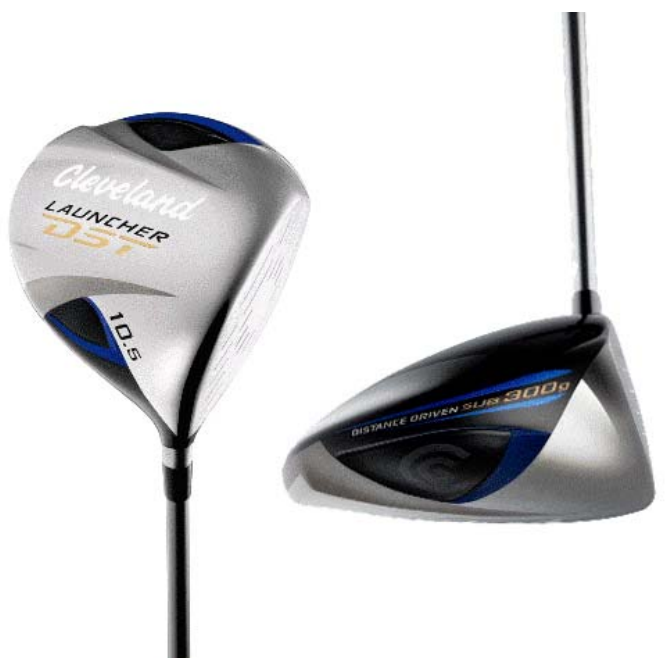

Figure 56: Cleveland Launcher DST driver from clevelandgolf.com.

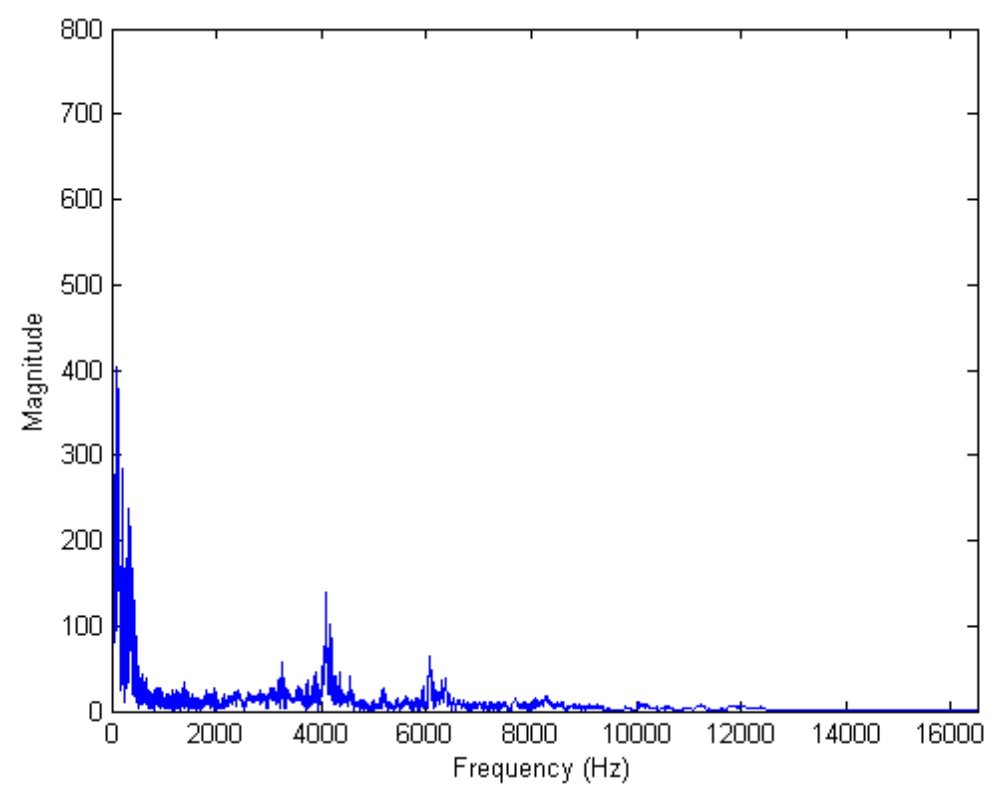

Figure 57: FFT of Cleveland Launcher DST impact. 


\section{Conclusions}

When comparing the data taken during the air cannon test, the acoustic model did not match up with experimental data. Looking at high speed film of the impact hitting the support structure, one could see most of the deformation comes from the two crossbars supporting the plate. From that, the two crossbars were incorporated into the acoustic simulation. The acoustic simulation was run again and still found to not match the data collected. The final resolution was to model the entire structure. This was not an ideal experimental setup, since the validation turned from determining the sound of a titanium plate impacted by a golf ball to the sound a titanium plate and its entire support structure makes when impacted with a golf ball.

To solve the problem, the support structure should be an order of magnitude stiffer than the part it is supporting. The only solution is larger, thicker pieces of metal. Of course the addition of material and time would add to the costs of the overall project. The lesson is to not always skimp on a design. Having done it the right way would have simplified our model and greatly decreased computational time.

With that being said, the BEM did produce an FFT plot that contained frequencies that were measured during the experiment. When compared to the acoustic data, the plot was not fully populated with frequencies since the model didn't account for the golf ball or sound that was getting through the acoustic foam. On a good note, the spikes in the simulated FFT plot did coincide with spikes from the actual measured sound. This did somewhat validate that the BEM solver in LS-DYNA® can predict some of the sound from an impact simulation.

The exact sound of a golf ball at impact is difficult to simulate. All the components of the club will factor into the unique sound of each driver. This thesis looked at the driver head alone and 
the sound produced from a simple impact simulation. The sound produced from the BEM pressure plot when run in MATLAB ${ }^{\mathrm{TM}}$ does not sound like a golf ball impact. The resulting sound is very "clean" when heard through the computer's speakers. If we had introduced the golf ball and the walls in the room to the BEM solver the resulting sound would have been better, but our computational time would drastically increase.

What it does do, is highlight the exact frequency the driver head makes in that specific loading condition (i.e. impact in the center and off-center positions). Quantifying a driver impact in the real world and comparing it to the BEM result can show a relation between a "good" and "bad" sound. Once the "good" sound of a driver is determined from the BEM, the model can be optimized towards that "good" frequency. The designer could then show with confidence the driver head design falls with a desirable specified range. 


\section{References}

"Golf Ball History." Golf Europe: Golf Courses in Ireland, Scotland, England and more. Web. 04 Dec. 2009. <http://www.golfeurope.com/almanac/history/golf_ball.htm>.

"TaylorMade Golf - Golf History." TaylorMade Golf - Clubhouse. http://www.taylormadegolf.com/mainlevel/golfshop.html\#501>.

Alia, Ahlem, and Mhamed Souli. "Acoustic and Vibroacoustic Modeling in LS-DYNA® Based on Variational BEM." Proc. of 5th European LS-DYNA® Users Conference.

Axe, J. D., K. Brown, and K. Shannon. "The Vibrational Mode Structure of a Golf ball." Science and Golf IV. 2002. Print.

Biesen, Eric D. "Prediction of Plastic Deformation in Aluminum Softball Bats Using Finite Element Analysis." Thesis. Washington State University, 2006.

Herrin, D. W., F. Matinus, T. W. Wu, and A. F. Seybert. "A New Look at the High Frequency Boundary Element and Rayleigh Integral Approximations." N. pag. Society of Automotive Engineers, 2003.

Hocknell, A., S. R. Mitchell, R. Jones, and S. J. Rothberg. "Hollow Golf Club Head Modal Characteristics: Determination and Impact Applications." Experimental Mechanics 38.2 (1998): 140-46. Print.

Huang, Yun. LSTC. A New Acoustic Solver Based on Boundary Element Method in LS-DYNA®. 
Hunt, John T., Max R. Knittel, Charles S. Nichols, and Don Barach. "Finiete-element approach to acoustic scattering from elastic structures." Naval Undersea Center (1974)

LSTC. LS-DYNA® Keyword User's Manual. Vol. 1. 2007.

LSTC. LS-DYNA® Theory Manual. 2006.

Mase, T., Private conversation through NDA.

Mase, T. and Kersten, A. M, 'Experimental evaluation of a 3-D hyperelastic, rate-dependent golf ball constitutive model," The Engineering of Sport 5 - Conference Proceedings of, Vol. 2, pp. $238-244,2004$

McMaster Carr. Web. <www.mcmaster.com>.

Rainsberger, Robert. TrueGrid® User's Manual: A Guide and a Reference. Vol. 2.

Shannon, K., and J. D. Axe. "On the Acoustic Signature of Golf Ball Impact." Science of Golf IV. 2002. Print.

Sharpe, Roger. "Class notes from ME 404: Finite Element Analysis.” 2008.

Sharpe, Roger. “ME 346 Lab Experiment: Polytropic Blowdown.” 2007.

Smith, D.C., and Bernhard, R.J., 1989, "Verification of Numerical Acoustic Radiation Predictions", Proceedings of the 1989 Noise and Vibration Conference, SAE Conference, $445-452$.

Springer Handbook of Acoustics (Springer Handbook of). New York: Springer, 2007. Print. 
Volkoff-Shoemaker, Nickolai. "Feasibility of Acoustical Optimization with Finite Element Analysis of a Golf Club Driver Head: A Thesis.” 2009

Wilton, D. T. "Acoustic Radiation and Scattering from Elastic Structures." International Journal for Numerical Methods in Engineering 13 (1978): 123-38. 


\section{Appendix A: Drawings}

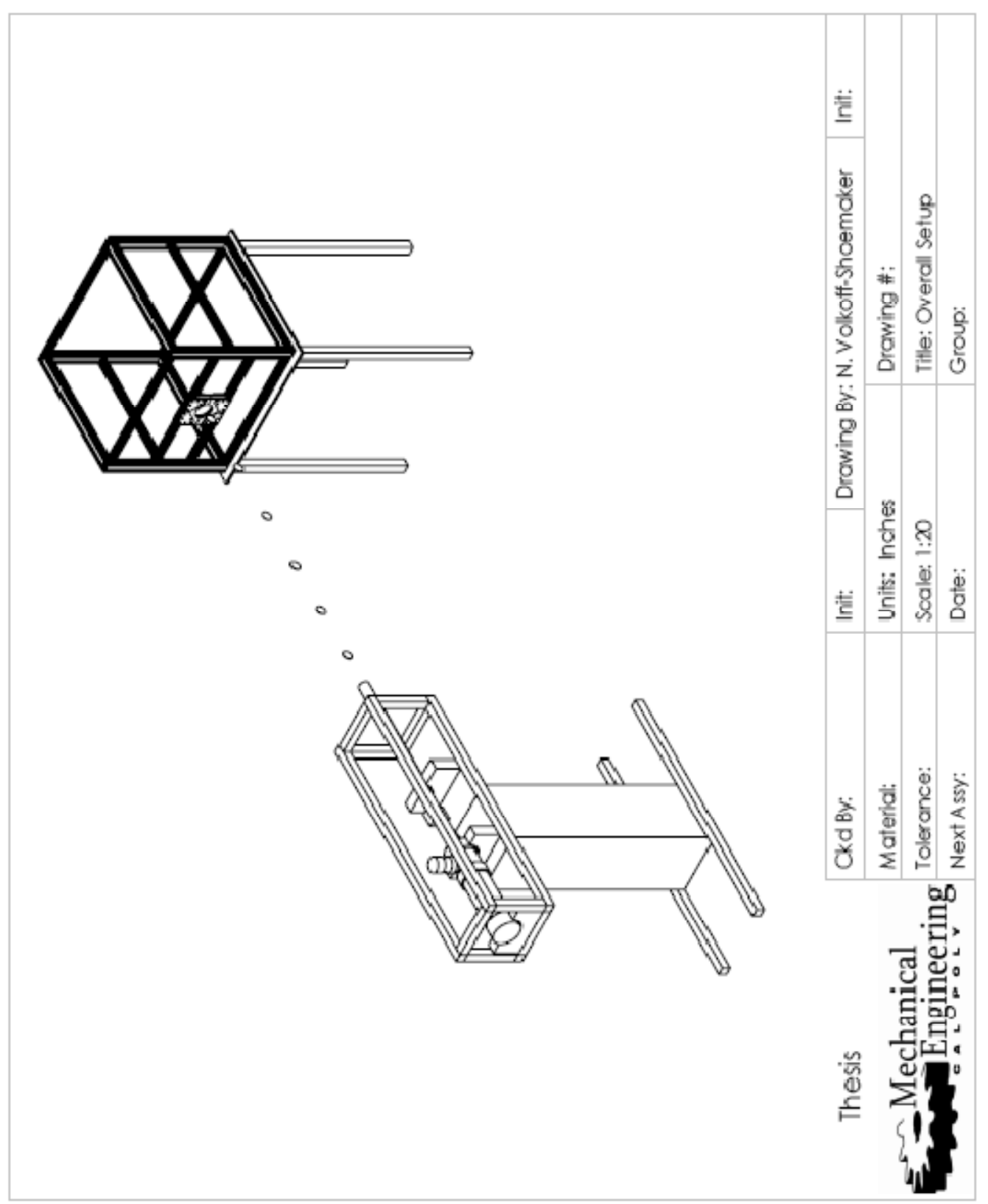



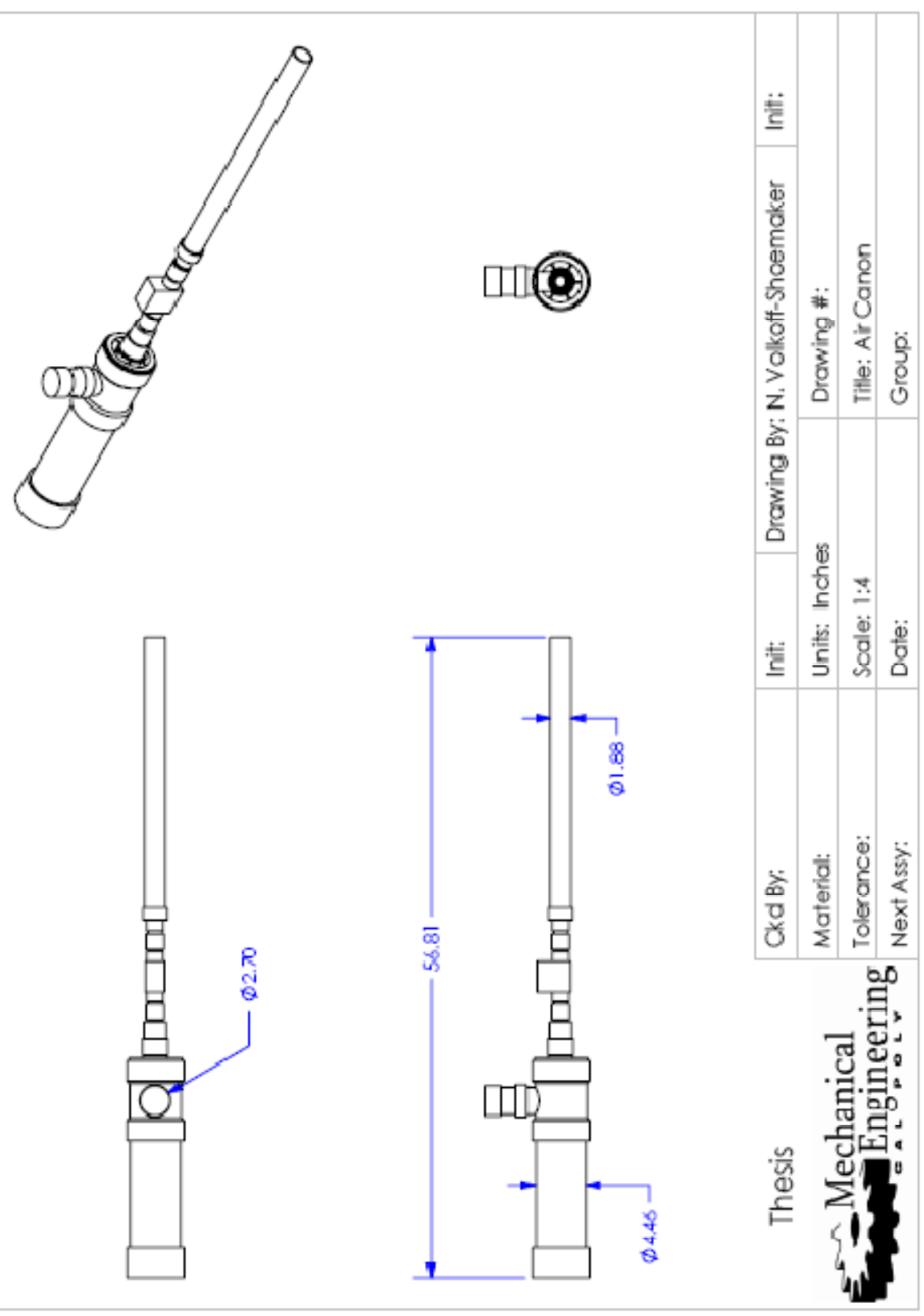


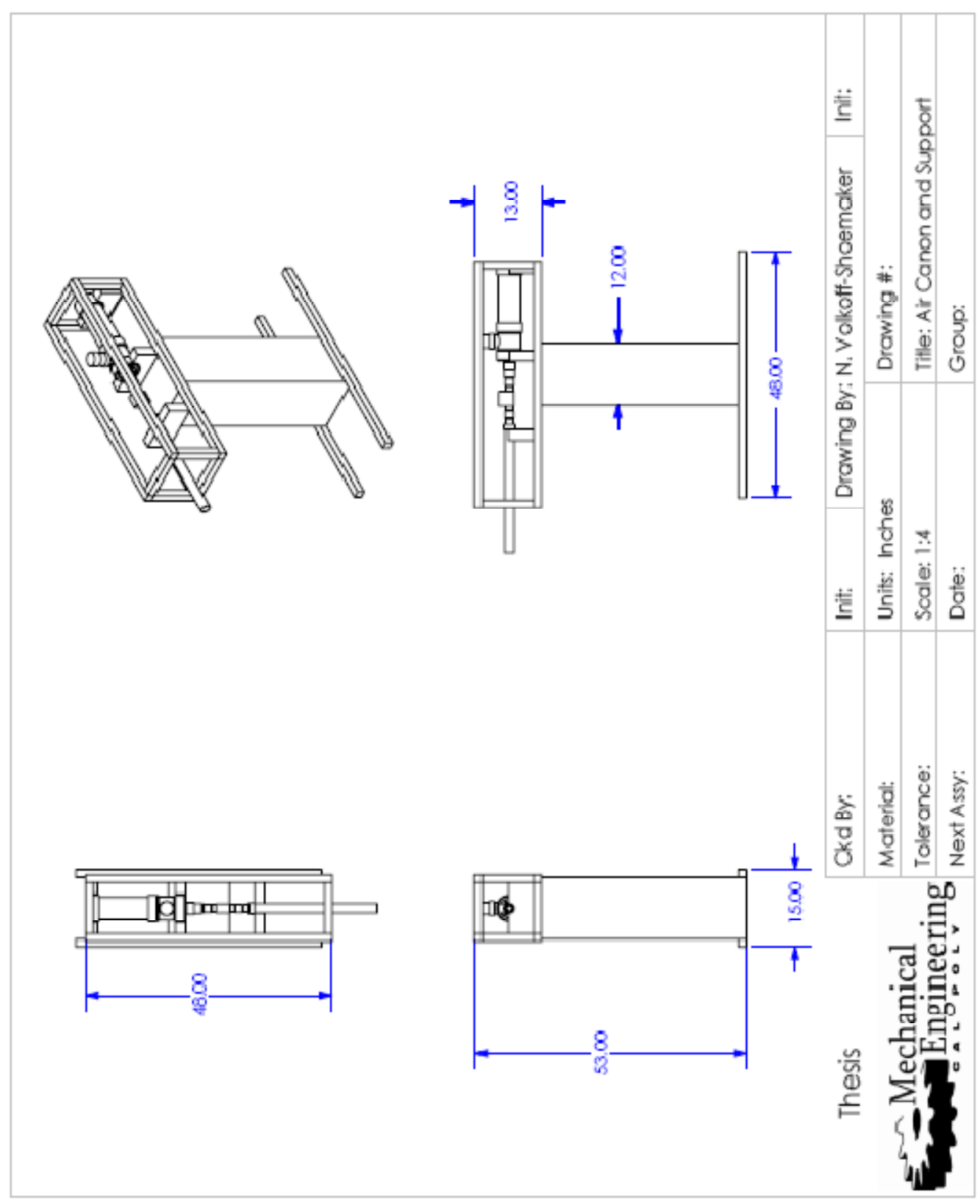




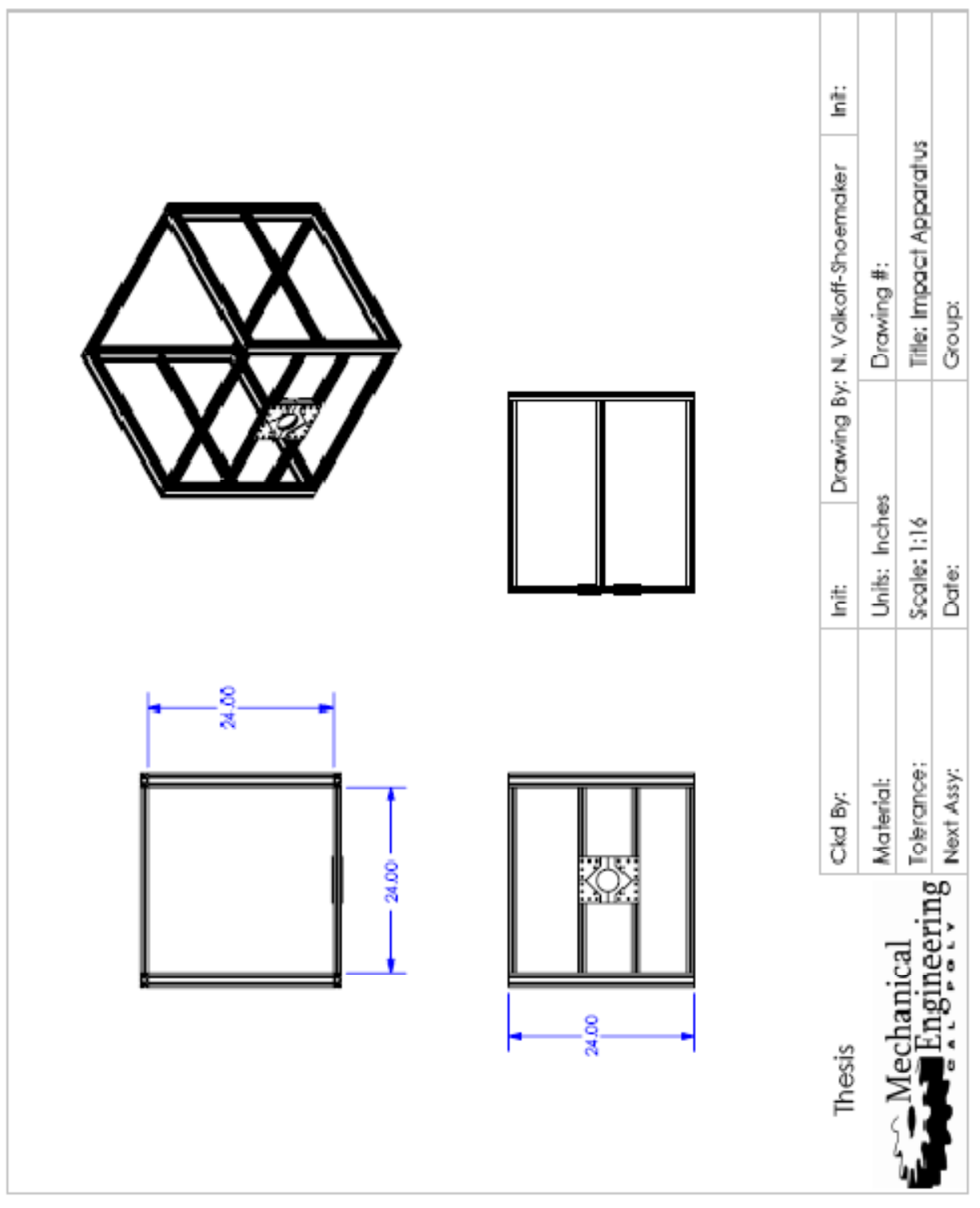




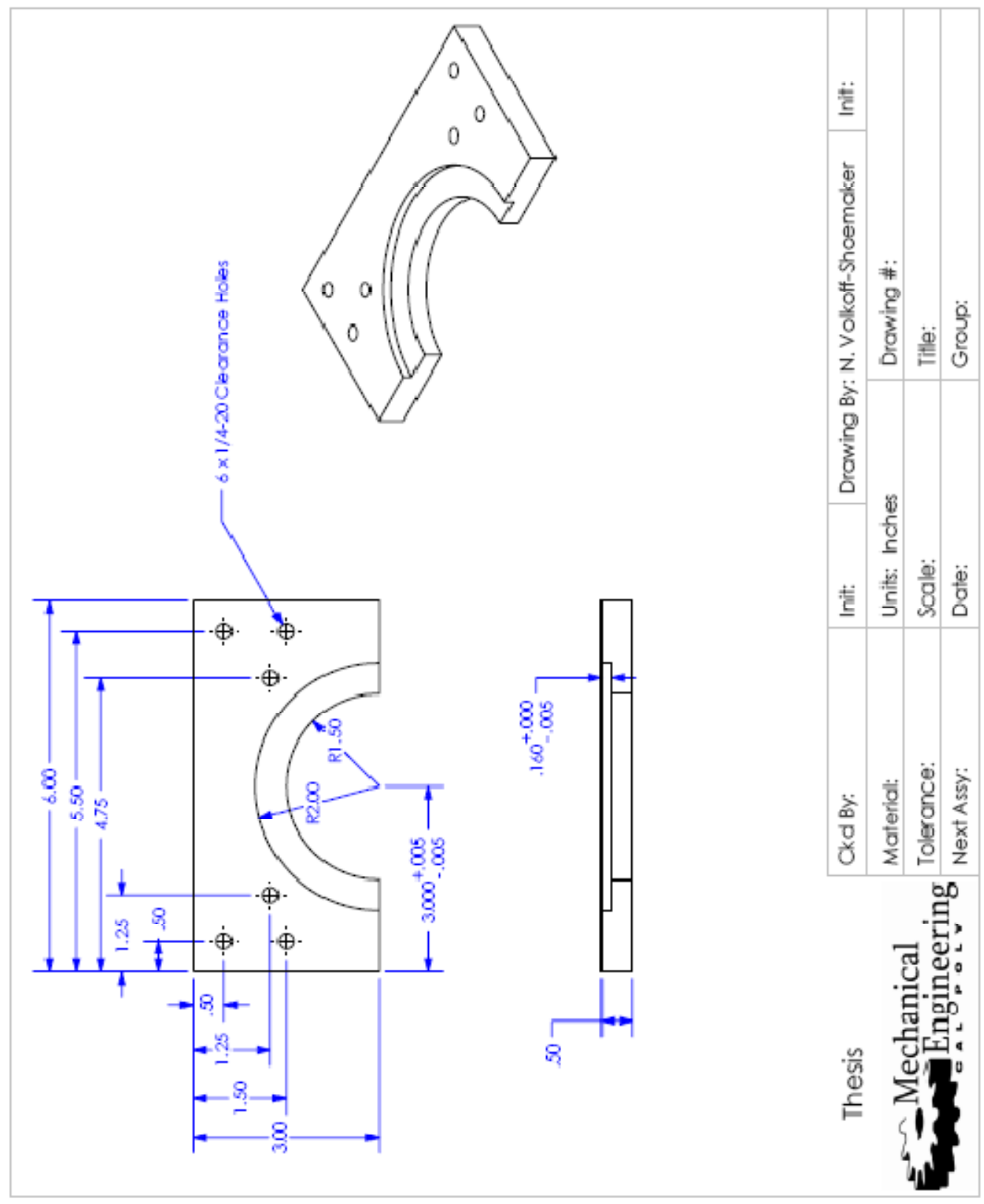




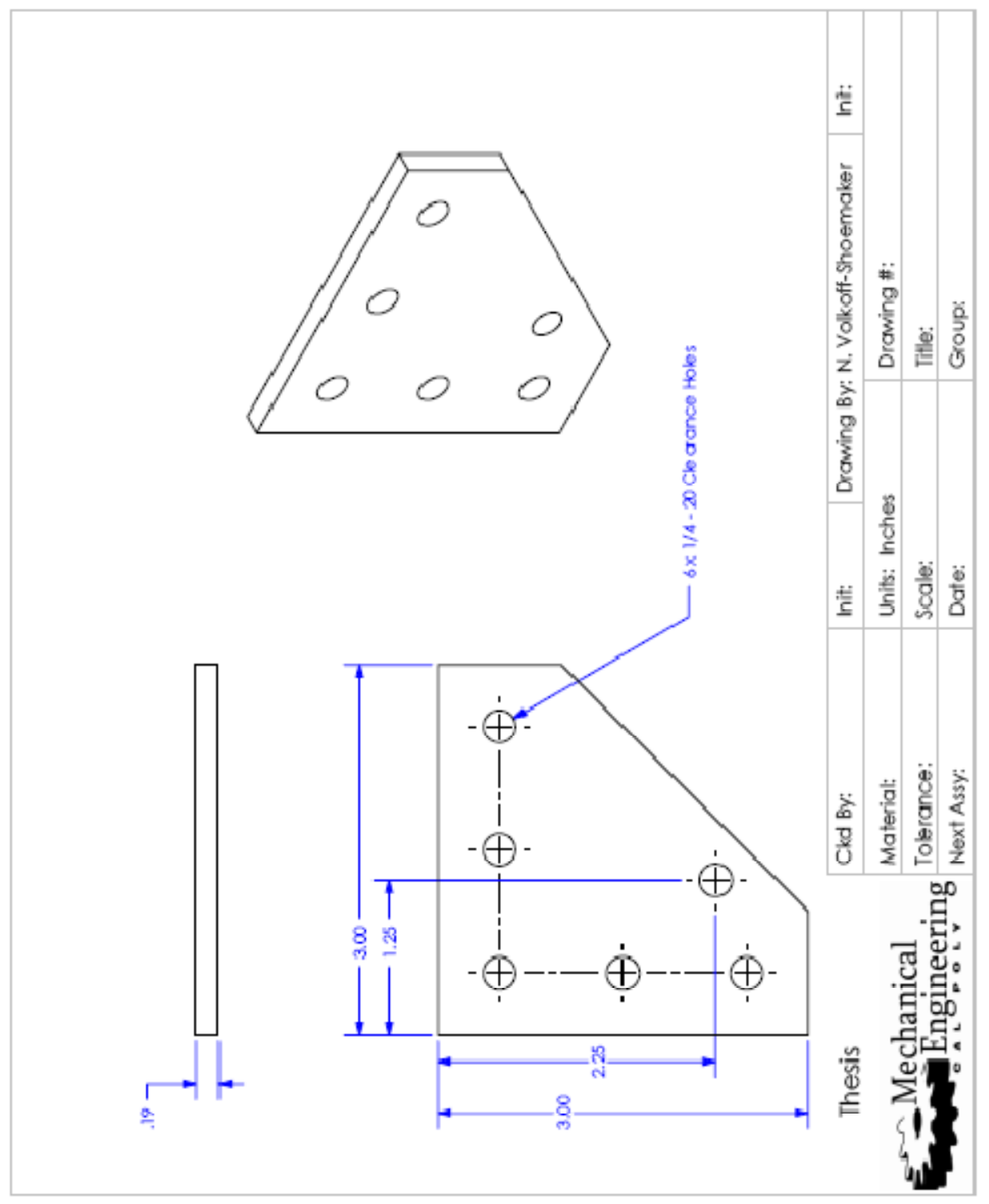



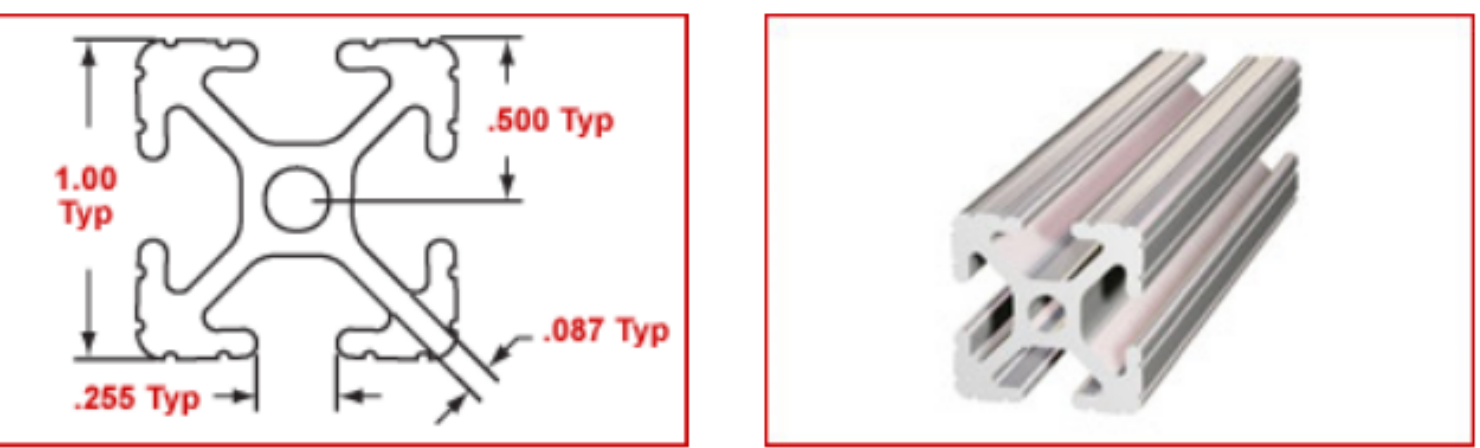

1010 is a $1.0^{\prime \prime} \times 1.0^{\prime \prime}$ T-slotted aluminum profile made from $6105-T 5$ aluminum. This profile has open T-slots on all four sides and is compatible with all 10 Series fasteners and accessories.

\begin{tabular}{|c|c|c|c|c|c|}
\hline Part No. & Finish & Ibs. / Ft. & Stock Length & Homent of Inertia & Area \\
\hline 1010 & Clear Anodized & .5097 & 97,145 or $242 \ln$. & $\begin{array}{l}\mid X=.0442^{\prime \prime \wedge} 4 \\
\mid Y=.0442^{\prime \prime \wedge} 4\end{array}$ & $.4379 \mathrm{Sq} . / \mathrm{ln}$. \\
\hline
\end{tabular}




\section{Appendix B: TrueGrid ${ }^{\circledR}$ Files}

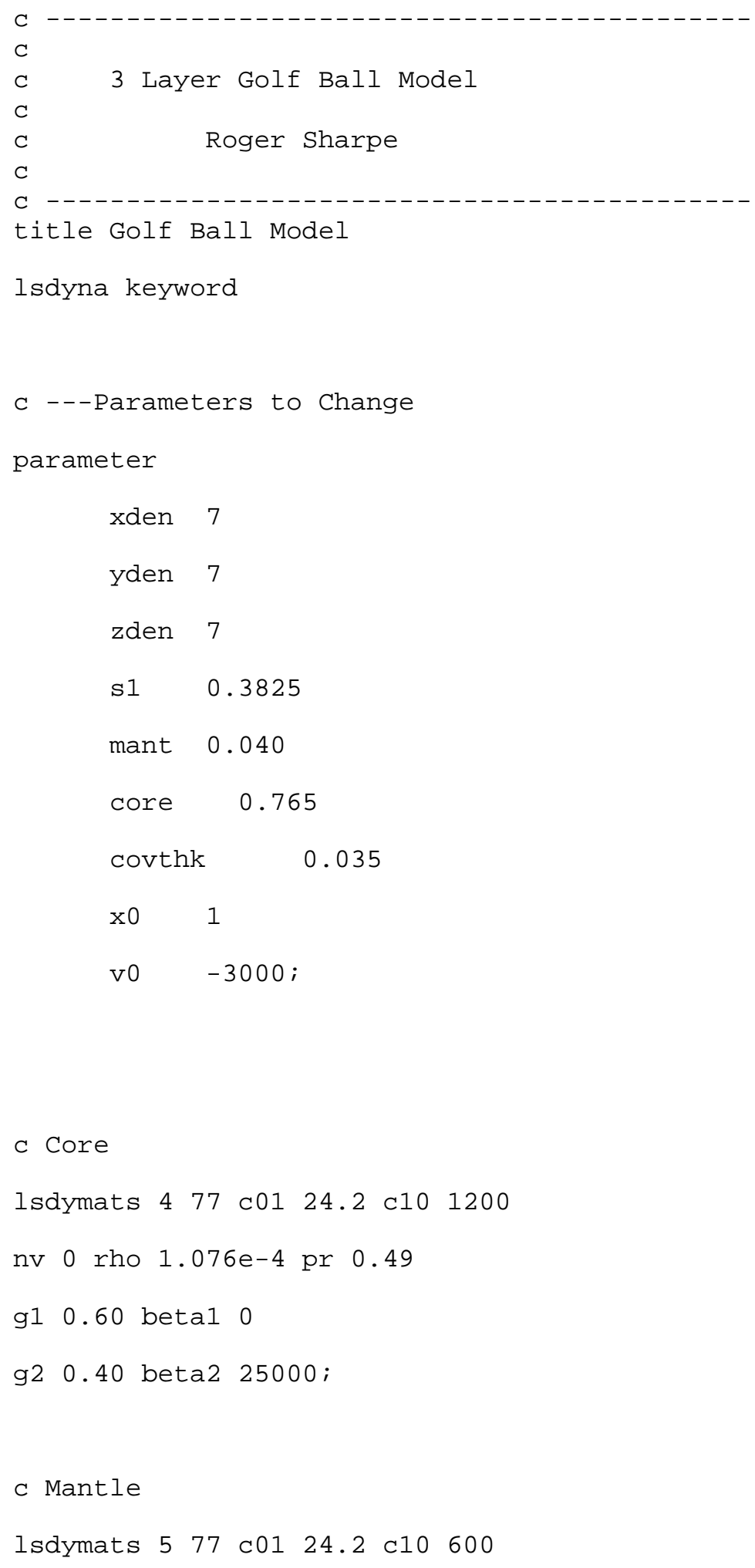




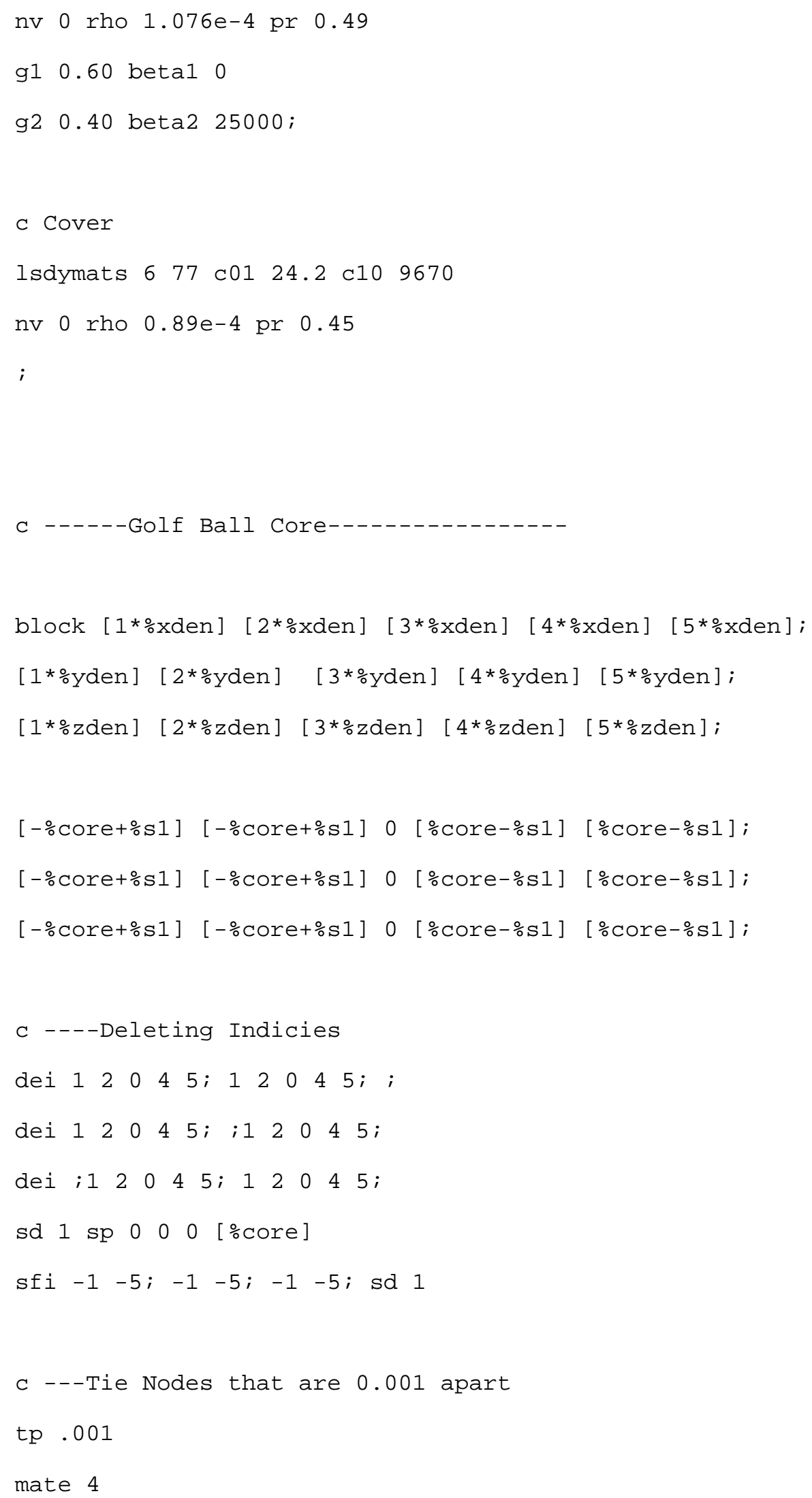




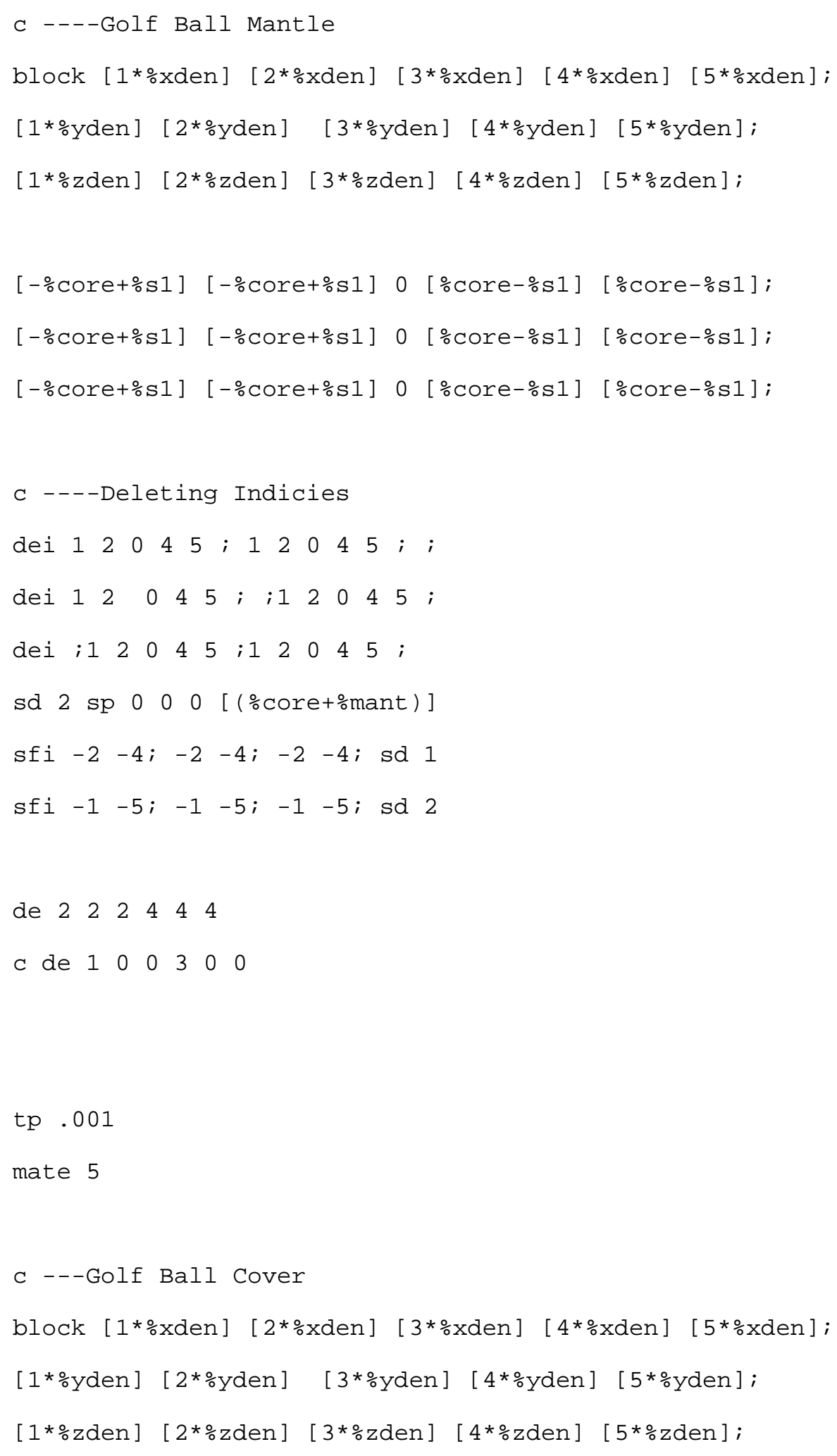




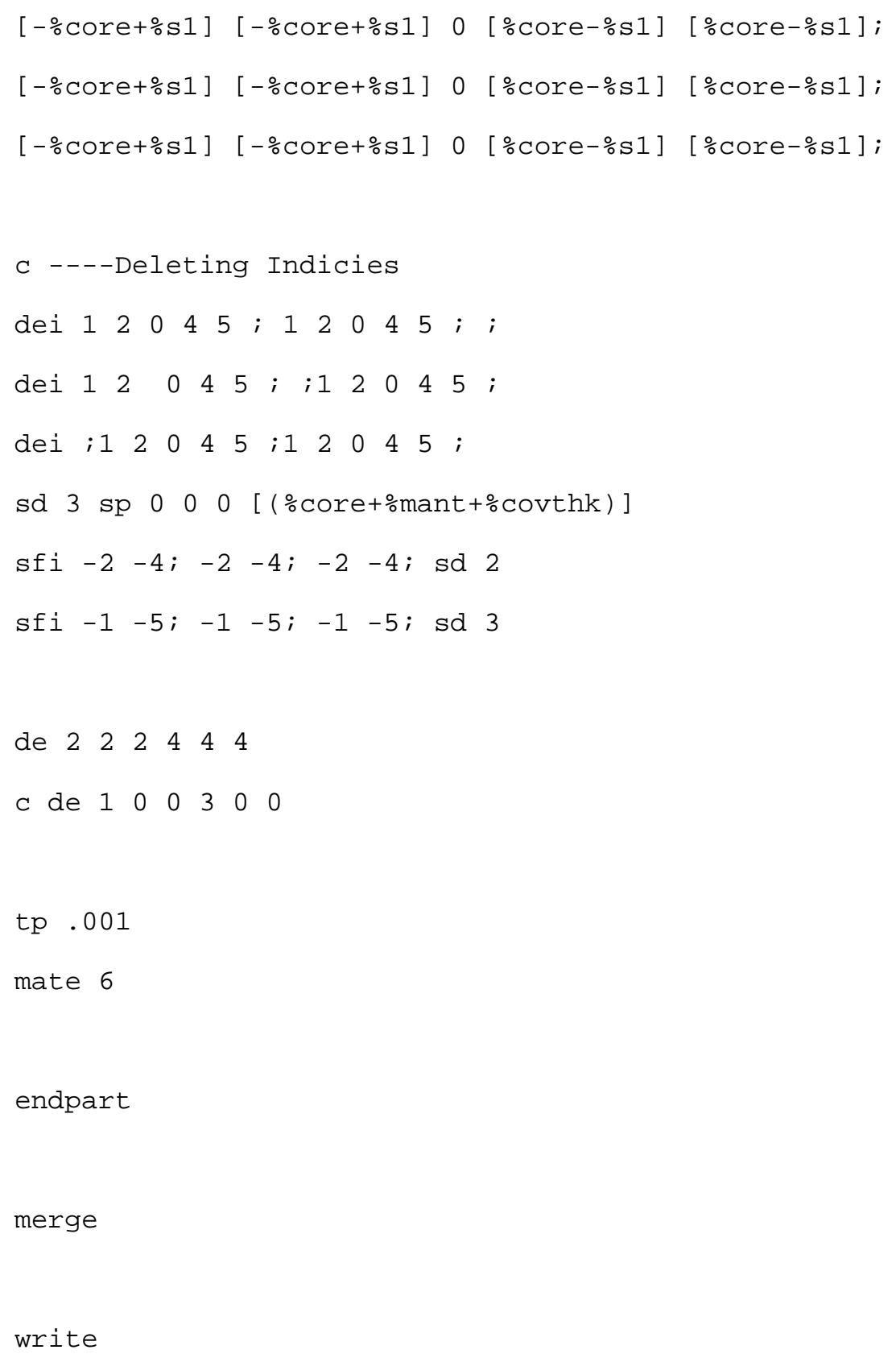




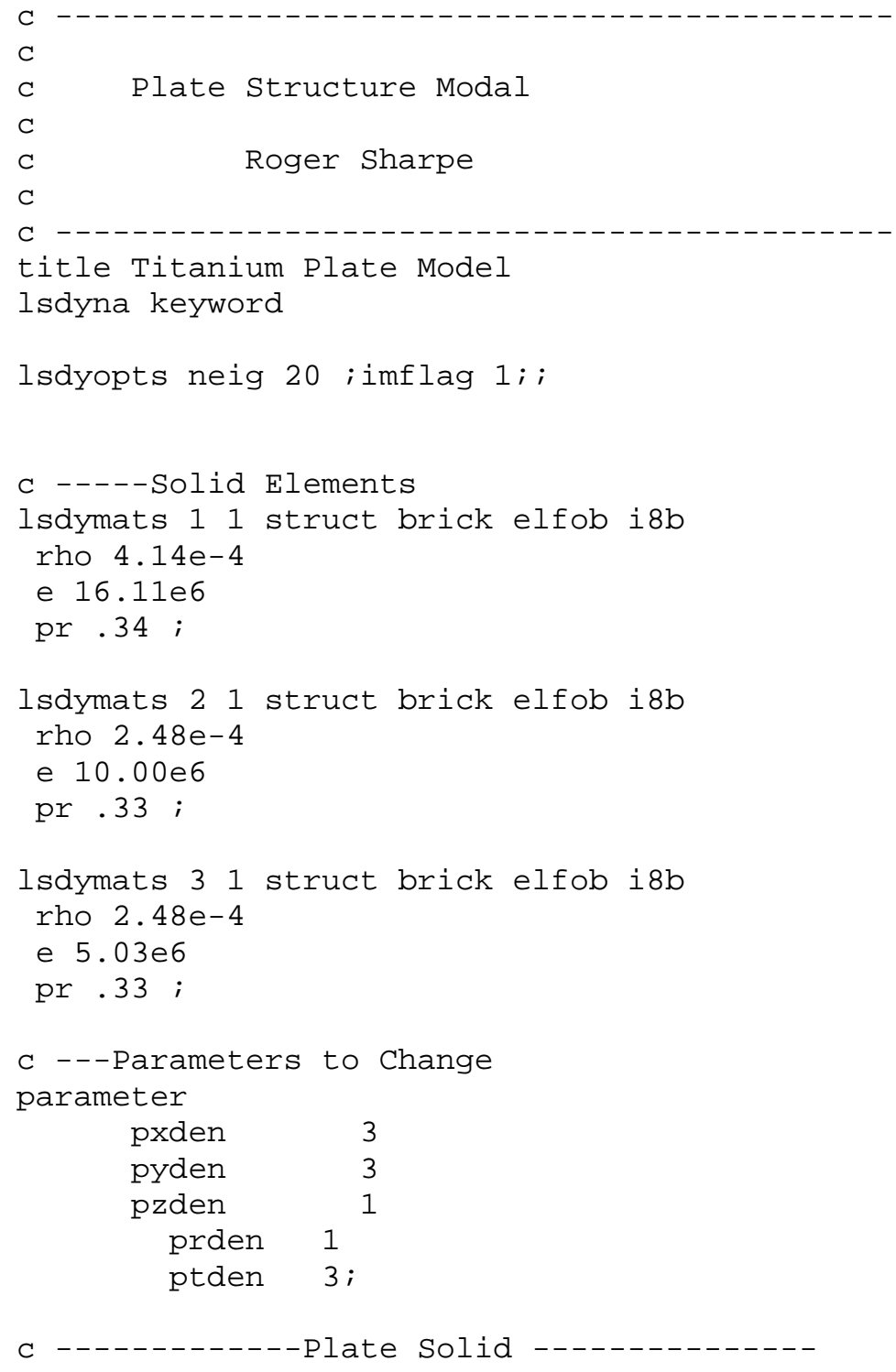




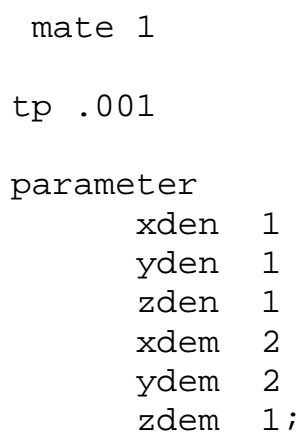

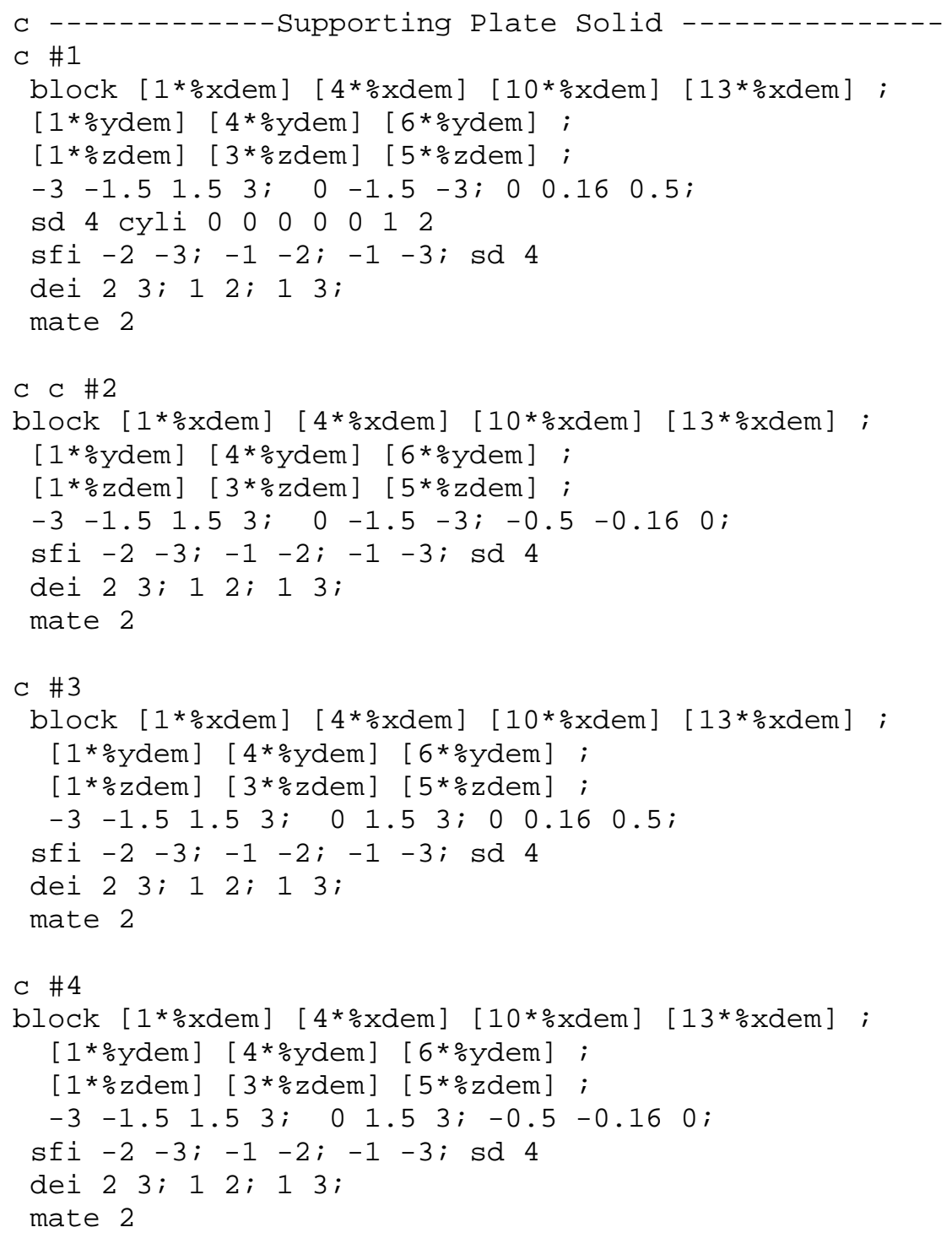

C Rimgs of Alumimum om bottom of $\mathrm{Ti}$ cylinder [1*\%pxden] [2*\%xden]; [1*\%ptden] [5*\%ptden] [9*\%ptden] [13*\%ptden] [17*\%ptden] ; [1*\%pzden] [3*\%pzden] ; 
mate 2

c Rings of Aluminum on top of $\mathrm{Ti}$

cylinder [1*\%pxden] [2*\%pxden];

[1*\%ptden] [5*\%ptden] [9*\%ptden] [13*\%ptden] [17*\%ptden] ;

[1*\%pzden] [3*\%pzden];

$1.52 ; 090180270360 ; 0.160 .5 ;$

mate 2

mate 2

c Support arms

block [1*\%xden] [10*\%xden] [16*\%xden] [28*\%xden] [34*\%xden] [44*\%xden];

[1*\%yden] [4*\%yden];

[1*\%zden] [3*\%zden] [5*\%zden] [7*\%zden] [9*\%zden];

$\begin{array}{lllllll}-12 & -3 & -1.5 & 1.5 & 3 & 12 ; & 3\end{array}$; $-0.5-0.1600 .160 .5 ;$

mate 3

block [1*\%xden] [10*\%xden] [16*\%xden] [28*\%xden] [34*\%xden] [44*\%xden];

[1*\%yden] [4*\%yden];

[1*\%zden] [3*\%zden] [5*\%zden] [7*\%zden] [9*\%zden];

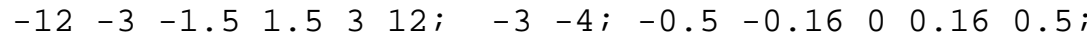

mate 3

C Rest of Structure

c Left Front Vertical Support

block [1*\%xden] [8*\%xden];

[1*\%yden] [4*\%yden] [11*\%yden] [14*\%yden] [17*\%yden] [20*\%yden] [23*\%yden] [26*\%yden] [33*\%yden] [36*\%yden];

[1*\%zden] [3*\%zden] [5*\%zden] [7*\%zden] [9*\%zden];

$\begin{array}{llllllllllllllllll}-14 & -12 ; & -12 & -11 & -4 & -3 & -0.5 & 0.5 & 3 & 4 & 11 & 12 ; & -0.5 & -0.16 & 0 & 0.16 & 0.5 ;\end{array}$

mate 3

c Right Front Vertical Support

block [1*\%xden] [8*\%xden];

[1*\%yden] [4*\%yden] [11*\%yden] [14*\%yden] [17*\%yden] [20*\%yden] [23*\%yden]

[26*\%yden] [33*\%yden] [36*\%yden];

[1*\%zden] [3*\%zden] [5*\%zden] [7*\%zden] [9*\%zden];

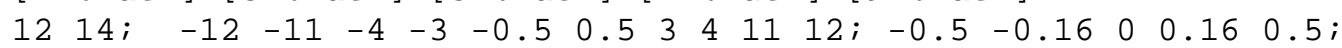

mate 3

c Front Top Horizontal Support

block [1*\%xden] [10*\%xden] [13*\%xden] [19*\%xden] [22*\%xden] [32*\%xden];

[1*\%yden] [4*\%yden];

[1*\%zden] [3*\%zden] [5*\%zden] [7*\%zden] [9*\%zden];

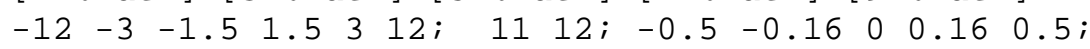

mate 3

c Front Bottom Horizontal Support

block [1*\%xden] [10*\%xden] [13*\%xden] [19*\%xden] [22*\%xden] [32*\%xden];

[1*\%yden] [4*\%yden];

[1*\%zden] [3*\%zden] [5*\%zden] [7*\%zden] [9*\%zden];

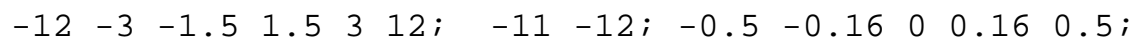

mate 3

C Top Left Side Support

block [1*\%xden] [8*\%xden]; 


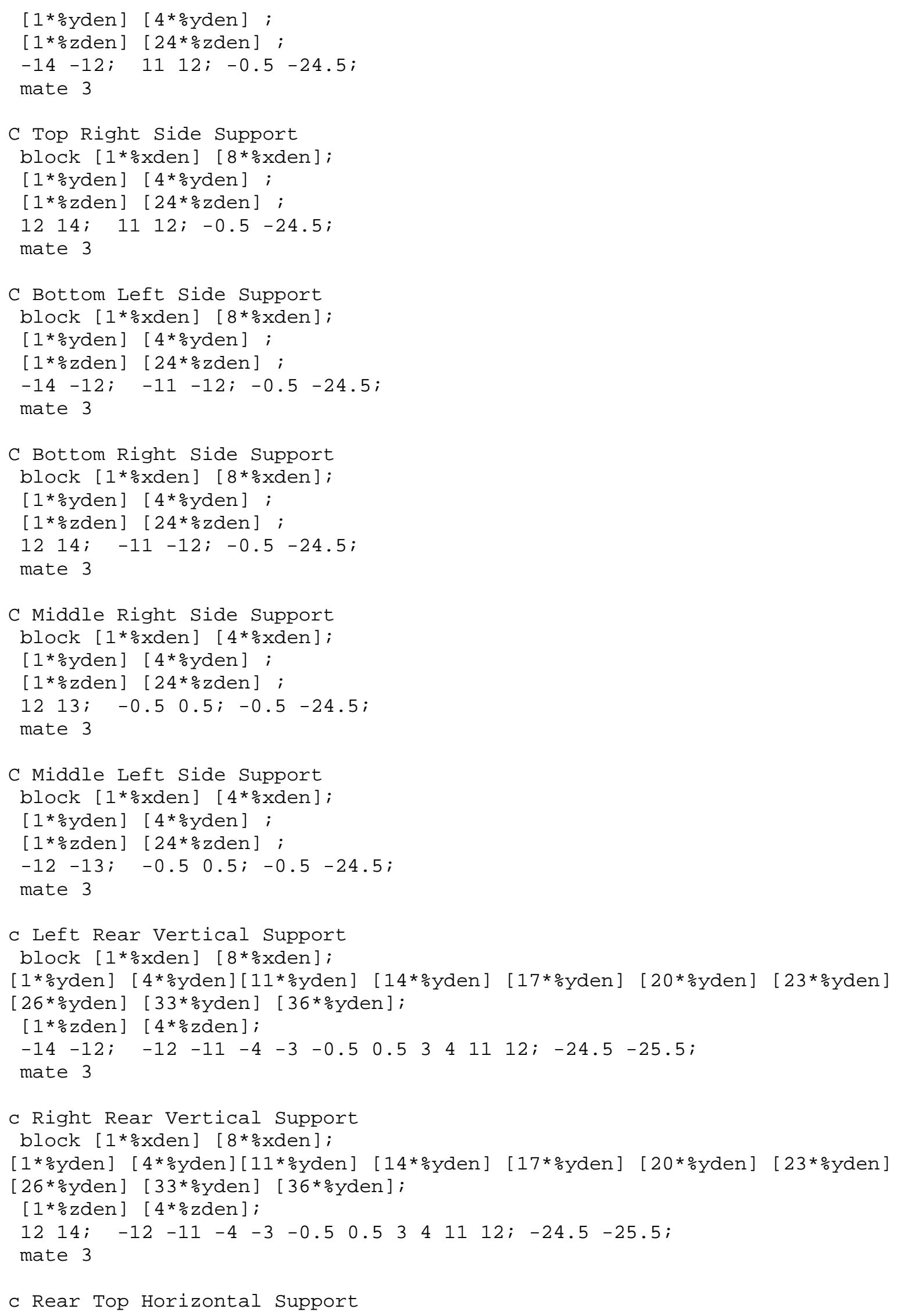




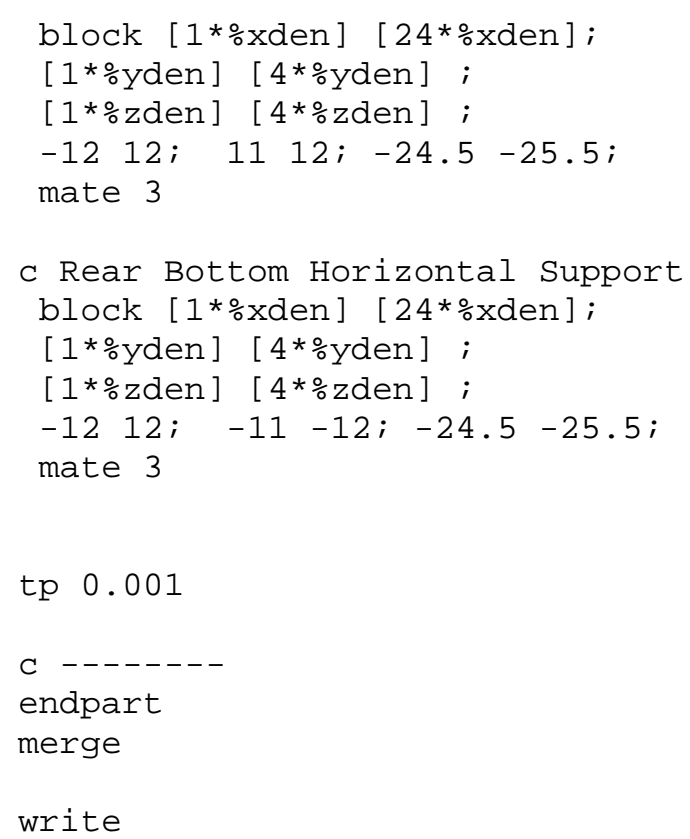




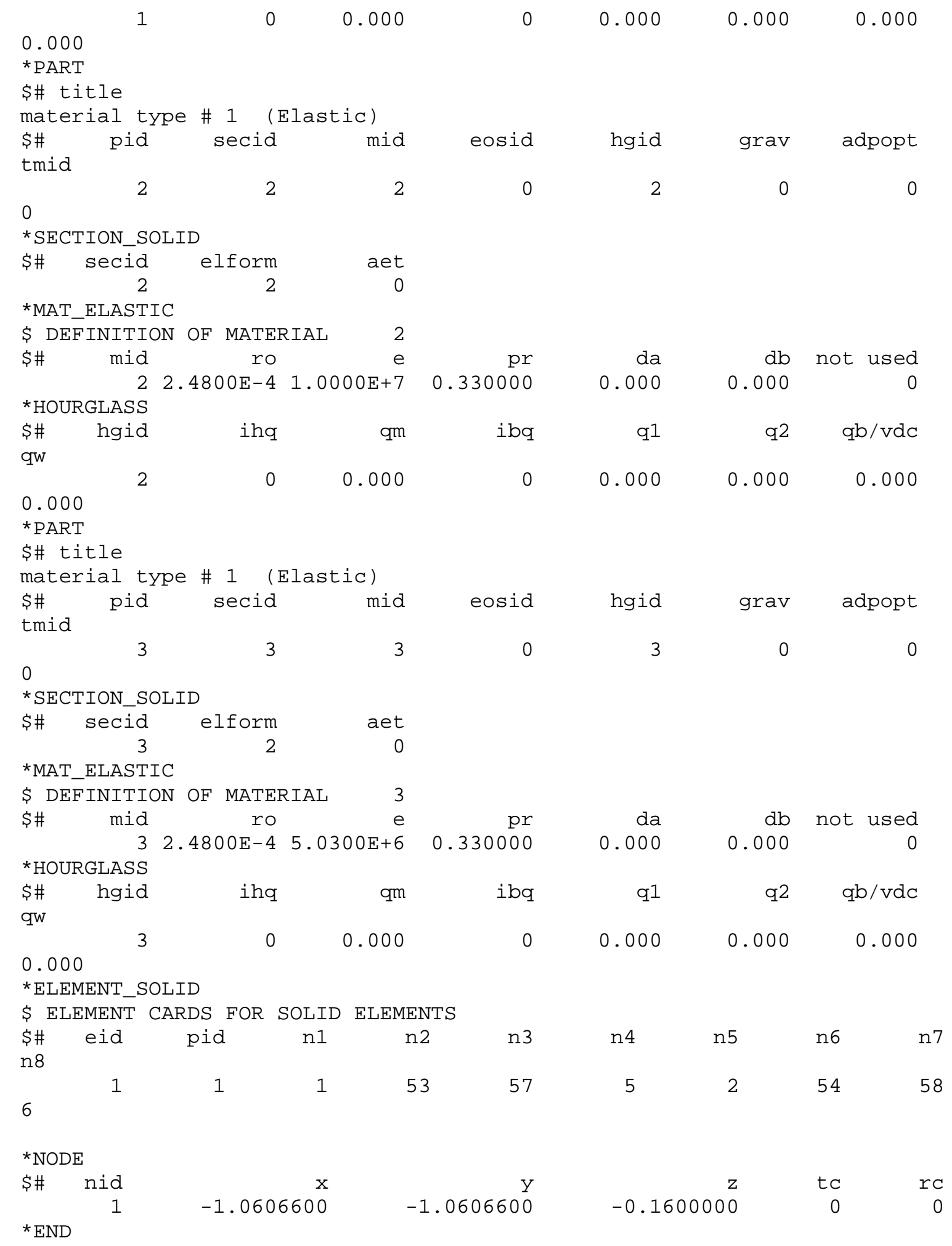


\$\# LS-DYNA Keyword file created by LS-PREPOST 2.4 - 21Jul2009(15:04)

\$\# Created on Dec-02-2009 (22:12:04)

${ }^{*}$ KEYWORD

*TITLE

\$\# title

Titanium Plate Modal

*CONTROL_IMPLICIT_EIGENVALUE

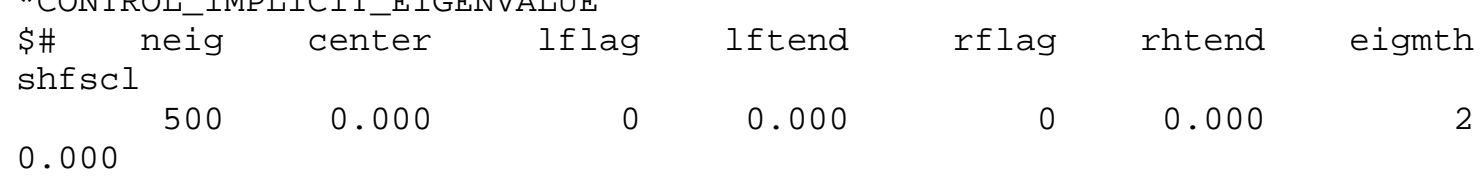

$\$ \#$ isolid ibeam ishell itshell

*CONTROL_IMPLICIT_GENERAL

\$\# imflag dto imform nsbs igs cnstn form

zero_v

$$
1 \quad 0.00 \odot
$$

1

$\odot$

${ }^{*}$ CONTROL_TERMINATION

\$\# endtim endcyc $1.000000 \quad 0$

*BOUNDARY_SPC_SET

$\$ \#$ nsid dof dofy dofy dofry
dofrz

$1 \quad 0$

$\begin{array}{rrrrr}\text { dtmin } & \text { endeng } & \text { endmas } & & \\ 0.00 \odot & 0.00 \odot & 0.00 \odot & & \\ \text { dofx } & \text { dofy } & \text { dofz } & \text { dofrx } & \text { dofry } \\ 1 & 1 & 1 & 1 & 1\end{array}$

1

*SET_NODE_LIST_TITLE

BCS

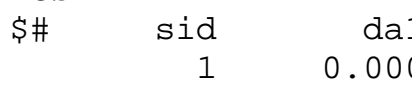

$\$ \#$ nid1 nid2

da2 da3 da4

nid8

$9383 \quad 938$

9408

*PART

$\$ \#$ title

material type \# 1 (Elastic)

\$\# pid secid mid eosid hgid grav adpopt

tmid

1

1

1

1

$\odot$

$\odot$

$\odot$

*SECTION_SOLID

\$\# secid elform aet

*MAT_ELASTIC

\$ MATERIAL CARDS

\$ DEFINITION OF MATERIAL 1

$\$ \#$ mid ro

1 4.1400E-4 1.4500E+7

*HOURGLASS

$\$ \#$ hgid

ihq $\quad$ qm

pr

$\odot .000$

0.000

nid5

nid6

nid7

$\begin{array}{lll}9396 & 9397 & 9407\end{array}$

qW

1

$\odot \quad 0.00 \odot$

$\odot \quad 0.0 \odot \odot$

0.340000

da

$\mathrm{db}$ not used

$\odot .00 \odot$

ibq

q1

q2 qb/vdc

${ }^{*}$ PART

\$\# title

material type \# 1 (Elastic) 


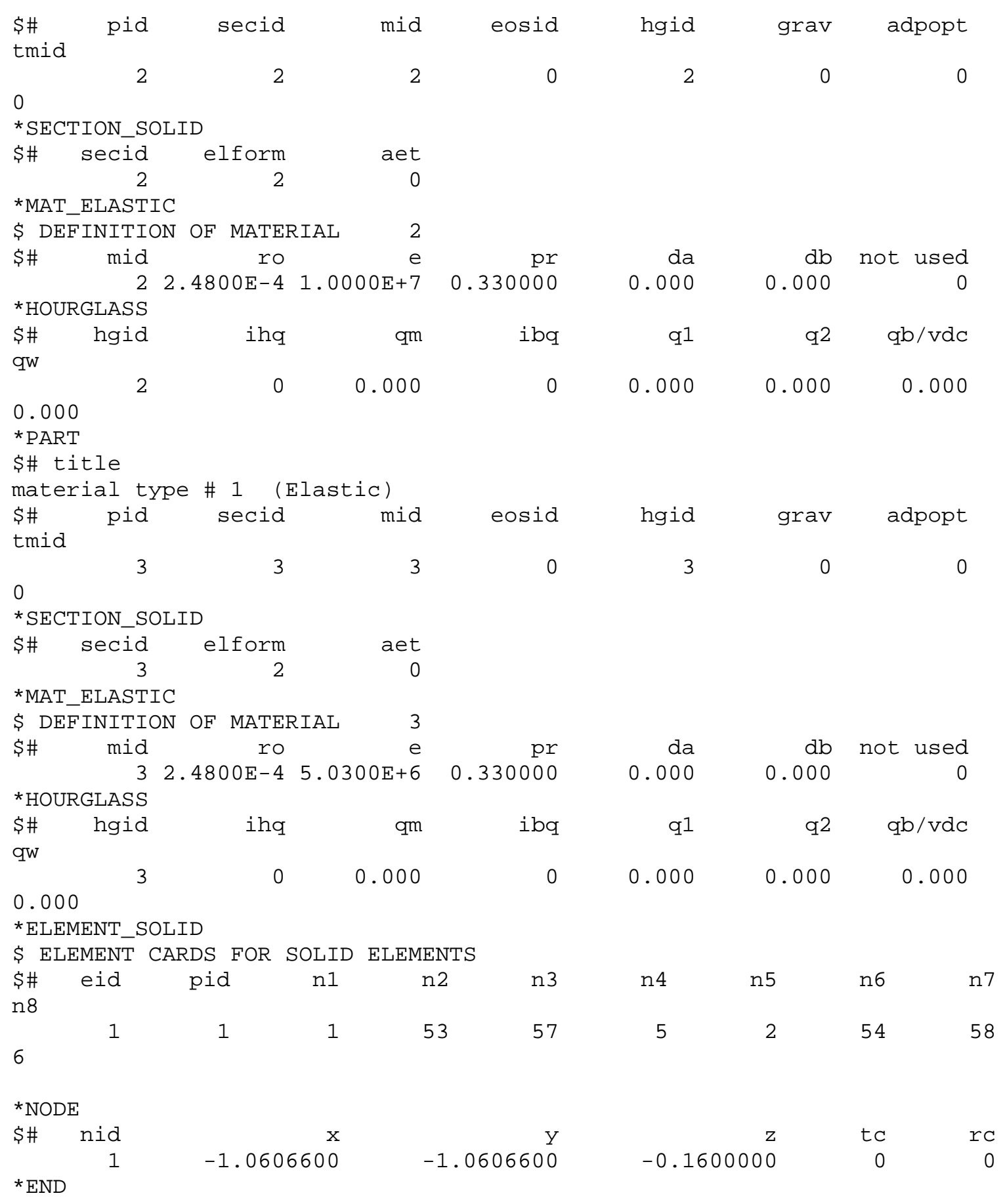


\$\# LS-DYNA Keyword file created by LS-PREPOST 2.4 - 21Jul2009(15:04)

\$\# Created on 0ct-07-2009 (15:59:36)

${ }^{*}$ KEYWORD

*TITLE

\$\# title

Acoustic Simulation of Driver Head

*PARAMETER

$R$ face

$\odot .030000$

$\mathrm{R}$ end

$\odot .108260 \mathrm{R}$ toe

$0.030000 \mathrm{R}$ heel

$\odot .030000 \mathrm{R}$ hozzle

$\odot .090000$

${ }^{*}$ CONTROL_TERMINATION

\$\# endtim endcyc $0.25 \quad 0$

${ }^{*}$ CONTROL_TIMESTEP

$\$ \#$ dtinit tssfac

ms1st

$0.000 \quad 0.900000$

$\begin{array}{rrr}\text { dtmin } & \text { endeng } & \text { endmas } \\ \odot . \odot \odot \odot & \odot . \odot \odot \odot & \odot . \odot \odot \odot\end{array}$

$\odot$

\$\# dt2msf dt2mslc imscl

$0.00 \odot$
*DATABASE_NODOUT

$\$ \#$ dt binary

1. $\odot \odot \odot \odot E-5$ binary lcur

*DATABASE_RBDOUT

\$\# dt binary lcur ioopt

$\begin{array}{llll}1.0000 \mathrm{-}-5 & 0 & 0 & 1\end{array}$

*DATABASE_SECFORC

\$\# dt binary lcur ioopt

$\begin{array}{cccc}2.0000 \mathrm{-} 6 & 0 & 0 & 1\end{array}$

*DATABASE_BINARY_D3PLOT

$\$ \#$ dt lcdt beam npltc psetid

\$\# $\begin{array}{r}0.10000 \\ \text { ioopt } \\ 0\end{array}$

*BEM_ACOUSTIC

$\$ \#$ ro $C$ fmin fmax nfreq dt_out $t$ _start

pref

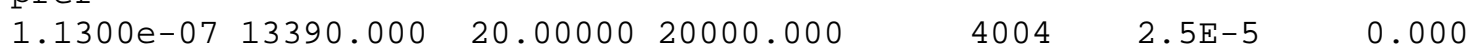

2. $90 \mathrm{e}-09$

\$\#nsid_ext

400

type_ext

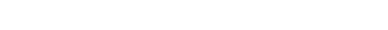

fft win

$$
\text { trslt ipfile }
$$

\$\# method $\operatorname{maxit}$

$10001.0000 \mathrm{E}-6$

\$\# ssid

sstype

norm bem_type

ndd

1

11

*DATABASE_CROSS_SECTION_SET_ID

$\$ \# \quad \operatorname{csid}$

title

$\$ \#$ nsid

itype

$\odot$

221

hsid

bsid

ssid

tsid

dsid

id

*SET_NODE_LIST

$\$ \$$

\$\$ Sets Defined In HyperMesh

$\odot \quad 222$

$\odot$

$\odot$

$\odot$ 
$\$ \$$

\$HMSET

\$HMNAME SETS 1crown-face section nodes

\$\# sid da1 da2 da3

$\begin{array}{lrrrrrrr} & 221 & 0.00 \odot & 0.00 \odot & 0.00 \odot & 0.00 \odot & & \\ \text { \$\# } & \text { nid1 } & \text { nid2 } & \text { nid3 } & \text { nid4 } & \text { nid5 } & \text { nid6 } & \text { nid7 }\end{array}$

100050

100043

100044

100045

100046

$100047 \quad 100048$

100049 100051

$100052 \quad 100053$

100054

$100055 \quad 100056$

100057

100058

100059

100060

100061

$10 \odot \odot 62$

$100063 \quad 100064$

100065

100066

100067

$10 \odot \odot 68$

100069

100070

$100071 \quad 100072$

100073

100486

*DATABASE_HISTORY_NODE

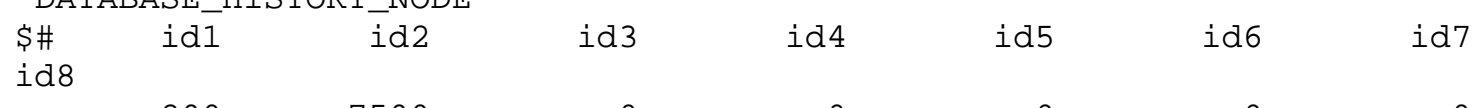

${ }^{*}$ BOUNDARY_SPC_SET
\$

$\$ \#$ nsid dofx dofy dofz dofrx dofry dofrz

$401 \quad 0$

1

1

1

1

1

1

*SET_NODE_LIST_TITLE

Hozzle

$\$ \#$ sid da1

$401 \quad 0.000$

$\$ \#$ nid1 nid2

da2

0.000

da3

0.000

da4

nid3

nid4

0.000

nid5

nid6

nid7

nid8

50005

60004

60004

600002

50009

50008

50006

50007

50004

$50003 \quad 500 \odot 2$

50001

$600 \odot 46$

600034

50012

${ }^{\star}$ CONTACT_SURFACE_TO_SURFACE_ID

$\$ \#$ cid

title

0

\$\# ssid

mpr

msid

sstyp

mstyp

sboxid

mboxid

spr

2

3

0

$\odot$

$\odot$

$\odot$

\$\#

fs fd

dc

vc

vdc penchk

bt

0.300000

0.300000

$\odot .00 \odot$

$\odot .000$

$\odot .0 \odot \odot$

0

0.000

0.000

\$\# sfs

sfm

sst

mst

sfst

sfmt

fsf

1.000000

0.000

$\odot .10 \odot \odot \odot \odot$

$\odot .00 \odot$

0.000

$\odot .000$

0.000

0.000

$\$ \#$ soft sofscl

lcidab

maxpar

sbopt

depth

bsort

frcfro

$\odot \quad 0.00 \odot$

$\odot$

0.000

$\odot .000$

$\odot$

$\odot$

0

*SET_PART_LIST

$\$ \#$ sid

da1

da2

da3

da4 


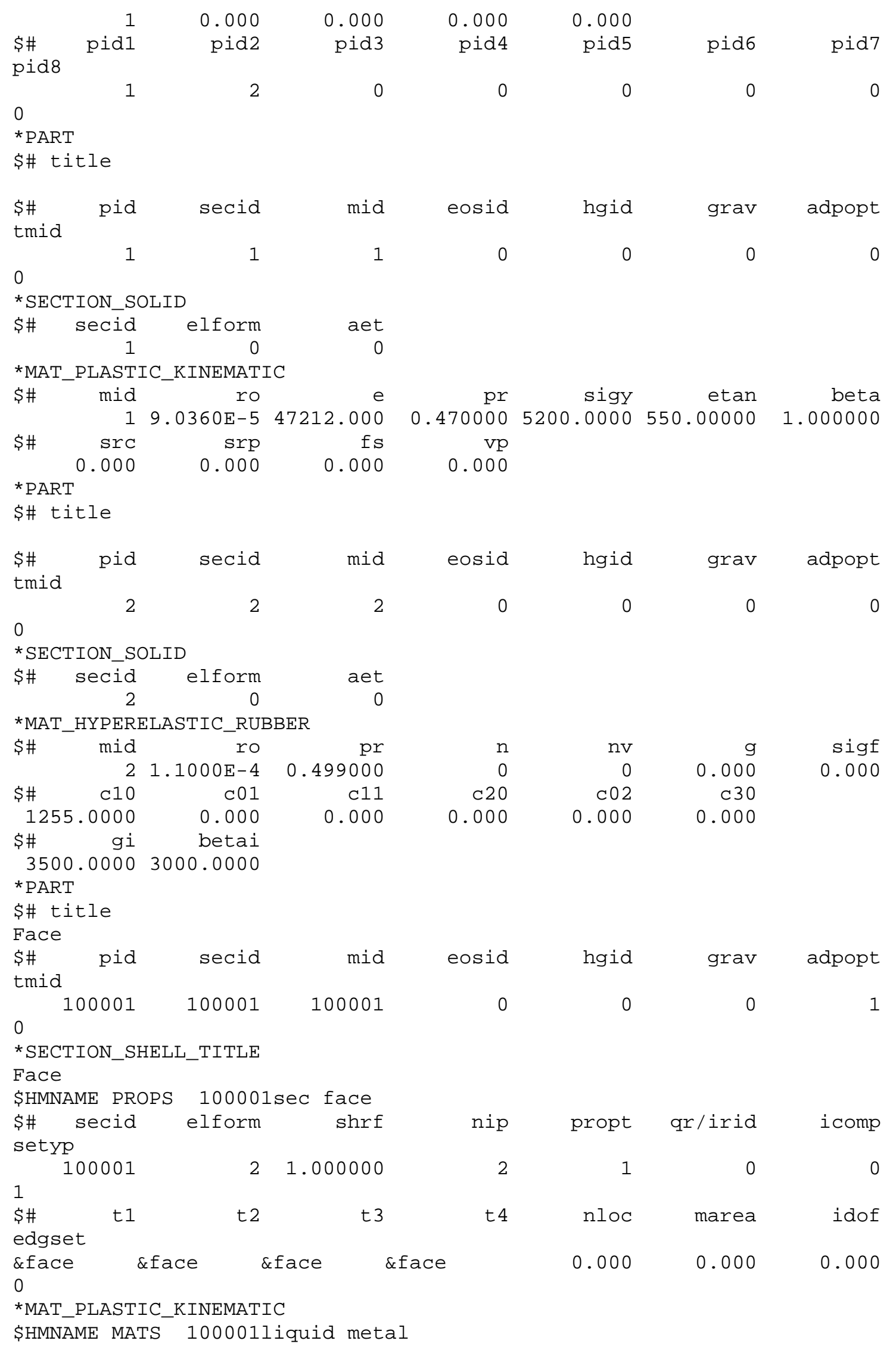




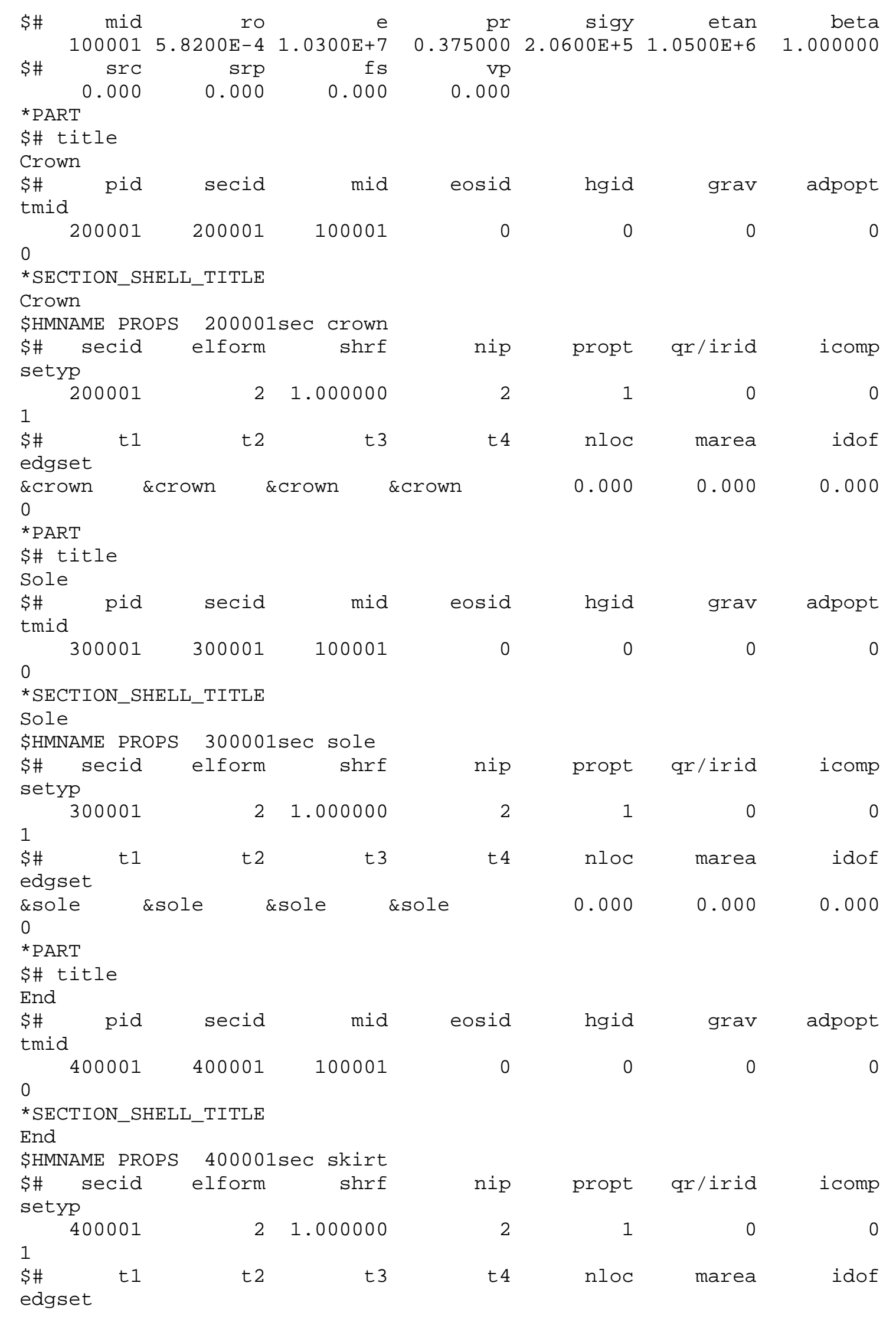




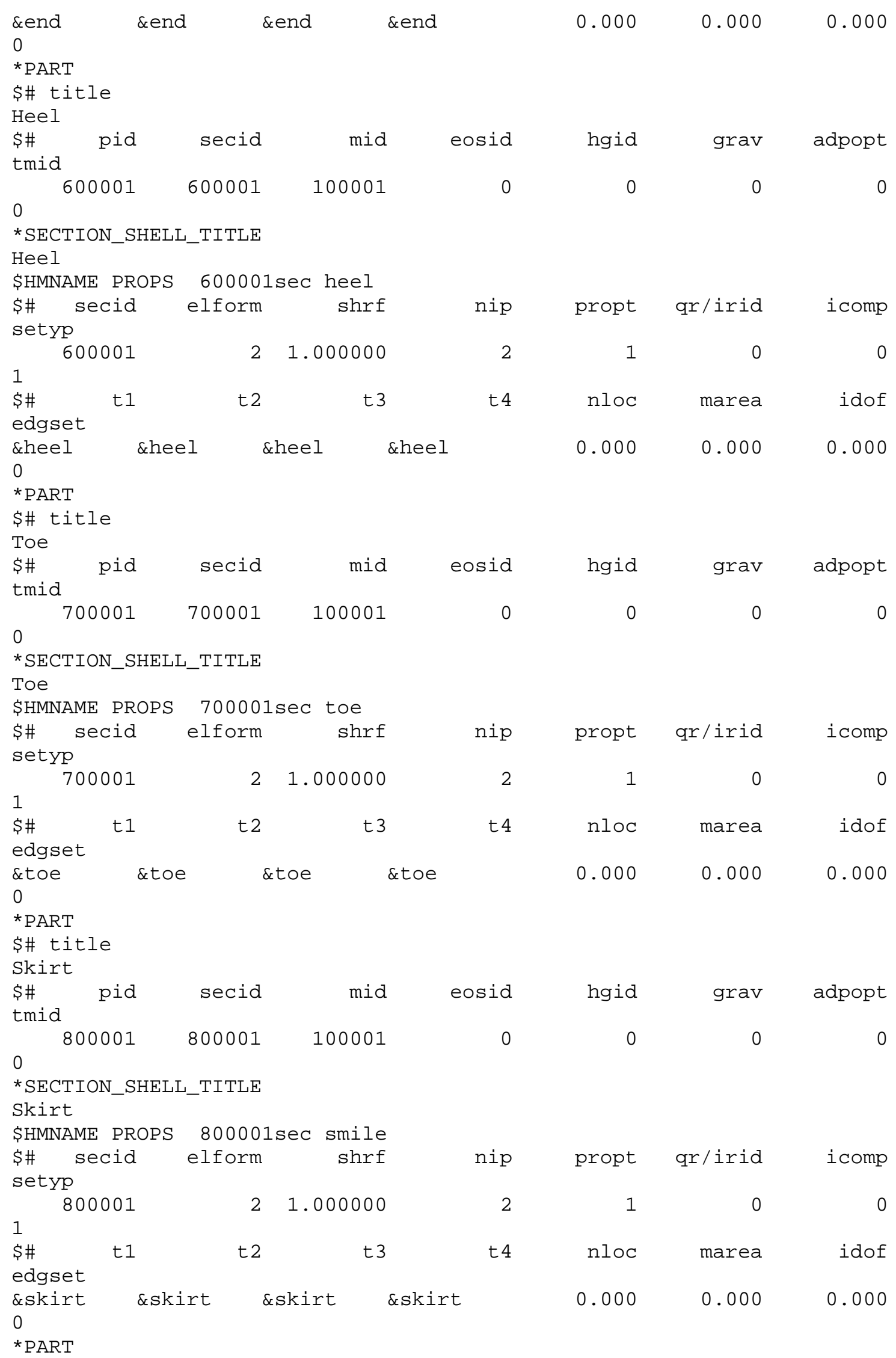




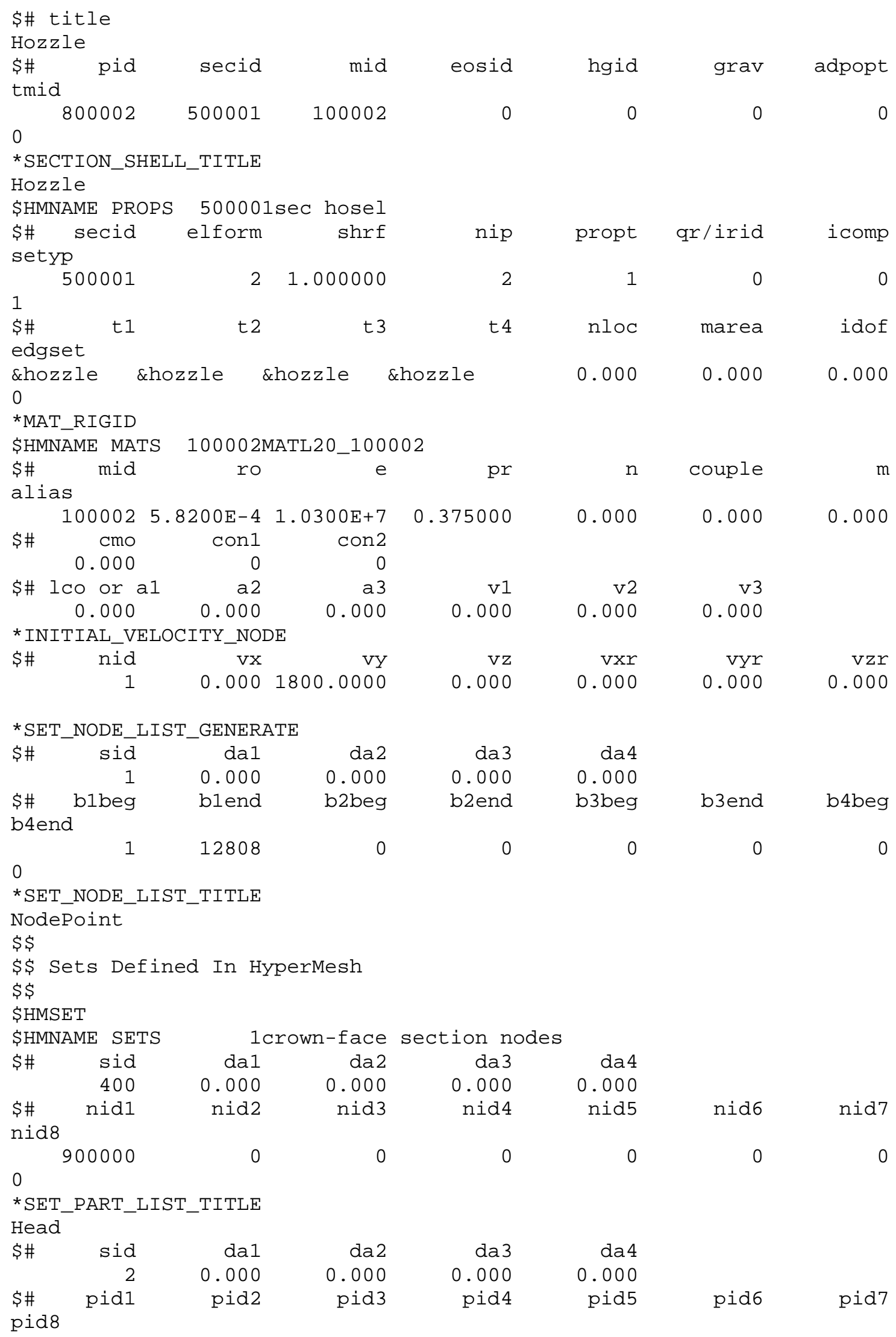




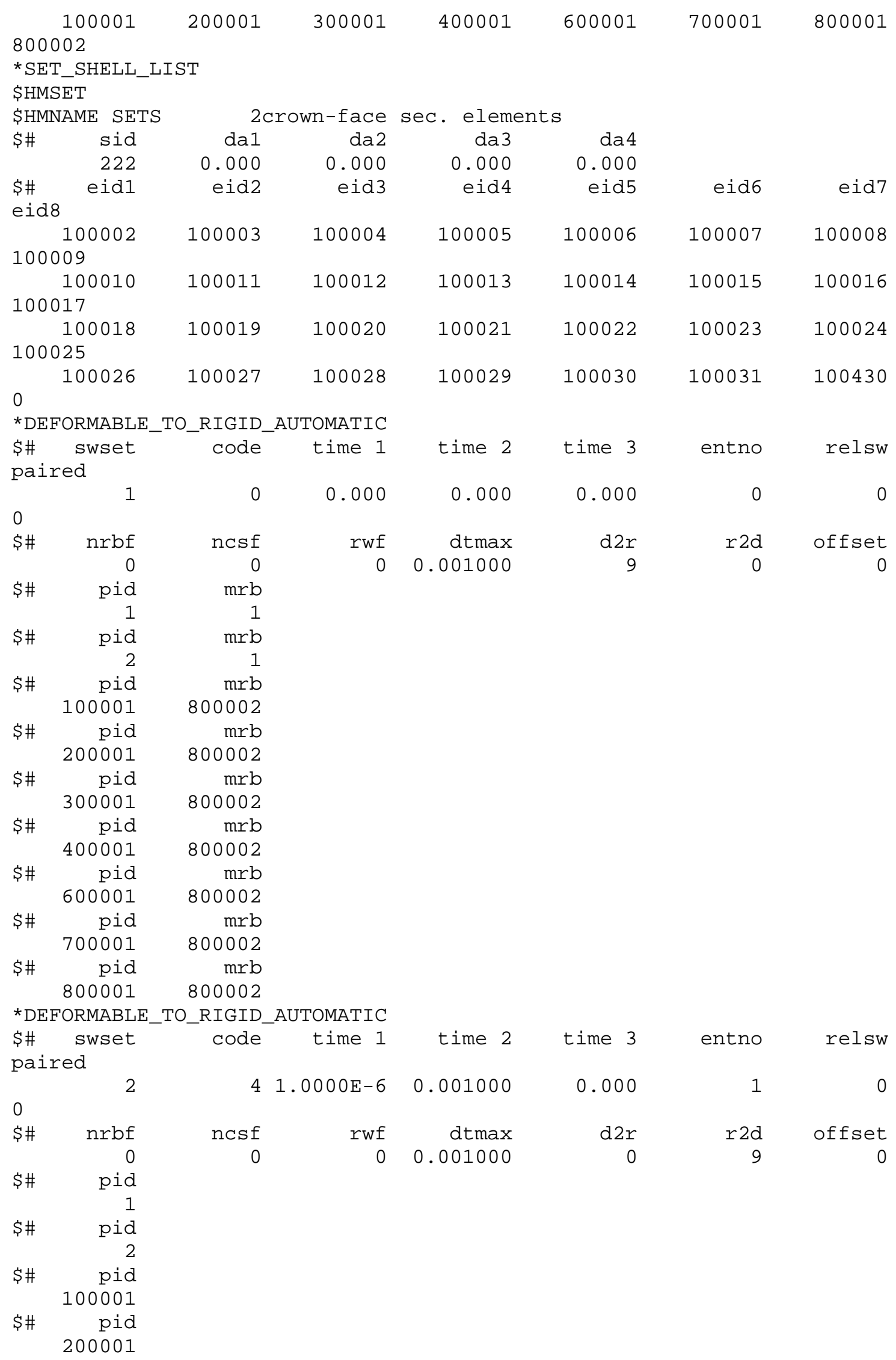




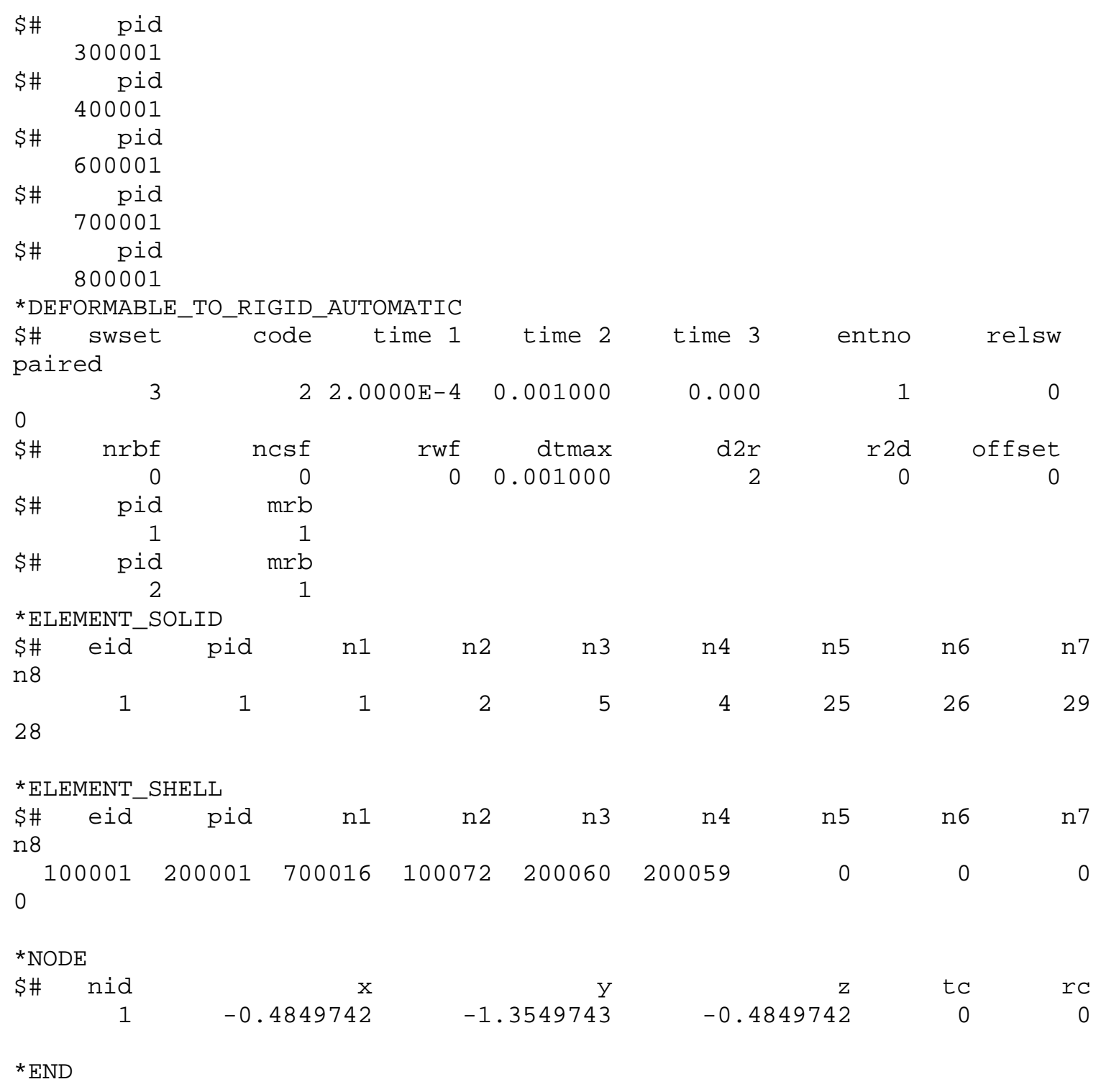




\section{Appendix D: MATLAB ${ }^{\text {TM }}$ Files}

$\%$ Output data from clubhead impacts . wav files provided

\% Roger Sharpe

clear all

close all

clc

disp('Which club would you like to look at?')

disp('1 for Cobra')

disp('2 for Ping')

disp(' 3 for Cleveland')

flag = input ('Your choice: ');

if $\mathrm{flag}==1$

input_name $=$ 'Cobra LD Impact';

elseif flag $==2$

input_name = 'Ping G5 Impact';

elseif flag $==3$

input_name = 'Launcher DST Impact';

else

end

disp('incorrect value')

$[y$, Fs, bits $]=$ wavread $($ input_name $) ;$

clc

disp('Want to hear the file? (1 for Y/ 2 for N)')

soundflag = input ('Your choice: ');

if soundflag $==1$

wavplay $(\mathrm{y}, \mathrm{Fs})$;

elseif soundflag $==2$

else

disp('Skipping sound...')

end

disp('Something went wrong')

amp $=y(:, 1) ;$

$\mathrm{spl}=\operatorname{amp}(1: 11025)$;

$\mathrm{z}=\mathrm{fft}(\mathrm{spl})$;

$\mathrm{N}=$ length $(\mathrm{z})$;

$\mathrm{T}=0.25 ; \quad \%$ secs

$\mathrm{df}=1 / \mathrm{T}$;

Fsamp $=N^{*} d f$;

$f=d f^{*}(0: N-1)$;

time $=\operatorname{linspace}(0,0.25,11025)$;

figure(1) 


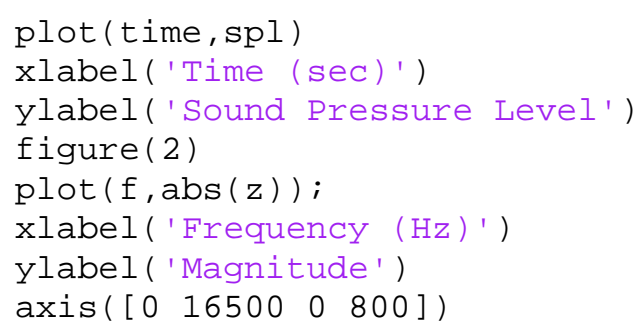




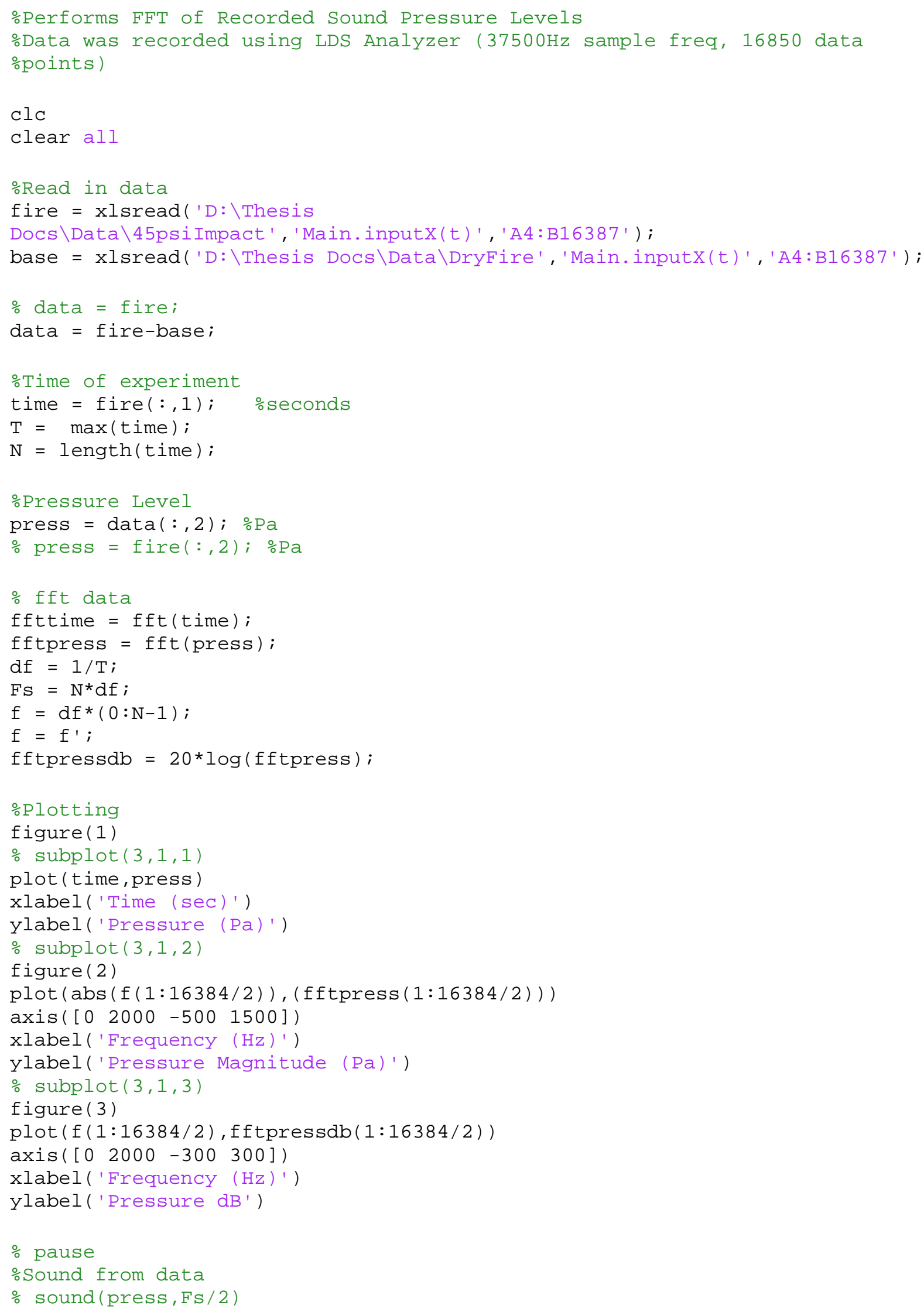




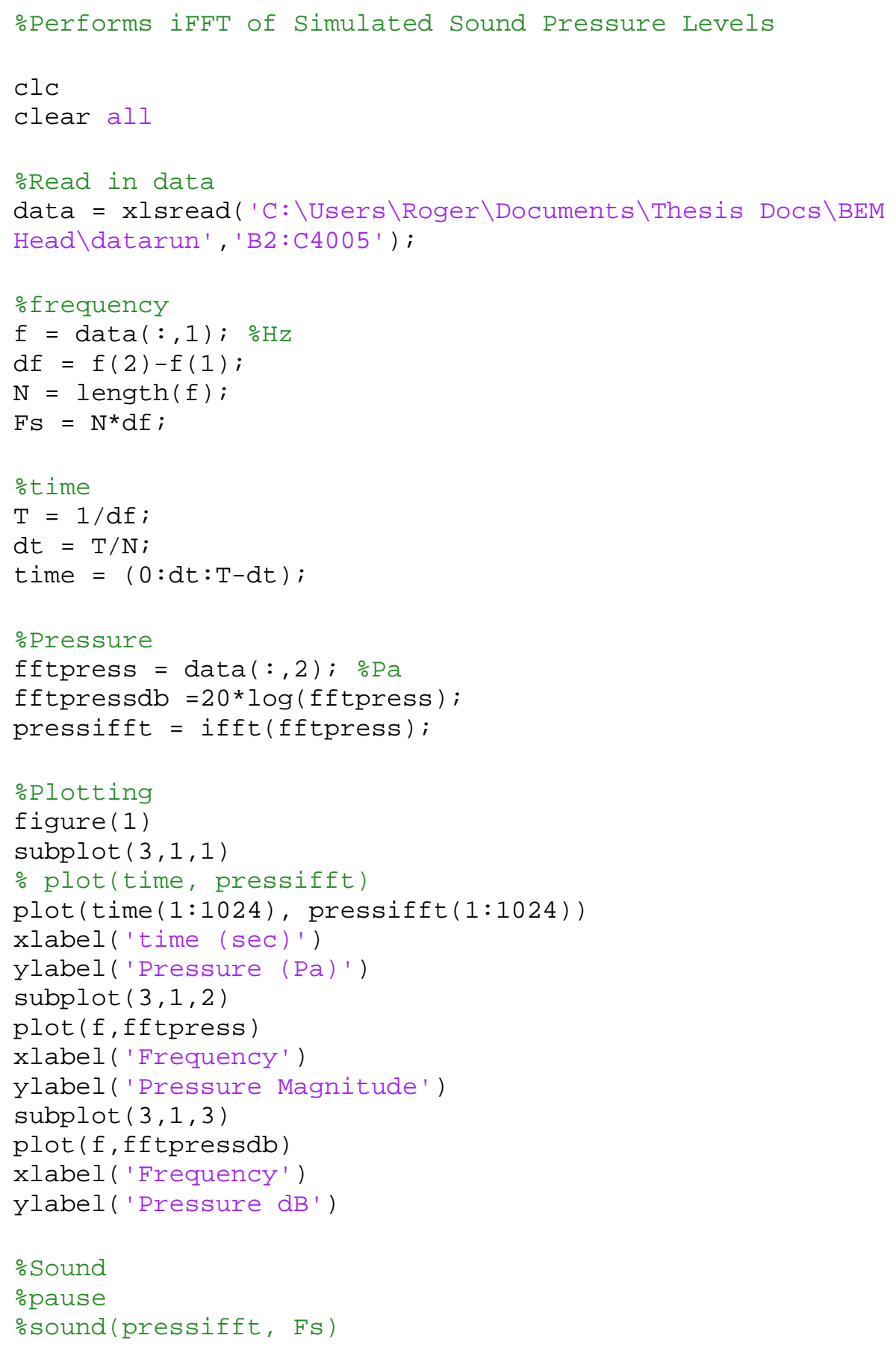

